$$
\begin{gathered}
B 2505=759 \\
Q C \\
966.7 \\
.05 \\
624 \\
5005
\end{gathered}
$$

\title{
A PERFORMANCE ANALYSIS OF THE NORTH AMERICAN LIGHTNING DETECTION NETWORK USING CN TOWER LIGHTNING DATA
}

\author{
Alexandru Lafkovici \\ B.Eng., Ryerson University, Toronto, 2003
}

A thesis

presented to Ryerson University

in partial fulfillment of the

requirements for the degree of

Master of Applied Science in the Program of

Electrical and Computer Engineering.

Toronto, Ontario, Canada, 2005

(C)Alexandru Lafkovici 2005 


\section{Author's Declaration}

I hereby declare that I am the sole author of this thesis.

I authorize Ryerson University to lend this thesis to other institutions or individuals for the purpose of scholarly research.

I further authorize Ryerson University to reproduce this thesis by photocopying or by other means, in total or in part, at the request of other institutions or individuals for the purpose of scholarly research. 


\section{Instructions for Borrowers}

Ryerson University requires the signatures of all persons using or photocopying this thesis. Please sign below, and give address and date.

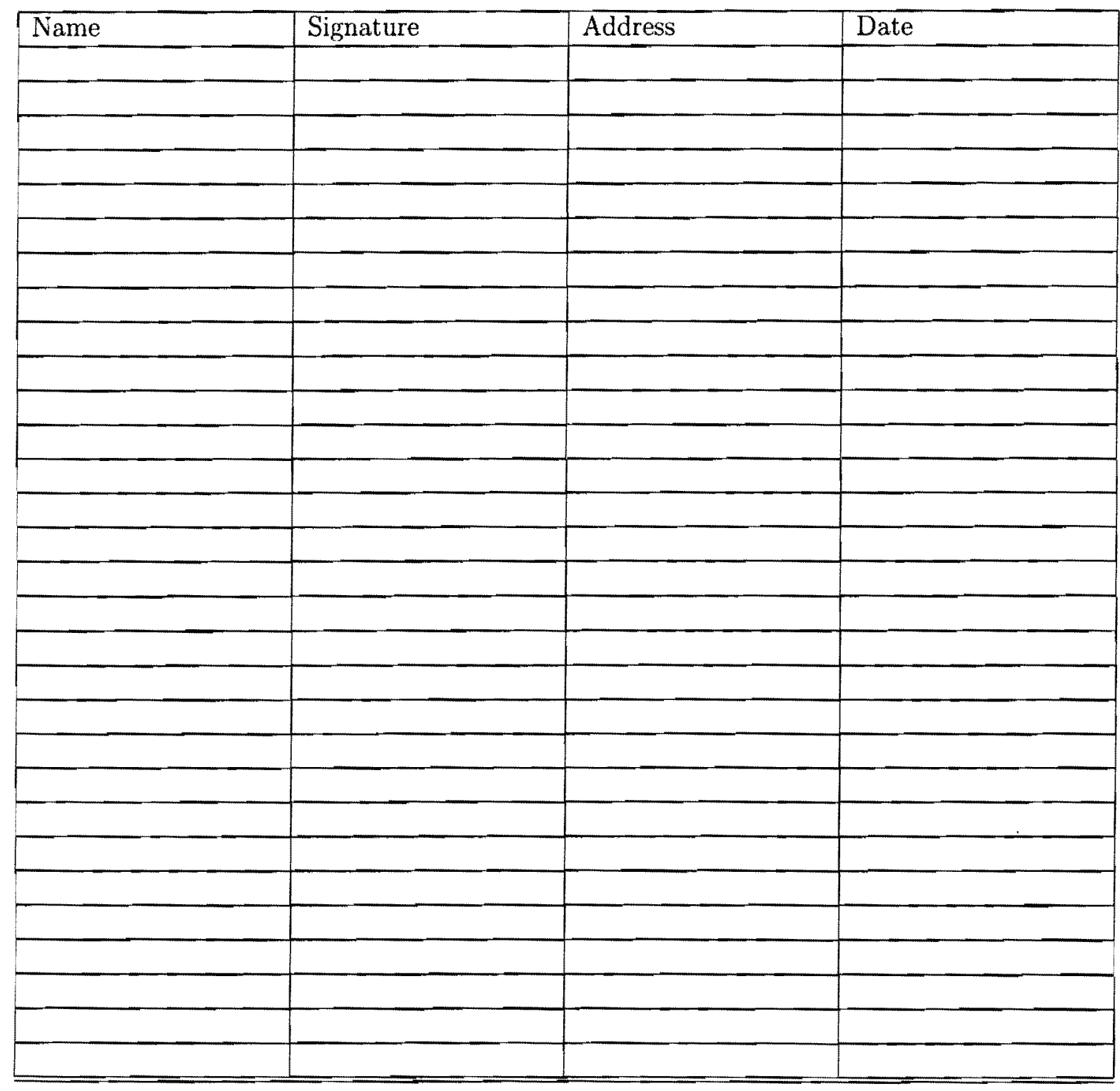




\title{
Abstract \\ A Performance Analysis of the North American Lightning Detection Network using CN Tower Lightning Data
}

\author{
(C)Alexandru Lafkovici 2005 \\ Master of Applied Science \\ Department of Electrical and Computer Engineering \\ Ryerson University
}

The North American Lightning Detection Network (NALDN) is a commercial lightning detection network operated by Vaisala Inc., and is composed of the U.S. National Lightning Detection Network (NLDN) and the Environment Canada owned Canadian Lightning Detection Network (CLDN). The CN Tower is one of the best sites in the world to observe the lightning phenomenon and provides an excellent opportunity to evaluate the performance of the NALDN in the Toronto area. Using CN Tower lightning data acquired during 2005, the performance characteristics of the NALDN were thoroughly evaluated, including the flash detection efficiency (DE), stroke $\mathrm{DE}$, absolute location error, peak current estimation and location accuracy model (50\%, $90 \%$ and $99 \%$ error ellipses) error. Although a similar test was performed using rocket-triggered lightning in Florida at Camp Blanding, this test evaluated a completely different region of the NALDN. Moreover, rocket-triggered lightning artificially initiates a lightning discharge, whereas lightning events to the CN Tower occur naturally and are similar to discharges that occur to tall structures or objects at high altitude or mountainous areas.

Excluding two flashes understood to be composed of M-components, the NALDN detected 7 out of 7 flashes recorded at the CN Tower, resulting in a $100 \%$ percent flash DE. Furthermore, the NALDN detected 22 out of 39 strokes recorded at the CN Tower, resulting in a stroke DE of $56 \%$. Relative to the CN Tower, the NALDN was found to have a median absolute location error of $0.356 \mathrm{~km}$ and a mean error of $0.390 \mathrm{~km}$ for the 22 strokes it detected. It was also demonstrated that the NALDN stroke location error seems to have a large bias towards the north of the CN Tower and a slight bias towards the east, with 19 out of the 22 strokes predicted north-east of the CN Tower.

The $50 \%, 90 \%$ and $99 \%$ error ellipses provided by the NALDN were also evaluated. It was found that $73 \%$ ( 16 out of the 22 ) detected strokes were enclosed by the $50 \%$ error ellipse, $91 \%$ ( 20 out of the 22 ) detected strokes were enclosed by the $90 \%$ error ellipse and $95 \%$ (21 out of the 22) detected strokes were enclosed by the $99 \%$ error ellipse. The minimum value for the $50 \%$ error ellipse axes is set at $0.4 \mathrm{~km}$ by Vaisala, and 21 out of the 22 detected strokes had a semi-major axis length of $0.4 \mathrm{~km}$, suggesting that the median location error for CN Tower strokes is $0.4 \mathrm{~km}$ or less. The $0.356 \mathrm{~km}$ median location error obtained for the 22 detected strokes appears to support this. 


\section{Acknowledgments}

I would like to thank my family, particularly my mother Angela Lafkovici and my father Vladimir Lafkovici for all their support during my studies in electrical engineering. I would also like to thank Sri Vasudeva for his spiritual support and guidance.

I want to thank my thesis supervisor Dr. Ali M. Hussein for his support, insight and guidance on this project. It was an extremely interesting experience on what is undoubtedly a fascinating research project.

I am very grateful to the Ontario Government for the Ontario Graduate Scholarship and the Ontario Graduate Scholarship in Science and Technology that I was consecutively awarded for my two years of study.

I would like to express my gratitude to Professor Wasyl Janischewskyj for providing me with the opportunity to conduct this research. I want to thank the whole CN Tower lightning team that I worked with for their assistance and support, particularly Saad Faisal, Davide Pavanello, Mariusz Milewski and Faizan Jabbar.

I would also like to thank Chief Scientist Ken Cummins and Research Engineer John Cramer of Vaisala Inc. for providing me with North American Lightning Detection Network data at the critical hour, and for generously taking the time to answer questions and offer comments and advice. I also want to offer my appreciation to Kelsey Spring from Environment Canada for granting us permission to use this data for the purpose of this research. 


\section{Contents}

List of Tables $\quad$ viii

List of Figures $\quad$ ix

1 Introduction $\quad 1$

2 Lightning Location Basics $\quad 5$

2.1 Lightning Emissions . . . . . . . . . . . . . . . . . . . . 6

2.2 Influences on Electromagnetic Wave Propagation through the Atmosphere . 8

2.3 Lightning Detection Methods used in the North American Lightning Detection

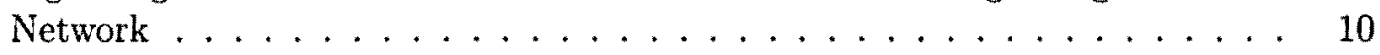

2.3.1 Magnetic Field Direction Finding ............... 11

2.3 .2 Time-of-arrival technique . . . . . . . . . . . . . 16

2.4 Summary . . . . . . . . . . . . . . . . . . . . . 19

3 The North American Lightning Detection Network 21

3.1 History . . . . . . . . . . . . . . . . . . . . . . . . 22

3.2 Development and Sensor Locations . . . . . . . . . . . . . . . . . 25

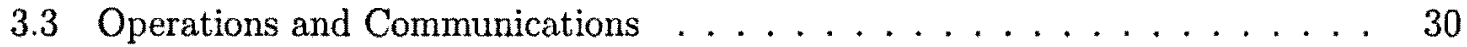

3.4 Location and Stroke Processing Algorithms . . . . . . . . . . . . . . . . . . . . . . . . . . . . . . . . .

3.4 .1 The IMPACT Location Method . . . . . . . . . . . . . . . . . 35

3.4 .2 Flash Multiplicity and Polarity ............... 39

3.4 .3 Flash and Stroke Times .................. 41

3.4.4 Peak Current Estimation . . . . . . . . . . . . . . . . 41

3.5 Measures of Performance: Location Accuracy and Detection Efficiency . . 43

3.5 .1 Location Accuracy Model . . . . . . . . . . . . . . . . . . . . . 43

3.5 .2 Detection Efficiency Model . . . . . . . . . . . . . . . . 46

3.6 Comparison of Past and Present Performance . . . . . . . . . . . . . . 47

3.6 .1 Location Accuracy ... . . . . . . . . . . . . . . . . . . . . . . . . . . . . .

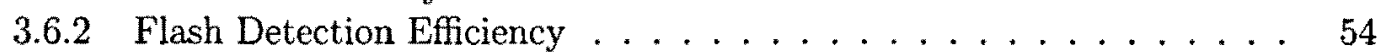

3.6 .3 Stroke Detection Efficiency . . . . . . . . . . . . . . 58

3.6 .4 Peak Current . . . . . . . . . . . . . . . . . . . 61

3.6 .5 Misclassified Events . . . . . . . . . . . . . . . . . 63

3.6 .6 Summary . . . . . . . . . . . . . . . . . . 64 
3.7 Applications . . . . . . . . . . . . . . . . . . . . 66

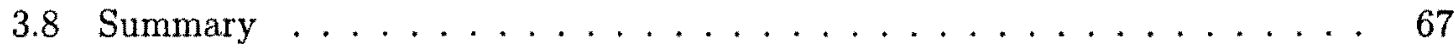

4 North American Lightning Detection Network Performance Analysis using CN Tower lightning data $\quad \mathbf{7 0}$

4.1 The CN Tower Lightning Project $\ldots \ldots \ldots \ldots \ldots \ldots \ldots$

4.1 .1 Overview . . . . . . . . . . . . . . . 71

4.1.2 Current Derivative Measurement System . . . . . . . . . . . 73

4.1 .3 Field Measurement System . . . . . . . . . . . . . . . . . 74

4.1 .4 Summary . . . . . . . . . . . . . . . . 76

4.2 North American Lightning Detection Network

Performance Analysis . . . . . . . . . . . . . . . 78

4.2 .1 Detection Efficiency . . . . . . . . . . . . . . . . . . . 89

4.2 .2 Location Accuracy . . . . . . . . . . . . . . . . . . . 93

4.2.3 Peak Current Estimation . . . . . . . . . . . . . . . . 107

4.3 Discussion . . . . . . . . . . . . . . . . . . . . . . 109

4.3.1 Detection Efficiency . . . . . . . . . . . . . . . . . . . . 109

4.3 .2 Location Accuracy . . . . . . . . . . . . . . . . . . . 112

4.3.3 Peak Current Estimation . . . . . . . . . . . . . . . 117

4.3.4 Comparison to the Findings from other Evaluations . . . . . . . 118

4.4 Summary . . . . . . . . . . . . . . . . . . . 120

5 Conclusions and Recommendations 122

5.1 Conclusions . . . . . . . . . . . . . . . . . . . . . . 122

5.2 Recommendations . . . . . . . . . . . . . . . . . . . 125

$\begin{array}{ll}\text { Bibliography } & 128\end{array}$

$\begin{array}{ll}\text { Appendix } & 133\end{array}$

$\begin{array}{ll}\text { A Abbreviations } & 133\end{array}$ 


\section{List of Tables}

3.6.1 Summary of the NALDN projected performance. (Adapted from [24].) _. 65

4.2.1 NALDN data Analysis using CN Tower lightning data from 2005. . . . . 80

4.2.2 Summary of the total lightning events recorded at the CN Tower, along with the corresponding NALDN detection efficiencies and absolute location errors.

4.2.3 Summary of the flashes and strokes recorded at the CN Tower, excluding the events thought to be M-components, along with the corresponding NALDN detection efficiencies and absolute location errors. . . . . . . . . . .

4.2.4 NALDN sensor configuration in the region around the CN Tower, including the distance of each sensor from the CN Tower. The sensor location information was provided by Vaisala $[51] . \ldots \ldots \ldots \ldots \ldots \ldots$

4.2.5 The mean and median North-South and East-West NALDN location errors relative to the $\mathrm{CN}$ Tower, along with the corresponding standard deviations. 


\section{List of Figures}

2.1.1 Relationship between frequency and lightning, along with the various VLF, LF, and VHF lightning detection technologies. (Adapted from [10].) . . . .

2.1.2 Electromagnetic radiation generated by the different physical processes in lightning. (Adapted from [11].) ............... 8

2.3.1 Components of a LLP magnetic DF station used in the Ontario provincial LLS in 1989. (Adapted from [13].) . . . . . . . . . . . . . . . .

2.3.2 Determination of a lighting stroke location using only two DFs. (Adapted

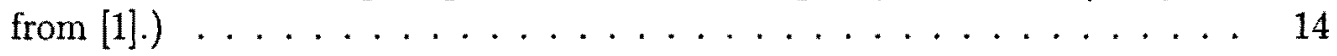

2.3.3 Baseline problem that may occur when using only two DFs to detect a stroke. (Adapted from $[1]$. ) . . . . . . . . . . . . . .

2.3.4 Determination of a lighting stroke location using three DFs. (Adapted from

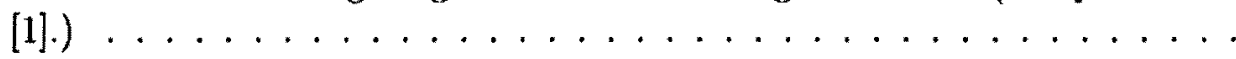

2.3.5 Determination of a lighting stroke location using three TOA sensors when the solution is not unique. (Adapted from [1].) . . . . . . . . . . .

2.3.6 Determination of a lighting stroke location using three TOA sensors when the solution is unique. (Adapted from [1].) . . . . . . . . . . 18

3.2.1 Locations of the sensors in the U.S. NLDN after the 1995 upgrade. (Adapted

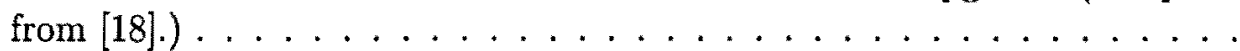

3.2.2 Location of the LLP magnetic DFs in the Ontario LLS in 1989. (Adapted from [13].) . . . . . . . . . . . . . . . . . . .

3.2.3 The NALDN after the 2003 NLDN upgrade. The projected flash detection efficiency is displayed. (Adapted from [5].) . . . . . . . . . . . . . . . . 30

3.3.1 Real-time data flow in the NALDN $[18] \ldots \ldots . \ldots . \ldots . \ldots 32$

3.4.1 An event occurring on the baseline between two IMPACT sensors (Adapted

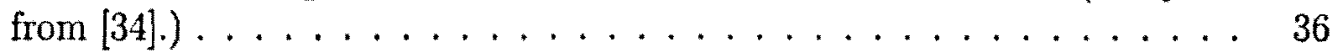

3.4.2 Example of the IMPACT algorithm using three LPATS TOA sensors and two IMPACT sensors. (Adapted from $[10]$. . . . . . . . . . . . .

3.4.3 Angle-based flash grouping algorithm, illustrating how multiplicity can be overestimated. (Adapted from [18].) . . . . . . . . . . . . .

3.4.4 Location-based flash grouping algorithm. Strokes 1, 3, and 4 constitute one flash; stroke 2 is regarded as a separate flash. (Adapted from [18].) . . . . 
3.5.1 Two-dimensional Gaussian distribution of location errors showing the estimated stroke location at the most probable point (the peak). The $50 \%$ error ellipse is derived by cutting the distribution at a probability level of 0.5 . (Adapted from [18]). . . . . . . . . . . . . . .

3.5.2 99\% confidence ellipses for all strikes detected within the search area using data provided by the NALDN. (Adapted from [39]). . . . . . . . . . .

3.6.1 Projected semi-major axis of the error ellipse for the NLDN after the 1995 upgrade. Contour labels give the value in kilometers and represent the median (50\% confidence level) location accuracy. (Adapted from [18]). . .

3.6.2 Locations of seven triggered strokes detected by the NLDN relative to the triggering site. (Adapted from [18].) . . . . . . . . . . . . . .

3.6.3 Projected semi-major axis of the error ellipse for the NALDN after the 2003 NLDN upgrade. Contour labels give the value in kilometers and represent the median (50\% confidence level) location accuracy. (Adapted from [5]). .

3.6.4 Plot of NLDN stroke location errors for 95 strokes in 31 flashes triggered during 2001 to 2003 at Camp Blanding. (Adapted from [8].) . . . . . . . .

3.6.5 NLDN absolute location error versus Camp Blanding peak current, for 70 strokes with measured peak currents in 22 flashes triggered during 2001 to 2003. (Adapted from [8].) . . . . . . . . . . . . . . . .

3.6.6 NLDN absolute location error plotted versus the number of reporting NLDN sensors, for 95 strokes in 31 flashes triggered during 2001 to 2003. (Adapted from $[8].) \ldots \ldots \ldots \ldots \ldots \ldots . \ldots \ldots$

3.6.7 Projected flash DE for the NLDN after the 1995 upgrade for negative flashes with peak currents greater than $5 \mathrm{kA}$. Contour labels give the $\mathrm{DE}$ in percent. (Adapted from [18]) . . . . . . . . . . . . . . . .

3.6.8 Projected flash DE for the NALDN after the 2003 NLDN upgrade. Contour labels give the DE in percent.(Adapted from [5].) . . . . . . . . . . .

3.6.9 NLDN stroke detection efficiency plotted as a function of peak current measured at Camp Blanding. The total number of strokes whose currents were measured at Camp Blanding is 122, of which 70 were detected by the NLDN.

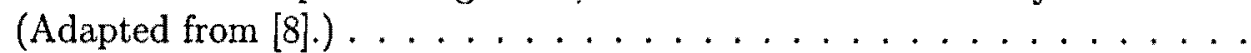

3.6.10 NLDN peak current estimation error (defined as $\Delta I=I_{N L D N}-I_{C B}[$ NLDN estimated peak current minus Camp Blanding measured Peak Current]) plotted versus Camp Blanding peak current, for 70 strokes in 22 flashes triggered during 2001 to 2003. (Adapted from [8].) . . . . . . . . . . .

3.6.11 Number of NLDN reporting sensors plotted versus Camp Blanding peak current, for 70 strokes with measured peak currents in 22 flashes triggered during 2001 to 2003. (Adapted from [8].) . . . . . . . . . . . . .

4.1.1 The CN Tower and measurement system locations. (Adapted from [48].) . 72

4.1.2 The old Rogowski coil and its location. (Adapted from [48].) . . . . . . . . 73

4.1 .3 The azimuthal magnetic field sensor. . . . . . . . . . . . . 75 
4.2.1 Digital video recording taken $2 \mathrm{~km}$ north of the $\mathrm{CN}$ Tower for the single stroke flash which occurred on August 19, 2005 at 18:37:08 (UTC). . . . .

4.2.2 Current derivative waveform for the return stroke of a single stroke flash measured on August 19, 2005 at 18:37:08 (UTC). . . . . . . . . . .

4.2.3 Current waveform for the single stroke flash which occurred on August 19,2005 at 18:37:08 (UTC). This waveform was obtained by numerically integrating the signal of Figure $4.2 .2 \ldots \ldots \ldots \ldots$

4.2.4 Azimuthal magnetic field $\left(\mathrm{H}_{\phi}\right)$ waveform observed $2 \mathrm{~km}$ north of the $\mathrm{CN}$ Tower for the return stroke of the single stroke flash measured on August 19,2005 at 18:37:08 (UTC). . . . . . . . . . . . . . . . . .

4.2.5 Vertical component of the electric field $\left(E_{z}\right)$ waveform observed $2 \mathrm{~km}$ north of the $\mathrm{CN}$ Tower for the return stroke of the single stroke flash measured on August 19,2005 at 18:37:08 (UTC). . . . . . . . . . . . . .

4.2.6 Map showing the locations of NLDN IMPACT-ESP and CLDN LPATS-IV sensors in the region around the CN Tower. The sensor location information was provided by Vaisala $[51] \ldots \ldots \ldots \ldots$

4.2.7 NALDN stroke detection efficiency plotted as a function of current wavefront maximum steepness as measured at the $\mathrm{CN}$ Tower. . . . . . . . . .

4.2.8 NALDN stroke detection efficiency plotted as a function of peak current measured at the CN Tower. . . . . . . . . . . . . . . . .

4.2.9 NALDN stroke detection efficiency plotted as a function of the peak of the azimuthal component of the magnetic field $\left(\mathrm{H}_{\phi}\right)$ measured $2 \mathrm{~km}$ north of

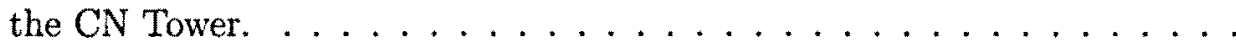

4.2.10 NALDN stroke detection efficiency plotted as a function of the peak of the vertical component of the electric field $\left(E_{z}\right)$ measured $2 \mathrm{~km}$ north of the $\mathrm{CN}$ Tower. . . . . . . . . . . . . . . . . . .

4.2.11 A distant latitude verses longitude view of the NALDN stroke location errors for the 22 detected strokes in 7 flashes. . . . . . . . . . . .

4.2.12 A close latitude verses longitude view of NALDN stroke location errors for the 22 detected strokes in 7 flashes. . . . . . . . . . . . . . .

4.2.13 North-South verses East-West distance plot of NALDN stroke location errors in kilometers. . . . . . . . . . . . . . . . . . 95

4.2.14 Histogram of the NALDN absolute location errors. . . . . . . . . . . 96

4.2.15 NALDN absolute location error plotted versus CN Tower current wavefront maximum steepness. . . . . . . . . . . . . . . . . 97

4.2.16 NALDN absolute location error plotted versus CN Tower peak current. . . 98

4.2.17 NALDN absolute location error plotted versus the peak of the azimuthal component of the magnetic field $\left(\mathrm{H}_{\phi}\right)$ measured $2 \mathrm{~km}$ north of the CN Tower. 99

4.2.18 NALDN absolute location error plotted versus the peak of the vertical component of the electric field $\left(\mathrm{E}_{z}\right)$ measured $2 \mathrm{~km}$ north of the CN Tower. . . 100

4.2.19 NALDN 50\% error ellipse semi-major axis length versus CN Tower peak current. . . . . . . . . . . . . . . . . . . 101 
4.2.20 NALDN absolute location error plotted versus NALDN $50 \%$ error ellipse semi-major axis length. . . . . . . . . . . . . . . . . . 102

4.2.21 NALDN 90\% error ellipse semi-major axis length versus CN Tower peak current. . . . . . . . . . . . . . . . . . 103

4.2.22 NALDN absolute location error plotted versus NALDN $90 \%$ error ellipse semi-major axis length. . . . . . . . . . . . . . . . . . . 104

4.2.23 NALDN 99\% error ellipse semi-major axis length versus CN Tower peak

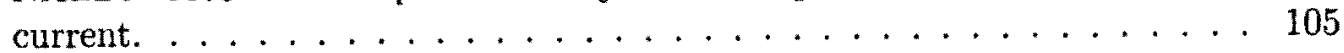

4.2.24 NALDN absolute location error plotted versus NALDN 99\% error ellipse semi-major axis length. . . . . . . . . . . . . . . 106

4.2.25 NALDN estimated peak current versus CN Tower peak current. . . . . . . 107

4.2.26 NALDN peak current estimation error $\left(\triangle \mathrm{I}=\mathrm{I}_{N A L D N}-\mathrm{I}_{C N T}\right)$ plotted versus CN Tower peak current. . . . . . . . . . . . . . . . 108 


\section{Chapter 1}

\section{Introduction}

Lightning is a common and extremely dangerous atmospheric phenomenon which is often the cause of great damage. This phenomenon has been closely studied by scientists since Benjamin Franklin first presented direct proof that thunderclouds contain electricity. The energy generated from each cloud-to-ground flash is approximately $10^{9}$ to $10^{10} \mathrm{~J}$, the approximate amount of energy it would take to operate five $100 \mathrm{~W}$ light bulbs continuously for one month [1]. Therefore, it is rather impractical to use lightning as a source of energy, particularly since most of this energy is lost as thunder, light, radio waves and heat. The main purpose for researching and studying lightning is for protection. In Canada, lightning kills an average of seven people and seriously injures 60 to 70 people per year [2]. Lightning is also responsible for approximately $42 \%$ of all forest fires, having caused loses estimated at 14 billion dollars annually between 1979 and 1993 [3]. Furthermore, lightning is the largest cause of outages on power distribution and transmission systems in lightning prone areas.

The North American Lightning Detection Network (NALDN) was developed to provide lightning protection by accurately locating lightning flashes. The NALDN allows lightning activity to be monitored in real-time for the protection of North American forests, electric power lines, and for public safety. The main applications and uses of NALDN data are [4]: 
- Weather forecasting: Help predict severe weather for public warning

- Electric power utilities: Pre-position field crews for approaching storm threats and to improve engineering and design with lightning analysis

- Air traffic control: Re-route aircraft around hazardous thunderstorms

- Airports: Suspend high-risk activities like fueling during lightning threats

- Insurance and arson: Investigate lightning as the cause of property damage or fire

- Power-sensitive manufacturing and processing operations: Prepare for storm-caused power outages by switching to back-up power early

- Hazardous materials handling: Warn personnel working near explosives and flammable materials to evacuate

- Forestry: Dispatch crews to suspected fire starts for more successful initial attack

- Golf and outdoor recreation: Warn players to seek safety from storms

- Launch facilities: Monitor for safest weather conditions for shuttle and satellite launches

Because of this vast demand for lightning protection, lightning detection has rapidly progressed from a strictly scientific and educational pursuit to a very profitable commercial venture. Clearly the NALDN is extremely important for human safety, protection of property and legal issues such as insurance claims.

In order to provide suitable protection, the NALDN estimates the following lightning information: time of stroke, location of stroke, polarity of stroke, peak current of stroke and the confidence ellipse (a percentage of certainty that a lightning event occurred within a certain distance inside the ellipse). The NALDN is composed of the U.S. National Lightning Detection Network (NLDN) and the Canadian Lightning Detection Network (CLDN). Recently the NLDN underwent an upgrade, creating a great need for the verification of its performance. Because the CN Tower is so close to the U.S. border, two out of the three 
closest lightning detecting sensors to the CN Tower are actually upgraded NLDN sensors. According to [5], measurement and validation of NALDN performance is complicated by the difficulty in obtaining definitive ground truth data. Before and after the recent NLDN upgrade, evaluations using video cameras, electric field recordings, and optical recordings were performed by the University of Arizona in [6] and [7]. The University of Florida's International Center for Lightning Research and Testing (ICLRT) at Camp Blanding used rocket-triggered lightning to provide ground truth data in [8]. According to [5], media and eyewitness reports of lightning strikes have also been used to verify network performance.

The CN Tower provides an excellent opportunity to evaluate the performance characteristics of the NALDN in the Toronto area. The CN Tower is one of the best sites in the world to observe the lightning phenomenon at tall structures and to obtain definitive lightning data such as currents, magnetic and electric fields, and visual parameters. Lightning strikes to the CN Tower have been observed since 1978, and although the lightning flash density is less than 2 flashes per square kilometer in Toronto, the CN Tower receives several tens of strikes each year, providing a good opportunity to evaluate NALDN performance [9]. In fact, the CN Tower allows for an evaluation of every measurement provided by the NALDN, including the peak current of each stroke. The only other evaluation which was capable of doing this was the rocket-triggered evaluation performed in Florida at Camp Blanding in [8].

This thesis presents a comprehensive evaluation of the NALDN performance characteristics in the Toronto area. The data from CN Tower lightning strikes from the lightning season in 2005 are used to evaluate NALDN performance characteristics such as: flash detection efficiency, stroke detection efficiency, absolute location error, peak current estimation and location accuracy model $(50 \%, 90 \%$ and $99 \%$ error ellipse) error.

In Chapter 2, the basic theory behind lightning locating and detecting is introduced. The varying lightning emissions that occur during the different physical processes in a lightning 
flash are described initially, along with the influences which affect electromagnetic wave propagation through the atmosphere. Based on these emissions, various lightning locating techniques have been invented, but this chapter focuses solely on the techniques utilized by the NALDN, providing an introduction to the lightning locating techniques used in the network.

The NALDN is closely studied in Chapter 3. Its history, technology, current operational status, effectiveness at lightning detection, and its applications are examined. Most importantly, the results from other recent performance evaluations of the NALDN are presented and analyzed in this chapter.

In Chapter 4, the results of the NALDN evaluation in the Toronto area are presented. Initially the CN Tower lightning project is discussed and a detailed description is given of the equipment used to acquire lightning data. Afterwards, the performance characteristics of the NALDN are evaluated using the CN Tower lightning data acquired over the Toronto lightning season in 2005. A detailed analysis of the NALDN flash detection effciency, stroke detection efficiency, absolute location error, peak current estimation and location accuracy model (50\%, 90\% and 99\% error ellipses) is given, followed by an in-depth discussion of the results.

Lastly, conclusions and recommendations are given in Chapter 5. 


\section{Chapter 2}

\section{Lightning Location Basics}

This chapter provides an introduction to the science of lightning location. Initially, the different lightning emissions which occur from the various physical processes involved in lightning flashes are examined. Based on the emissions that occur from lightning flashes, certain techniques may be employed in order to detect and locate a lightning flash. But before discussing these techniques, it is necessary to also examine how electromagnetic wave propagation is influenced by the atmosphere and surrounding areas, in order to better understand how lightning emissions may be affected in different environments.

Subsequently, the basics behind the lightning detection methods used in the North American Lightning Detection Network (NALDN) are described. In the NALDN, lightning locating and detecting methods are based magnetic field direction finding and time-of-arrival techniques. It should be noted that this chapter only introduces the basic principles behind the two important lightning locating techniques, in order to familiarize the reader with how they function. The complex methods by which these technologies actually operate in the NALDN, along with the algorithm currently used to identify the location of a stroke, is later described in Section 3.4.1. 


\subsection{Lightning Emissions}

Many physical processes occur in cloud-to-cloud and cloud-to-ground lightning flashes, and each process produces a characteristic electric and magnetic field. As shown in Figure 2.1.1, lightning emits significant electromagnetic energy in the frequency range from 1 to 300 $\mathrm{MHz}$, with a spectral peak at around 5 to $10 \mathrm{kHz}$ for lightning at distances over $50 \mathrm{~km}$. [1].

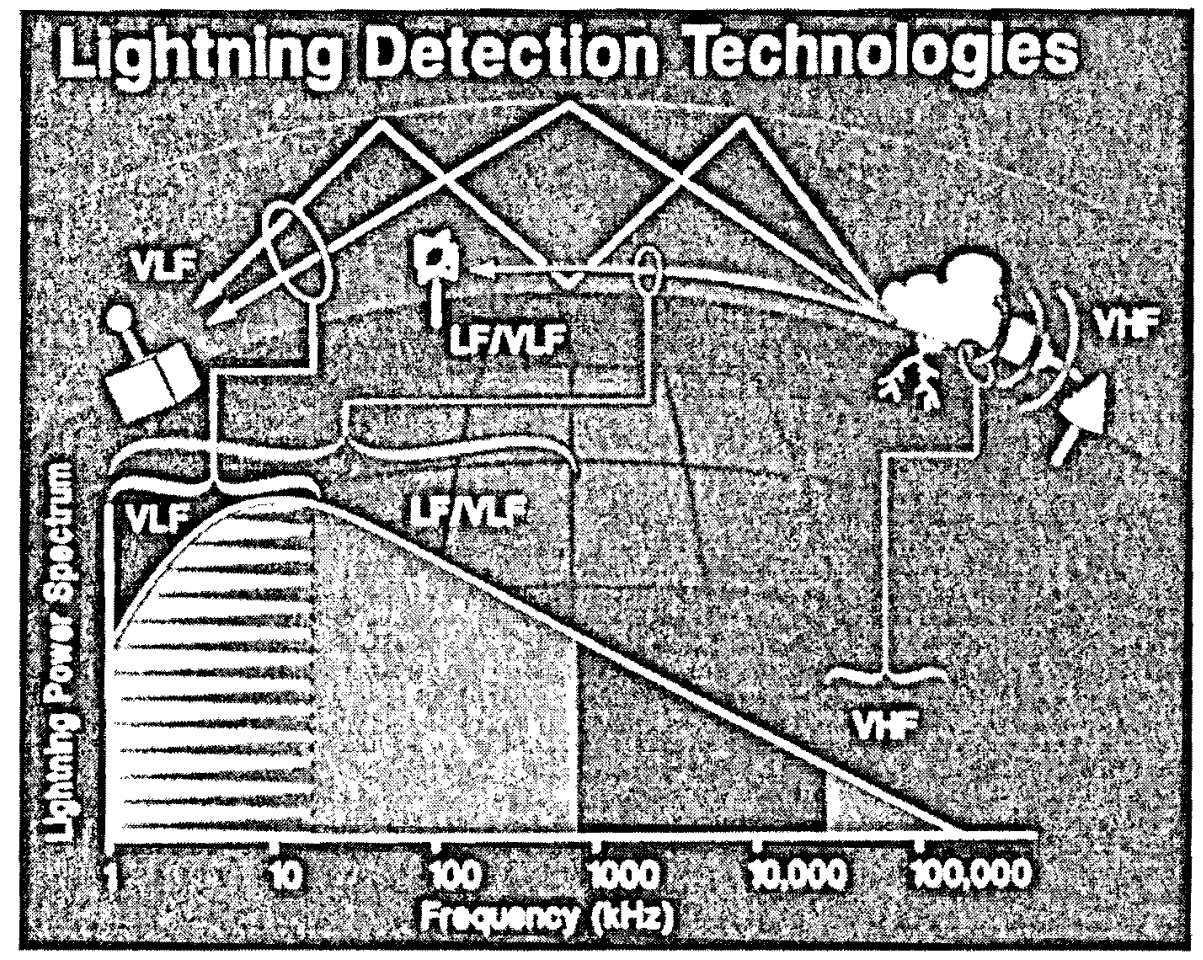

Figure 2.1.1: Relationship between frequency and lightning, along with the various VLF; LF, and VIIF lightning detection technologies. (Adapted from [10].)

It should be noted that electromagnetic radiation from lighting is detectable at even higher frequencies from $300 \mathrm{MHz}$ to $300 \mathrm{GHz}$, and in the visible light frequencies from $10^{14}$ to $10^{15} \mathrm{~Hz}[1]$. In addition to electromagnetic radiation, lightning also produces acoustic radiation.

During the lightning process, significant electromagnetic and acoustic radiation is gener- 


\subsection{Lightning Emissions}

Many physical processes occur in cloud-to-cloud and cloud-to-ground lightning flashes, and each process produces a characteristic electric and magnetic field. As shown in Figure 2.1.1, lightning emits significant clcctromagnetic energy in the frequency range from 1 to 300 $\mathrm{MHz}$, with a spectral peak at around $\lrcorner$ to $10 \mathrm{kHz}$ for lightning at distances over $50 \mathrm{~km}$. [1].

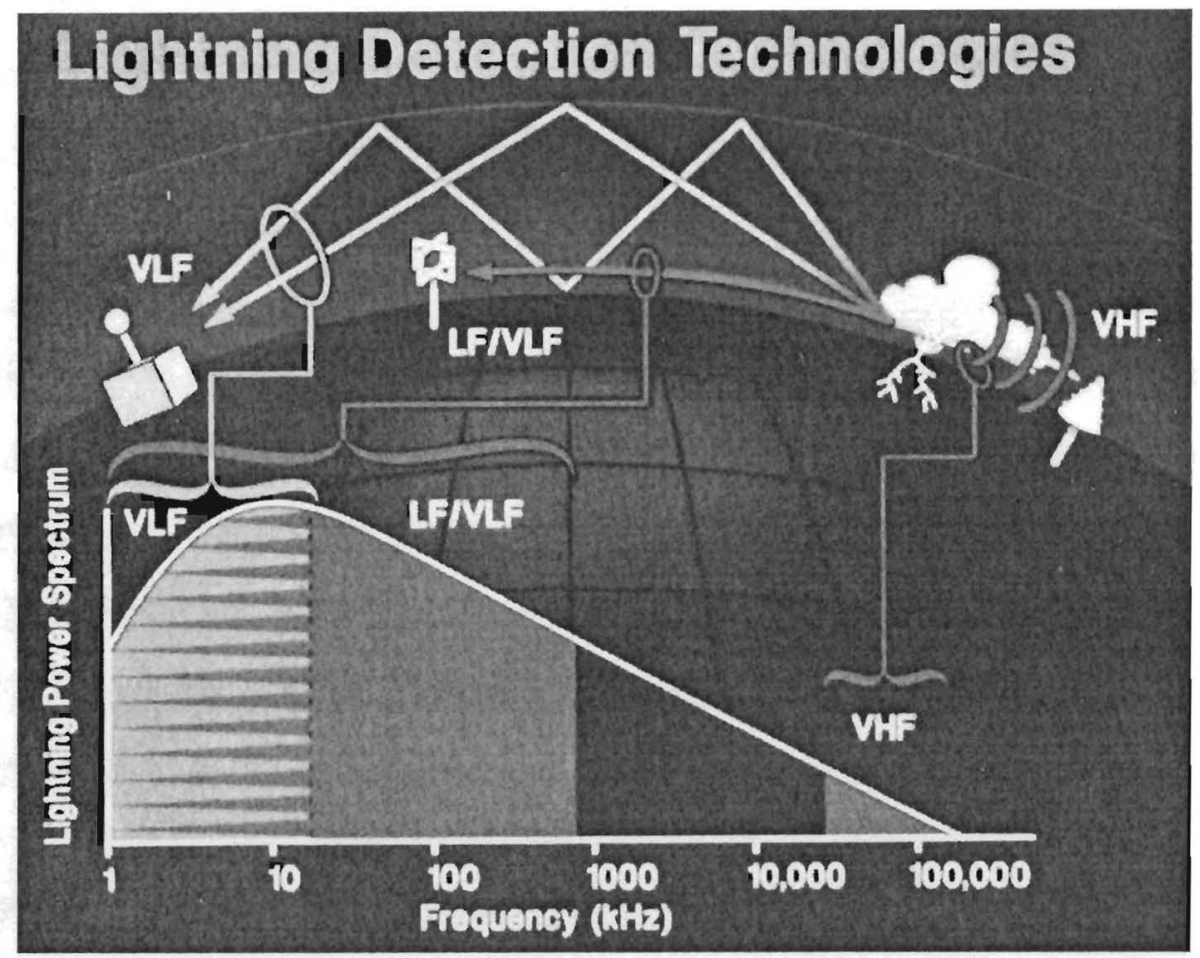

Figure 2.1.1: Relationship between frequency and lightning, along with the various VLF, $\mathrm{LF}$, and VHF lightning detection technologies. (Adapted from [10].)

It should be noted that electromagnetic radiation from lighting is detectable at even higher frequencies from $300 \mathrm{MHz}$ to $300 \mathrm{GHz}$, and in the visible light frequencies from $10^{14}$ to $10^{15} \mathrm{~Hz}$ [1]. In addition to electromagnetic radiation, lightning also produces acoustic radiation.

During the lightning process, significant electromagnetic and acoustic radiation is gener- 
ated as follows:

- Radio Frequency (RF)

- During breakdown and ionization processes (mostly from leaders and streamers), there are strong emissions in the VHF (30 to $300 \mathrm{MHz}$ ) range [10]. When high currents occur in previously ionized channels (mostly from return strokes and the active stage of cloud flashes), the most powerful emissions occur in the LF (30 to $300 \mathrm{kHz}$ ) and VLF ( 3 to $30 \mathrm{kHz}$ ) ranges [10]. The electromagnetic radiation generated by cloud-to-cloud and cloud-to-ground lightning is schematically depicted in Figure 2.1.2.

- Optical

- Visible light $\left(10^{14}\right.$ to $\left.10^{15} \mathrm{~Hz}\right)$ is emitted for a few milliseconds because of the thermal radiation of the hot (up to $30000 \mathrm{~K}$ ) lightning channel [11].

- Acoustical

- Thunder is produced by the rapidly expanding hot air along the lightning path.

Because of their channel length and large currents, cloud-to-ground return strokes completely dominate the VLF and LF radiation fields produced by lightning, consequently creating only a few large pulses per flash [10]. Conversely, cloud-to-cloud flashes create tens to hundreds of small pulses in the LF range, but the amplitude of these pulses is only about $5 \%$ of the median amplitude of the return stroke pulses, with pulses of comparable magnitude occurring very rarely [10].

There are approximately a hundred times as many pulses in the VHF range as in the LF and VLF ranges, with the amplitudes of the pulses produced by cloud-to-cloud flashes being comparable to those of cloud-to-ground flashes. [10]. The VHF radiation is produced by breakdown processes with small currents and dimensions of tens to hundreds of meters [12]. High current lightning processes such as return strokes emit very little VHF radiation. Since the various physical processes in cloud-to-cloud and cloud-to-ground lightning produce 


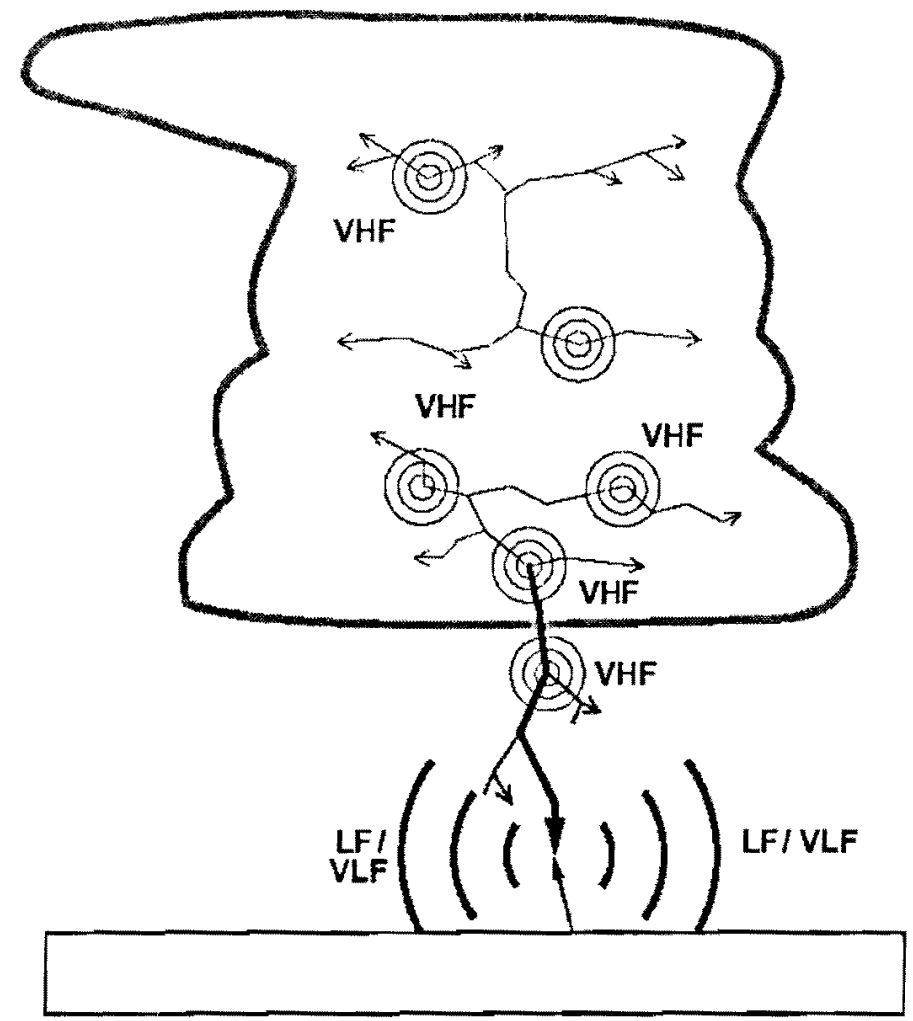

Figure 2.1.2: Electromagnetic radiation generated by the different physical processes in lightning. (Adapted from [11].)

electromagnetic radiation with different frequencies and characteristics, numerous lightning locating techniques have been developed.

\subsection{Influences on Electromagnetic Wave Propagation through the Atmosphere}

Before discussing lightning locating techniques, it is necessary to briefly mention the factors which influence electromagnetic wave propagation through the atmosphere, in order to better understand how the pulses produced by lightning can be affected. The propagation of electromagnetic waves in the atmosphere is affected by the following factors [11]: 
- Attenuation

- Assuming no obstructions, an electromagnetic wave loses power in proportion to the distance that it travels through the atmosphere.

- Ground reflections

- These reflections depend on the properties of the soil. Propagation is also affected by large mountain ridges which delay the radiated signal. Obstacles which obscure a part of they sky will also limit the ability to detect a signal. Additionally, any metallic objects close to a sensor can distort the magnetic field.

- Ionospheric reflections

- Electromagnetic waves will interact with the highly conductive ionosphere. Electromagnetic waves with frequencies smaller than the ionospheric cutoff frequency (which ranges from 4 to $9 \mathrm{MHz}$ ) will reflect back towards the ground [13]. Therefore, long waves can propagate in the waveguide formed by the ground and ionosphere, travelling long distances with low energy loss. Waves with frequencies above the ionospheric cutoff frequency and up to $30 \mathrm{MHz}$ are generally refracted and returned to the earth's surface [13]. Signals above $30 \mathrm{MHz}$ usually penetrate the ionosphere [13].

- Dispersion, scattering, refraction, absorption

- These factors distort the wave front and change the frequency spectrum of the electromagnetic wave.

Electromagnetic waves with frequencies higher than $80 \mathrm{MHz}$ propagate approximately within the line-of-sight, whereas electromagnetic waves with frequencies lower than the ionospheric cutoff can propagate for long distances as their energy is trapped in the atmospheric waveguide [11]. 


\subsection{Lightning Detection Methods used in the North American Lightning Detection Network}

The main purpose of the NALDN is to accurately locate the ground strike point of cloudto-ground lightning. As later described in Section 3.7, this information is very important for insurance and liability claims, and especially for the protection of life and property. Cloudto-ground discharges are typically detected using the strong VLF and LF signals generated by return strokes. The locating systems based on VHF signals have a limited detection range because of the line-of-sight propagation of the signals, as mentioned in Section 2.2. Therefore, it is obvious that VLF/LF systems are better suited for the detection of cloud-to-ground lightning strikes, since the VLF/LF components of a lightning discharge will propagate for long distances along the earth's surface or in the earth-ionosphere waveguide as shown in Figure 2.1.1. This allows detection sensors to be placed further apart from each other, at reasonable distances.

The NALDN network currently uses a combination of magnetic direction finders (DFs) and time-of-arrival (TOA) sensors to locate lightning. Both of these sensors operate in the VLF and LF range. The DF and TOA technology used in the NALDN is closely examined in the following sections. Because the technologies were developed separately and can function independently of each other, each technology is described separately at first and the hybrid system used in the NALDN is discussed later on in Section 3.4.1. Although the algorithms and locating methods discussed for each independent technology are now outdated because of the hybrid system used in the NALDN, the basic ideas behind the technologies remain the same. Therefore, it is necessary to review the development of the magnetic DF and TOA technology independently in order to have a comprehensive understanding of how the techniques function together in the hybrid system.

A description of the other lightning locating techniques such as: charge center analysis, 
lightning location with radar, acoustic mapping techniques, VHF radio mapping techniques and satellite mapping techniques can be found in [1], [10], [11], and [14].

\subsubsection{Magnetic Field Direction Finding}

The basic idea behind magnetic field direction finding is to measure the magnetic field of a lightning strike using a crossed-loop antenna with two vertical loops mounted perpendicular to each other; one loop is oriented north-south while the other loop is oriented east-west [14]. An assumption is made that the lightning strike being located is completely vertical, producing a magnetic field which only has an azimuthal component. The output voltage induced in each vertical loop, by Faraday's law, is proportional to the derivative of the magnetic field and to the cosine of the angle between the magnetic field vector and the normal vector to the plane of the loop [1].

If a vertical lightning strike occurs directly north or south of the sensor, clearly the northsouth loop will receive a maximum signal while the east-west loop of the sensor will receive no signal. The signal in the north-south loop varies as the cosine of the angle between north and the lightning source as viewed from the antenna, while the signal in the east-west loop varies as the sine of the same angle [1]. The ratio of signals is proportional to the tangent of the angle between north and the lightning source as viewed from the antenna (the azimuth angle to the source) [1]. Therefore, the signal induced in the loops is dependent on the current in lightning strike, the distance from the strike to the sensor, and the azimuth of the strike with respect to the sensor. The ratio of the signals induced in the loops provides the azimuth angle, which points towards the lightning source. It should be noted that positive strikes and negative strikes will induce the same signal polarity, making them indistinguishable.

There are two types of magnetic DFs: narrowband DFs and gated wideband DFs. Narrowband DFs have been used for lightning detection since the 1920 s and are described in [1]. 
As described in [1], the major disadvantages of narrowband DFs are the inherent polarization errors (angle errors) which occur for close range lightning (lightning less than $200 \mathrm{~km}$ from the sensor). The inherent and uncorrectable errors are mainly caused by non-vertical lightning channels and skywaves which are produced by reflections from the ionosphere, as mentioned in Section 2.2. Both of these create non-vertically oriented magnetic fields.

Gated wideband DFs were developed in the early 1970s to overcome the problem of large polarization errors at short ranges. The company Lightning Location and Protection, Inc. (LLP) was formed by E.P. Krider, M.A. Uman and A.E. Pifer in the mid-1970s to develop commercial gated wideband magnetic DFs [1]. The history of the NALDN, including the development and use of magnetic DFs in the network is discussed in Section 3.1.

The gated wideband DF described in [15], was designed so that only part of the lightning signal generated by a cloud-to-ground strike is detected. This system operated in the time domain covering the LF and VLF ranges from 1 to $500 \mathrm{KHz}$, and was designed to respond to field waveforms that were characteristic of the return strokes in cloud-to-ground flashes [10]. The magnetic field was sampled in the north-south and east-west loops at the initial peak of the return stroke magnetic field [10]. This peak is radiated from the bottom hundred meters of the lightning channel, during the first microseconds of the return stroke [1]. Because the bottom of a lightning channel tends to vertical, the magnetic field will be horizontal. Furthermore, a gated wideband DF doesn't record ionospheric reflections, since those reflections arrive long after the initial peak magnetic field is sampled [1]. Therefore, the errors inherent in the narrowband DFs were no longer a problem for the gated wideband DFs.

As previously mentioned, a DF system will not distinguish between positive and negative strokes. Consequently, the gated wideband DF only detected negative ground flashes until the late 1980s [1]. Positive and negative strokes induce the same signal polarity, causing a $180^{\circ}$ ambiguity in the location of the stroke [13]. To account for this, in the 1980 s the electric field began to also be sampled in order to determine the stroke polarity and remove 
this ambiguity. Figure 2.3.1 shows the components of an LLP magnetic DF station from the Ontario provincial lightning location system (LLS) in 1989.

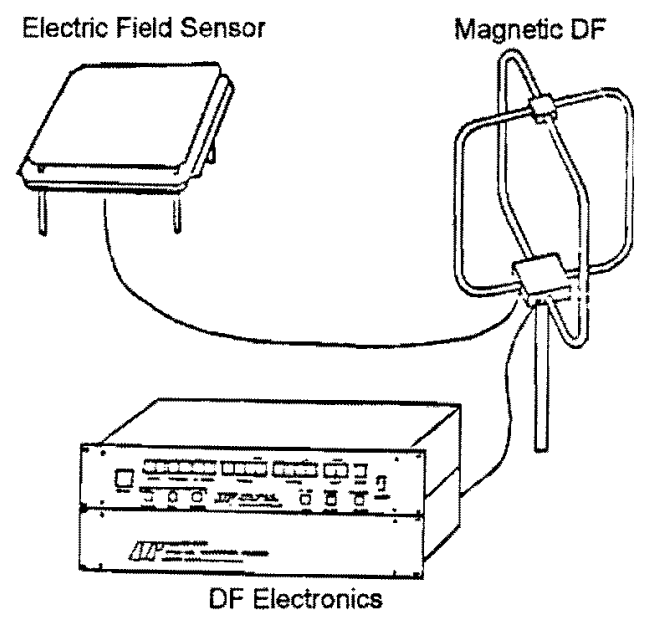

Figure 2.3.1: Components of a LLP magnetic DF station used in the Ontario provincial LLS in 1989. (Adapted from [13].)

Site errors are the largest sources of error in the magnetic DF system. Site errors are caused by the presence of unwanted magnetic fields due to non-flat terrain and nearby conducting objects such as underground and overhead power lines which are excited to radiate by the incoming lighting fields [1]. Site errors are a systematic function of direction but are generally time invariant [1]. Usually the DF site errors are determined and then the angle corrections can be made in real-time [14]. In order to correct site errors, the data from a network of at least three DFs is recorded and analyzed for one to three months to determine a site correction curve as a function of angle for each DF [1]. In the recent 2003 upgrade of the U.S. National Lightning Detection Network (NLDN), site errors can be analyzed and corrected must faster, as described later on in Section 3.3.

Magnetic DF systems in LLP networks typically used two or three sensors to determine the location of a lightning strike. As previously mentioned, each sensor produces a direction vector which points towards the lightning source. As shown in Figure 2.3.2, when two sensors 
are used the location of the lightning stroke is found by simple triangulation. The location contains some error because each direction vector may have some random angular error and perhaps some site error. In Figure 2.3.2, each sensor has a $\pm 1^{\circ}$ azimuth error.

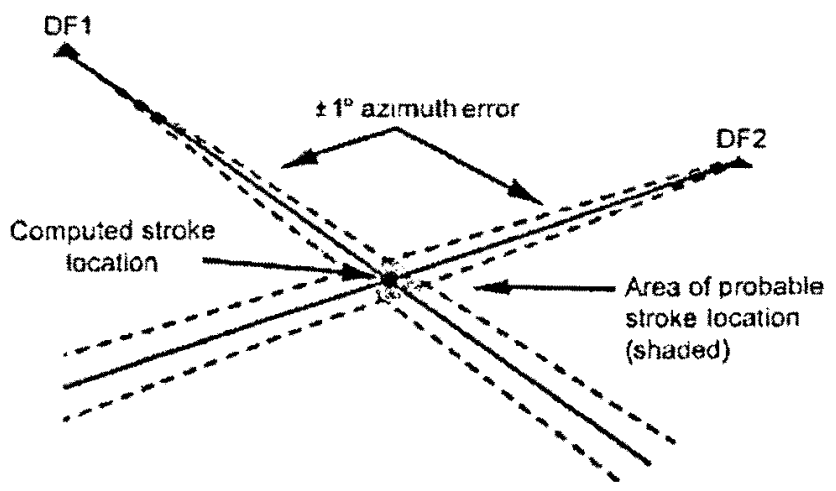

Figure 2.3.2: Determination of a lighting stroke location using only two DFs. (Adapted from [1].)

As shown in Figure 2.3.3, if a strike occurs along a line between only two sensors, large azimuth errors can result in significant location error, perhaps resulting in no intersection of the direction vectors at all [10]. In Figure 2.3.3, the stroke is assumed to be on the baseline and the ratio of the signal strengths is used to determine its position [1]. Because of this baseline problem, practical networks use at least three sensors.

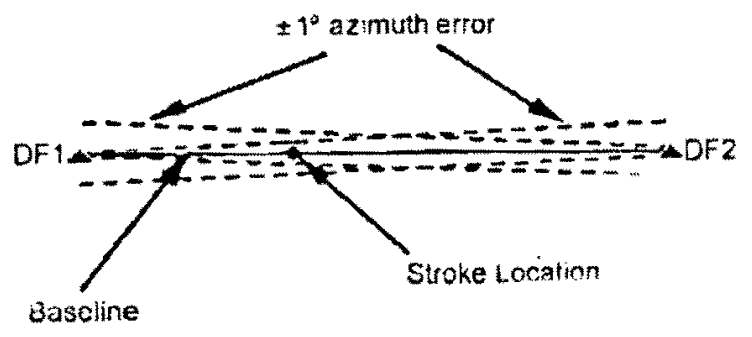

Figure 2.3.3: Baseline problem that may occur when using only two DFs to detect a stroke. (Adapted from [1].)

In Figure 2.3.4, three magnetic DFs are used to locate a stroke. Each pair of DFs provides a location resulting in three possible stroke locations. The distance between the locations 
provides some measure of the system error.

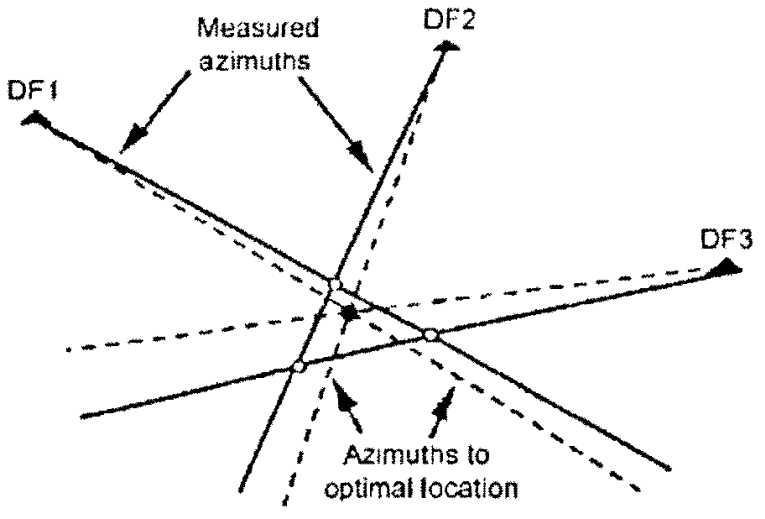

Figure 2.3.4: Determination of a lighting stroke location using three DFs. (Adapted from [1].)

When three or more DF sensors are used to locate a stroke, the optimal estimate of the stroke location is found by a nonlinear least-squares technique, which involves minimizing the following $\chi^{2}$ function [1]:

$$
\chi^{2}=\sum_{i=1}^{N}\left(\frac{\theta_{m i}-\theta_{i}}{\sigma_{\theta_{i}}}\right)^{2}+\sum_{i=1}^{N}\left(\frac{E_{m i}-E_{i}}{\sigma_{E_{i}}}\right)^{2}
$$

where $\theta_{i}$ and $E_{i}$ are the unknown azimuth and electric field peak values, $\theta_{m i}$ and $E_{m i}$ are the azimuth and electric field peak measured at the $i$ th station, and the $\sigma$ 's are the measurement error estimates.

The values of the unknowns $\theta_{i}$ and $E_{i}$, found by the minimization of $\chi^{2}$ in Equation 2.3.1, provide the most probable location of the stroke and also allow for the estimation of the errors in this most probable location [1]. The error estimates correspond to certain confidence ellipses, where within the ellipse there is a probability that the stroke was located. The determination of confidence ellipses by assuming the errors in the measured parameters are Gaussian is discussed in Section 3.5.1. This approach implies that the major errors (such as site errors) are eliminated, leaving the random errors, number of DFs used, and network geometry to determine the size of the confidence ellipses [1]. 
When the U.S. NLDN was formed in 1989, its sensors consisted of only LLP magnetic DFs [1]. Currently the NALDN also uses time-of-arrival (TOA) technology (which is described below in Section 2.3.2) along with the magnetic DF technology. The hybrid lightning locating technique used in the NALDN is described later on in Section 3.4.1.

\subsubsection{Time-of-arrival technique}

A time-of-arrival (TOA) sensor provides the time at which some portion of the lightning electromagnetic field arrives at the sensing antenna [1]. There are three types of TOA systems for locating lightning [1]:

- very-short baseline (tens to hundreds of meters)

- short-baseline (tens of kilometers)

- long-baseline (hundreds to thousands of kilometers)

The very-short and short-baseline systems operate at VHF, while the long-baseline systems operate at VLF and LF. As mentioned in Section 2.1, VHF radiation is associated with breakdown processes, while VLF and LF signals are associated with the high currents from lightning return strokes. According to [1], short-baseline systems are used to provide images of lightning channels and to study the spacial and temporal development of discharges. The long-baseline system is used to identify the strike point of cloud-to-ground lightning and is the main focus of this section.

The first long-baseline TOA system which operated at VLF and LF was described in [16]. The lightning positioning and tracking system (LPATS) is a commercial long-baseline TOA system developed in the 1980s by the Atlantic Scientific Corporation, which later became Atmospheric Research Systems, Inc. (ARSI) [1]. The LPATS operates at LF and VLF, using electric field whip antennas at four or more stations separated by 200 to $400 \mathrm{~km}$ [1]. The system uses a TOA technique where each station identifies the TOA of an electric field 
signal from lightning, and then a central processor determines the differences in the times that the lightning signal arrived at the stations [14].

The difference in the TOA for a pair of stations defines a locus of constant time difference that passes through the lightning stroke location [14]. For stations on a flat plane the locus is a hyperbola, but for ranges at which the curvature of the Earth must be taken into account the locus becomes distorted from it's hyperbolic shape [14]. A third station provides a second independent time difference, and the locus of this second time difference intersects the first locus at the point at which the lightning stroke occurred [14]. However, the hyperbolae on the earth's surface from only two time differences may intersect at two different points under some geometrical conditions as shown in Figure 2.3.5, creating ambiguity regarding the location of the lightning stroke. Only one point in Figure 2.3.5 corresponds to the true stroke location, while the other point is insignificant. In this case, a fourth TOA sensor is needed to provide a third time difference and a third hyperbola to detect the true stroke location.

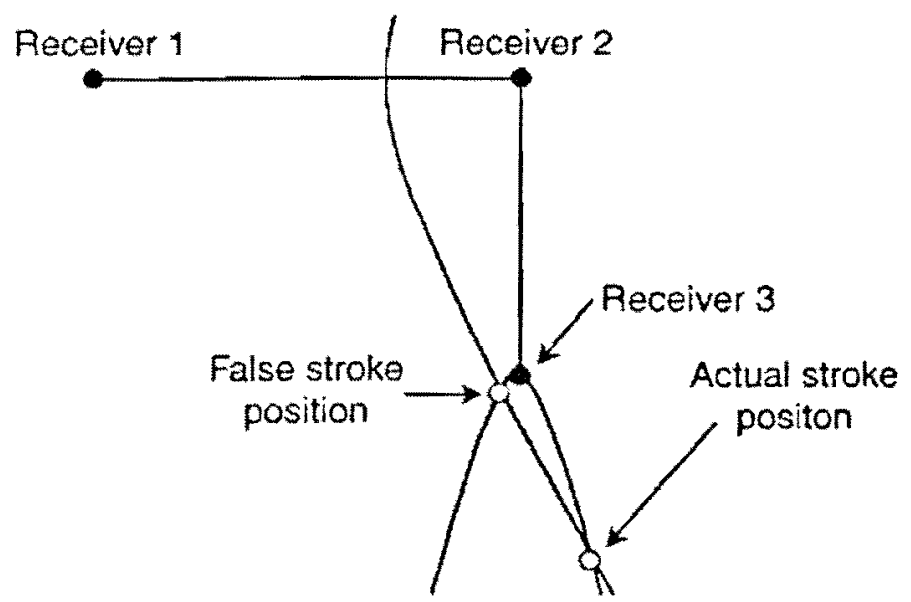

Figure 2.3.5: Determination of a lighting stroke location using three TOA sensors when the solution is not unique. (Adapted from [1].)

For cloud-to-ground lightning near or within a network, there is usually only one solution 
as shown in Figure 2.3.6; therefore, LPATS determines the location of the stroke by only solving for the intersection of the two hyperbolas found from the three sensors [1]. If possible, this location is verified by redundant data.

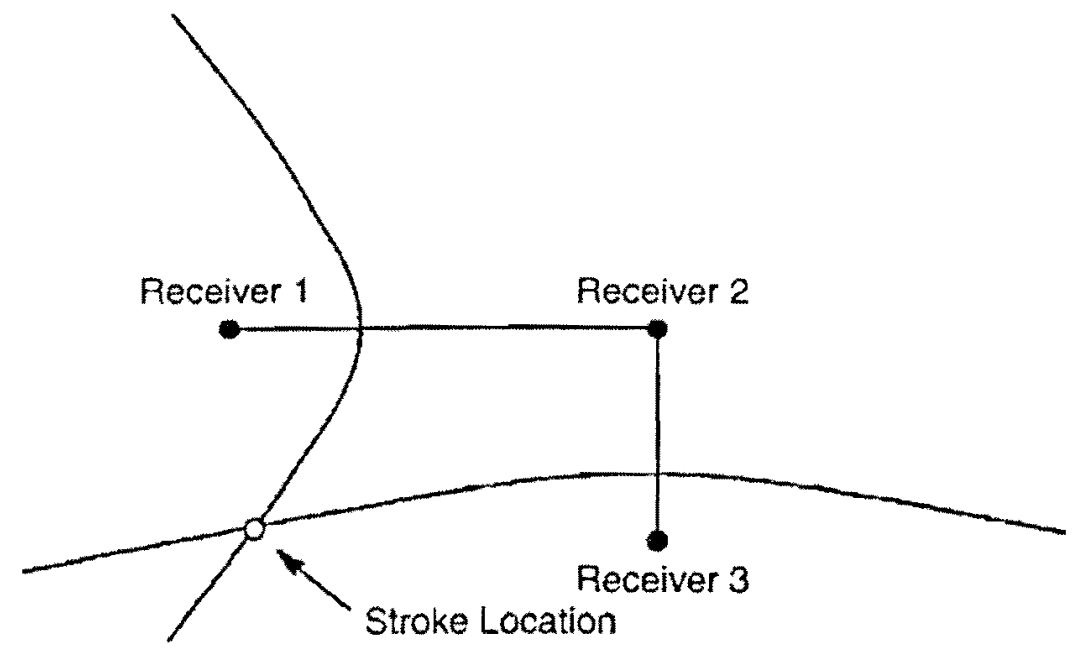

Figure 2.3.6: Determination of a lighting stroke location using three TOA sensors when the solution is unique. (Adapted from [1].)

Each LPATS station consists of a time signal receiver, a time signal generator, a lightning stroke detector, and a vertical antenna to receive reference timing signals and sense electric field changes from lightning. [14]. The essential task for TOA systems is to accurately measure the arrival time of the same part of a lightning signal at all the sensors that are used to detect the stroke. When the sensors are over $200 \mathrm{~km}$ apart, the time between stations should be synchronized within less than $10^{-6} \mathrm{~s}$ in order to achieve good performance [14]. Originally the LORAN-C or other Earth based standard timing signals were used at each of the individual sensors.

Locating errors in LPATS may be caused by anything that changes the arrival time of an ideal signal such as: signal distortion in propagation (which causes the wrong part of a waveform to be identified at a sensor), path elongation due to mountains, and inadequate time synchronization between stations [1]. Most of the problems in the early version of 
LPATS were corrected with the adoption of GPS timing. The most recent version of LPATS can include extensive waveform storage capabilities, which can be used to implement criterions for identifying flash types [14]. Currently LPATS processes the signal to identify the beginning of the pulse for ground flashes, which corresponds to the return stroke [14].

As previously mentioned, the NALDN uses TOA technology in conjunction with magnetic DF technology for lightning location. The method used in the NALDN does not involve finding the TOA hyperbolae as mentioned above. This section was provided as a historical reference to understand how the TOA technology functioned independently to locate strokes in the ARSI network. In the NALDN, the TOA is simply used to create a range circle around a sensor, which depicts the possible radial range at which a lightning stroke has occurred [10]. The absolute arrival time of the lightning discharge electric field is measured with a GPS timing system at each sensor, providing an estimate of the location and time of the lightning discharge. Therefore, each sensor provides information which establishes the radial range of the discharge from the sensor. The estimated range of the lightning event is based on the difference between the estimated time of the lightning discharge and the measured time of its arrival at the sensor site. The magnetic DF still provides azimuth information, as mentioned in the previous section. This new method, including the algorithm behind it, is discussed later on in Section 3.4.1.

\subsection{Summary}

This chapter provided an introduction to the fundamentals of lightning location. Lightning emits significant electromagnetic energy in the frequency range from 1 to $300 \mathrm{MHz}$, with a spectral peak at around 5 to $10 \mathrm{kHz}$ for lightning at distances over $50 \mathrm{~km}$. In fact, during the lightning process electromagnetic radiation is generated in the RF and optical spectrum range, and acoustic radiation is also generated. Because of their channel length 
and large currents, cloud-to-ground return strokes completely dominate the VLF and LF radiation fields produced by lightning, consequently creating only a few large pulses per flash. There are approximately a hundred times as many pulses in the VHF range as in the LF and VLF ranges, with the amplitudes of the pulses produced by cloud-to-cloud flashes being comparable to those of cloud-to-ground flashes. The VHF radiation is produced by breakdown processes with small currents and dimensions of tens to hundreds of meters.

The propagation of electromagnetic waves in the atmosphere is affected by attenuation, ground reflections, ionospheric reflections, dispersion, scattering, refraction and absorption. Based on the emissions that occur from lightning flashes, certain techniques may be employed in order to detect and locate a lightning flash. The NALDN uses magnetic field DF and TOA techniques to detect and locate lightning. In the magnetic DF technique, the magnetic field of a return stroke is measured with two crossed loop antennas for the determination of a direction vector which points towards the stroke. Historically, the TOA sensors worked by measuring the TOA difference of an electric field signal from lightning between different sensors, producing a hyperbolic curve. In the NALDN, the absolute arrival time now provides the range information. This chapter only introduces the basic principles behind the two important lightning locating techniques, the complex methods by which these technologies actually operate in the NALDN, along with the algorithm currently used to identify the location of a stroke, is later described in Section 3.4.1. 


\section{Chapter 3}

\section{The North American Lightning}

\section{Detection Network}

In this chapter, a comprehensive overview of the North American Lightning Detection Network (NALDN) is provided. Initially a historical overview of the network is given, detailing how and why such a large lightning detection network came into existence so rapidly. Afterwards the development of the network and its technology is discussed, depicting how the network evolved from the United States to include Canada as well, and discussing the significant sensor upgrades along the way. Naturally the operations and communications of the NALDN is discussed next, detailing the lightning measurements acquired by the network and the manner by which the data is analyzed and distributed for real-time utilization by customers. Following this, the lightning locating technology and algorithms used in the NALDN are thoroughly examined. The methods by which lightning strokes are detected, located, grouped into flashes, assigned the correct polarity and timing, and have their peak currents estimated are discussed.

In order to evaluate and predict the performance of different sensor configurations in the NALDN, location accuracy and detection efficiency models were developed. These models 
are also discussed and comprehensively analyzed in this chapter. Afterwards, the past and present performance of the NALDN is analyzed, including the recent results obtained from other performance evaluations of the NALDN. Lastly, the main applications that utilize NALDN data are mentioned, to further underscore the importance and value of this network.

\subsection{History}

In the mid-1970s, E.P. Krider, M.A. Uman and A.E. Pifer were researching the use of magnetic DF technology for lightning location at the University of Arizona. Because of the numerous lightning related forest fires in Alaska, the U.S. Government's Bureau of Land Management (BLM) had asked the scientists to devise a sensor which could effectively detect and locate lightning strikes. The gated wideband magnetic DF sensor was the outcome of their research [17]. The following description of the formation of the U.S. National Lightning Detection Network (NLDN) is summarized from [1] and [18].

The company Lightning Location and Protection, Inc. (LLP) was formed by E.P. Krider, M.A. Uman and A.E. Pifer in the mid-1970s to commercially develop gated wideband magnetic DFs. The first commercial magnetic DF networks required that the DF vectors at each station be recorded and then combined to determine the lightning location. In fact, the magnetic DFs described in [19], which were first used in Alaska in 1976 for forest fire detection, required that the operators be in phone contact with each other in order to determine the lighting location in real-time.

From 1976 to 1996 , the BLM developed an LLP magnetic DF network covering 11 states in the western United States and Alaska for the purpose of forest fire detection. The LLP magnetic DF networks in the mid-1980s were typically composed of three stations commu-

nicating to a central position analyzer (a computer which processed the magnetic DF data to determine location, probable error, and various lightning stroke parameters) by dedicated 
telephone lines. Starting in 1979, the U.S. National Severe Storm Laboratory (NSSL) developed a magnetic DF network which covered Oklahoma and parts of adjacent states. From 1982 to 1986, researchers at the State University of New York at Albany (SUNYA) established the U.S. East Coast network using magnetic DFs. By 1989, lightning strikes in the contiguous United States were being detected in real-time by an integrated system composed of these three magnetic DF networks, this system was named the U.S. National Lightning Detection Network (NLDN).

The main reason for developing the NLDN began in 1983, when the electric power industry recognized the advantages of locating cloud-to-ground lightning. This led the Electric Power Research Institute (EPRI) to fund the expansion and operation of the SUNYA U.S. East Coast network. The origin of the NLDN begins in 1987, when researchers at SUNYA combined data from the BLM, NSSL and SUNYA regional networks of LLP gated wideband magnetic DFs on an experimental basis. The three separate networks were combined into the NLDN with the assistance and encouragement of the U.S. Federal Coordinator for Meteorological Services.

In 1989, around the same time as the NLDN began real-time operation, an independent commercial nationwide network of time-of-arrival (TOA) sensors was being installed. Back in 1980 , the first commercial long-baseline TOA system called the lightning positioning and tracking system (LPATS) was developed independently by the Atlantic Scientific Corporation, which later became Atmospheric Research Systems, Inc. (ARSI). By the late 1980s, a network of LPATS sensors was installed nationwide by ARSI; the ARSI network was composed of 11 regional clusters which had six TOA sensors each, with the sensors typically separated by 200 to $400 \mathrm{~km}$.

Due to the growing commercial interest in nationwide lightning data, the company GeoMet Data Services, Inc. (GDS) was formed in 1991 by LLP and the ERPI to commercially distribute lightning data from the NLDN of magnetic DFs. According to [17], the 
Sankosha Corporation of Japan purchased and reorganized LLP, GDS and ARSI to form Global Atmospherics, Inc. (GAI) in 1995. Global Atmospherics was based in the U.S. in Tucson, Arizona. Previously in 1992, LLP had developed the Improved accuracy from combined technology (IMPACT) method, which utilizes both magnetic DF and TOA data to locate strikes. This method can employ information from TOA sensors, magnetic DF sensors, and IMPACT sensors (which combine TOA and DF technology all in one sensor). In the 1995 upgrade to the NLDN, the two major lightning locating technologies were combined by GAI to provide an increased detection efficiency (DE) and almost an order of magnitude improvement in locating accuracy.

Before February 1998, lightning occurrence information in Canada was available only from manned surface stations and a few provincial networks with limited coverage [20]. As shown in [13], Ontario had a provincial network consisting of 16 LLP magnetic DF sensors in 1989. In order to enhance the predictive capability within the Severe Weather Program, the Meteorological Service of Canada (MSC) contracted GAI in 1997 to implement the Canadian Lightning Detection Network (CLDN) [21]. Global Atmospherics supplied and installed the sensors for the CLDN, and began to operate the network for Environment Canada in 1998 [3]. Therefore, the North American Lightning Detection Network (NALDN) was created by combining the U.S. National Lightning Detection Network (NLDN) with the new Canadian Lightning Detection Network (CLDN) in 1998.

In March 2002, the Vaisala Group from Finland acquired GAI from the Sankosha Corporation [22]. It should be noted that although the CLDN is owned by Environment Canada it is operated by Vaisala and it is the only national lightning network in Canada [23]. The XALDN detects the electromagnetic signal from a lightning strike, and each sensor transmits the lightning information via satellite to the Network Control Center (NCC) in Tucson, Arizona, where the information is processed [23]. The U.S. NLDN recently underwent another network upgrade in 2003. This upgrade, as well as the development of the whole NALDN is 
discussed in the next section.

\subsection{Development and Sensor Locations}

As previously mentioned, the U.S. NLDN has provided lightning detection covering the continental United States since 1989 using gated wideband magnetic DF sensors. In 1992, LLP developed the Improved accuracy from combined technology (IMPACT) method, which utilizes both magnetic DF and TOA data to locate strikes [18]. This method can employ information from TOA sensors, magnetic DF sensors and IMPACT sensors (which combine TOA and DF technology all in one sensor) [18]. The IMPACT sensors basically incorporated the measurement of arrival time in the magnetic DFs with the use of a GPS clock. The lightning locating principles behind magnetic DF and TOA technology were described in Section 2.3.1 and Section 2.3.2, respectively. Section 3.4.1 will discuss the IMPACT sensors and the algorithm behind the hybrid IMPACT method. The focus of this section is to describe the development of the NALDN in detail.

The first major upgrade to the NLDN began in 1994 and was completed in 1995. Global Atmospherics partnered with the EPRI to improve the NLDN performance in order to produce operational benefits targeted at the electric power industry [24]. The electric power industry required greater accuracy in lightning location and more comprehensive information in order to design more effective lightning protection for transmission and distribution systems. [24]. The main focus of the 1995 upgrade of the NLDN was to [24]:

- Report strokes as well as flashes

- Improve location accuracy

- Increase the percentage of lightning discharges detected

- Report peak current estimates for cloud-to-ground lightning strokes 
These goals were achieved through the IMPACT method and sensors, which combined magnetic DF and TOA technology. The original DF sensors used in the network were replaced with first generation IMPACT sensors. The upgraded NLDN contained 59 of the TOA sensors from the original ARSI national network (Lightning Positioning And Tracking System - Series III (LPATS-III) sensors) and 47 LLP IMPACT sensors [18]. All of the sensors used GPS timing for maximum arrival time accuracy. Because of the increase in the effective range of the sensors, the total number of NLDN sensors was reduced from over 130 to 106 in the 1995 upgrade, with typical sensor baselines values between 275 to $325 \mathrm{~km}$ [18]. The locations of these sensors across the U.S. is displayed in Figure 3.2.1.

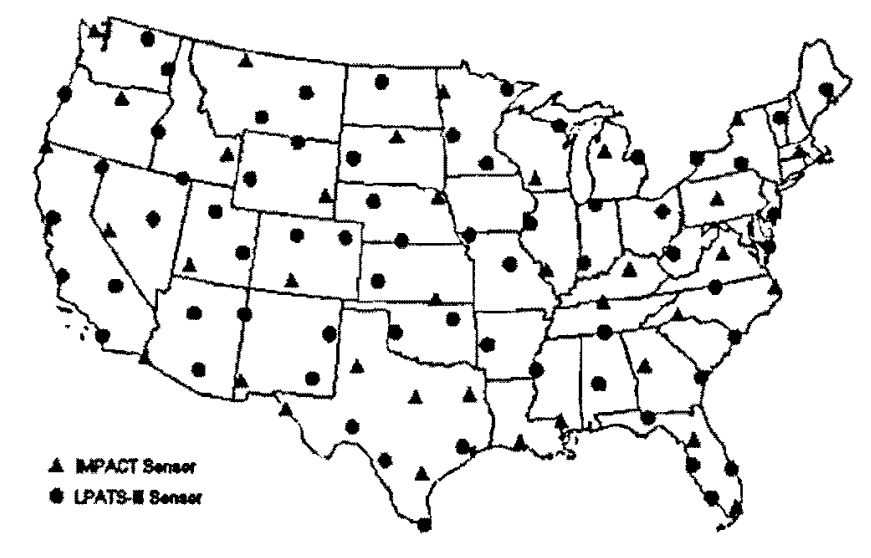

Figure 3.2.1: Locations of the sensors in the U.S. NLDN after the 1995 upgrade. (Adapted from [18].)

The IMPACT and LPATS-III sensors were both modified as part of the 1995 upgrade. The gain of the IMPACT sensor was increased, the trigger threshold was reduced, and the waveform width acceptance criteria was changed (narrower waveforms were accepted) to allow the detection of lower peak currents and more distant lightning [1]. In order to reduce undesirable cloud-to-cloud triggering, the LPATS-III sensors had their gains reduced and had waveform acceptance criteria implemented which was similar to the IMPACT sensors [1]. This resulted in both sensor types detecting cloud-to-ground flashes with similar sensi- 
tivity and discrimination [18]. In the early 1990s, several IMPACT sensors were tested and calibrated in the existing NLDN. The gain of the IMPACT sensors relative to the original NLDN magnetic DF sensors was determined [18]. After the upgrade, gain corrections were derived for the LPATS sensors to normalize their signal strengths to the values reported by the calibrated IMPACT sensors [18]. Therefore, the peak current estimates of the NLDN were not changed by the upgrade.

Before February 1998, lightning occurrence information in Canada was only available from manned surface stations and a few provincial networks with limited coverage [20]. The Ontario provincial network of LLP magnetic DF sensors in 1989 is shown in Figure 3.2.2.

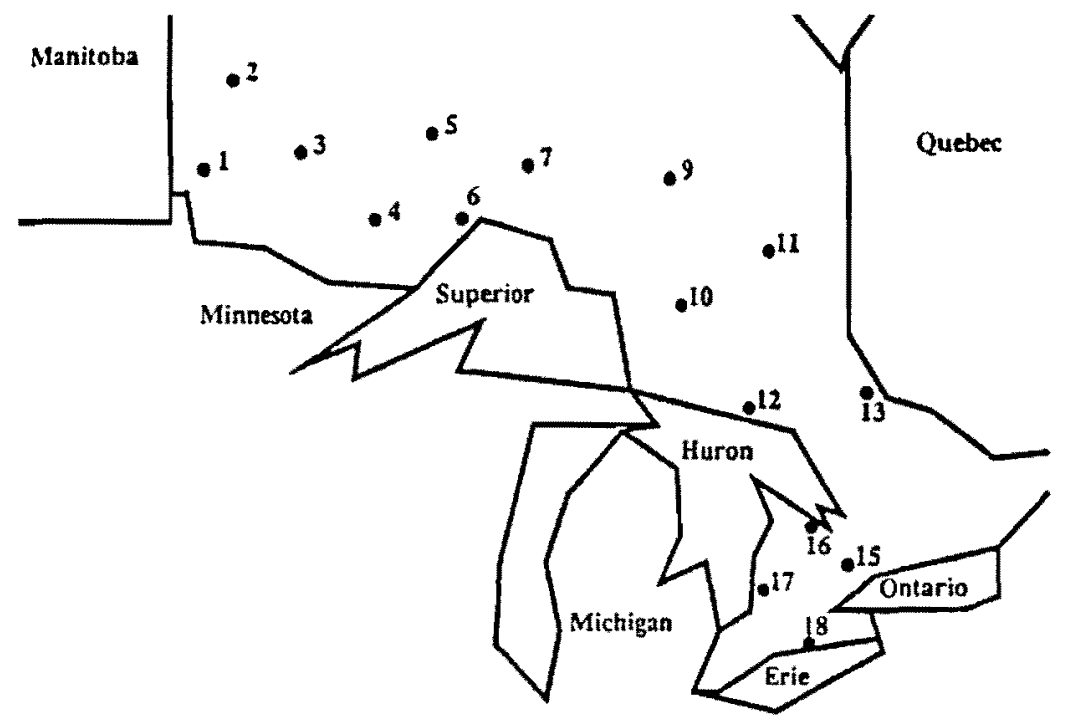

Figure 3.2.2: Location of the LLP magnetic DFs in the Ontario LLS in 1989. (Adapted from [13].)

According to [13], in 1989 Ontario had a provincial network consisting of 16 LLP magnetic DF sensors. Data from these sensors was transmitted to a position analyzer in Sault Ste. Marie, which stored the information on magnetic tapes.

In 1998, the Canadian Lightning Detection Network (CLDN) began its first year of operation. As previously mentioned, GAI supplied, installed and operated the CLDN for 
Environment Canada. The CLDN is composed of 81 sensors: 26 Improved Accuracy from Combined Technology - Enhanced Sensitivity (IMPACT-ES) sensors and 55 Lightning Position and Tracking System - Series IV (LPATS-IV) TOA sensors [25]. The NLDN combined and integrated with the CLDN, was dubbed the NALDN in [26]. Assuming a nominal detection range of $600 \mathrm{~km}$ for each sensor, the area covered by the NALDN is nearly 20 million $\mathrm{km}^{2}$, over latitudes ranging from $25^{\circ}$ to $67^{\circ}$ in the west and $25^{\circ}$ to $55^{\circ}$ in the east [25].

At the time of installation, the IMPACT-ES and LPATS-IV sensors were next-generation sensors compared to the old IMPACT and LPATS-III sensors used in the NLDN. These sensors have the capability of detecting and locating intercloud and intracloud discharges; however, because these sensors are separated by 300 to $500 \mathrm{~km}$, only around 1 to $4 \%$ of cloud discharges are located by the CLDN [25, 27]. The CLDN is depicted in Figure 3.2.3.

From late 2001 until 2003, the NLDN underwent another major upgrade. After more than two years of research and planning, the Board of Directors of Sankosha (GAI's parent company) approved the upgrade in July 2001 [28]. As noted in Section 3.1, in March 2002 Vaisala acquired GAI from the Sankosha corporation. The acquisition by Vaisala did not seem to slow down the implementation of the upgrade, which was completed in 2003 . It should be noted that the amount of sensors in the NLDN varied over time due to general maintenance and small upgrades. Just prior to the start of 2003 upgrade, the NLDN had 63 LPATS-III sensors and 43 IMPACT sensors [5].

The need to improve performance by using more advanced technology and the difficulty in maintaining aging equipment led to the second upgrade of the NLDN. The main goals of the 2003 NLDN upgrade were to $[5,24]$ :

- Improve network performance by using newer sensing technology

- Minimize maintenance and sensor downtime by replacing aging sensors and sensing station equipment 
- Build a stronger base for future improvements, including LF cloud-to-cloud lightning detection

- Provide enhanced detection efficiency (DE) and location accuracy on the boundary of the network

The 2003 upgrade of the NLDN consisted of the installation of 113 new third generation Improved Accuracy from Combined Technology - Enlanced Sensitivity and Performance (IMPACT-ESP) sensors at sensing stations throughout the continental United States [24]. These sensors replaced the original first generation IMPACT sensors, and the now outdated LPATS-III sensors which only used TOA technology. In order to optimize network geometry, several new sensor locations and relocations were implemented, as shown in Figure 3.2.3. The CLDN still contains many LPATS-IV sensors which only measure the TOA, however, the new IMPACT-ESP sensors from the upgraded NLDN provide coverage over most of Southern Ontario.

The following information regarding the new sensors used in the NLDN is summarized from [5]. Like earlier versions of the IMPACT sensor, the the IMPACT-ESP sensor detects both electric and magnetic fields, and provides the arrival time and azimuth for each discharge. The IMPACT-ESP sensor has improved analog front end circuitry, a higher speed processor and configurable waveform criteria. According to [5], the improved analog front end reduces noise, allowing for better detection of small amplitude signals. This improves detection efficiency, particularly for small peak current events. The higher speed processor reduces the significant dead-time after an event, which is due to the time required to process and report the event. Impact-ESP sensors have a dead-time on the order of one millisecond, which is especially important for detecting cloud-to-cloud discharges, which are typically much smaller in amplitude than return strokes but occur more frequently. The configurable waveform and noise-rejection criteria allow the IMPACT-ESP sensor to reject or accept different waveform shapes (as a function of angle and signal strength), and to categorize the 


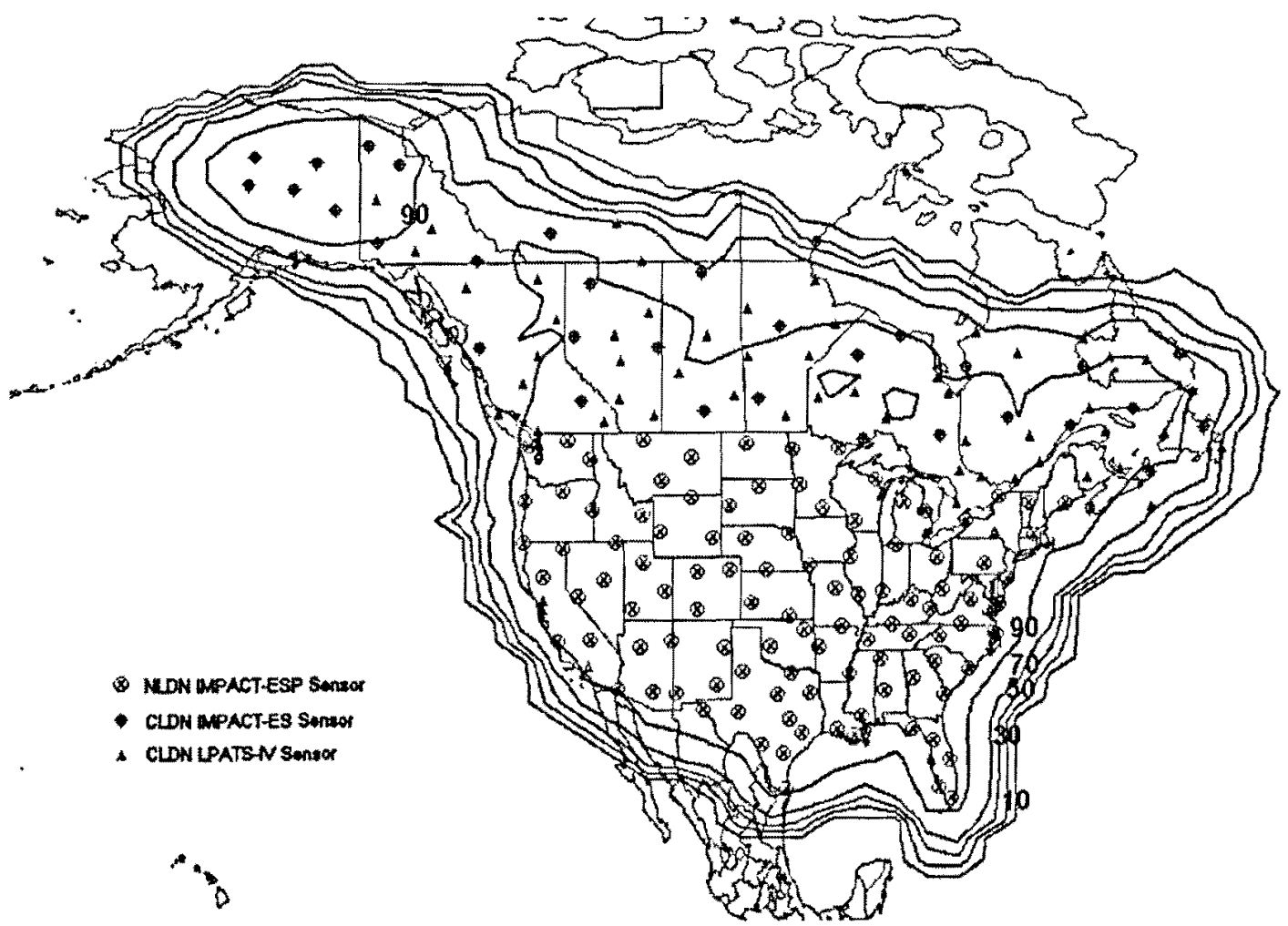

Figure 3.2.3: The NALDN after the 2003 NLDN upgrade. The projected flash detection efficiency is displayed. (Adapted from [5].)

event as cloud-to-ground or cloud-to-cloud based on a set of rules that can be modified as needed. Because of these improvements, the NLDN should be able to detect a larger fraction of cloud-to-cloud discharges, and a greater amount of return strokes from cloud-to-ground flashes. The actual performance of the NALDN is discussed later on in Section 3.6

\subsection{Operations and Communications}

The NALDN estimates the following lightning information $[24,29]$ :

- time of stroke

- location of stroke 
- polarity of stroke

- peak current of stroke

- confidence ellipse

The confidence ellipses mentioned above are available from Vaisala and reflect a percentage of certainty that a lightning event occurred inside the ellipse. These ellipses are used as a method to determine the location accuracy of the NALDN, and are thoroughly discussed in Section 3.5.1.

A graphical representation of the real-time NALDN operation and data flow is shown in Figure 3.3.1. As previously mentioned, the CLDN is owned by Environment Canada and operated under contract by Vaisala, with all data being processed at the Network Control Center (NCC) in Tucson, Arizona. From there, the lightning discharge information is transmitted to Environment Canada's weather centers. The real-time NALDN data flow depicted in Figure 3.3.1 works as follows [5, 18, 29, 30]:

1. Sensors detect lightning and transmit the data to a satellite.

2. The satellite relays the information to earth stations.

3. The downlink site forwards data via an internet link to the NCC in Tucson, Arizona.

4. Data from the remote sensors are processed in the NCC to provide the time, location, polarity and the current peak of each cloud-to-ground lightning flash in real-time.

5. This processed information is then sent back out the communications network for satellite broadcast dissemination to real-time users.

6. Lightning data appears on user's displays across the country within 30 to $40 \mathrm{~s}$ of occurrence.

The following explanation of the NALDN operation is summarized from [5] and [18]. As mentioned above, all of the above processes take place within 30 to $40 \mathrm{~s}$ of a lightning 


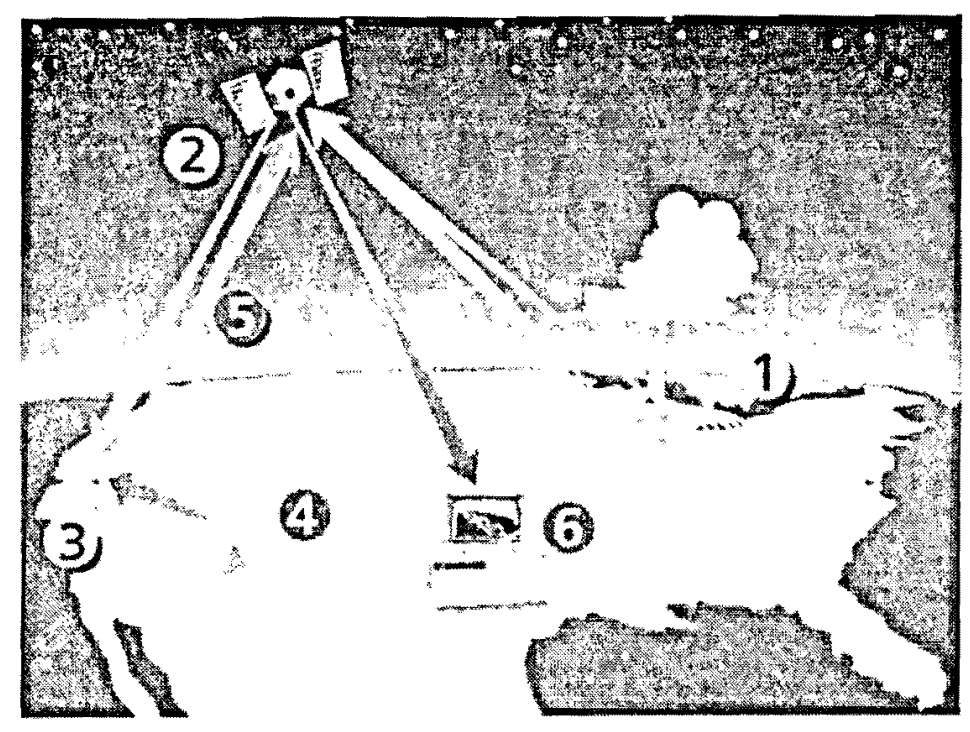

Figure 3.3.1: Real-time data flow in the NALDN [18]

discharge. This delay consists of a fixed $30 \mathrm{~s}$ hold time and a variable processing and communications delay. Cloud-to-ground flash information with a $0.1 \mathrm{~s}$ time resolution is immediately distributed via the satellite broadcast link, while higher resolution flash and stroke data (including confidence ellipses) are available through other communications links. The lightning data are also reprocessed within a few days of the real-time acquisition and archived in a permanent database for users who do not require real-time data.

As explained in [5] and [18], the real-time data are subject to two sources of error that do not affect the reprocessed data. These sources are sensor calibration errors and communications delays. Calibration errors consist of magnetic DF site errors and peak field amplitude calibration errors, as explained in Section 2.3 .1 and Section 3.4.4 respectively. Recently with the 2003 upgrade of the NLDN, the procedure for initial calibration and maintenance of the magnetic DF site errors has been improved. Site errors are continuously monitored and quickly corrected. According to [5], through the use of new software, site error corrections can be verified and applied within a few hours of data collection.

Sensor communication delays may result from rain fade or data congestion during periods 


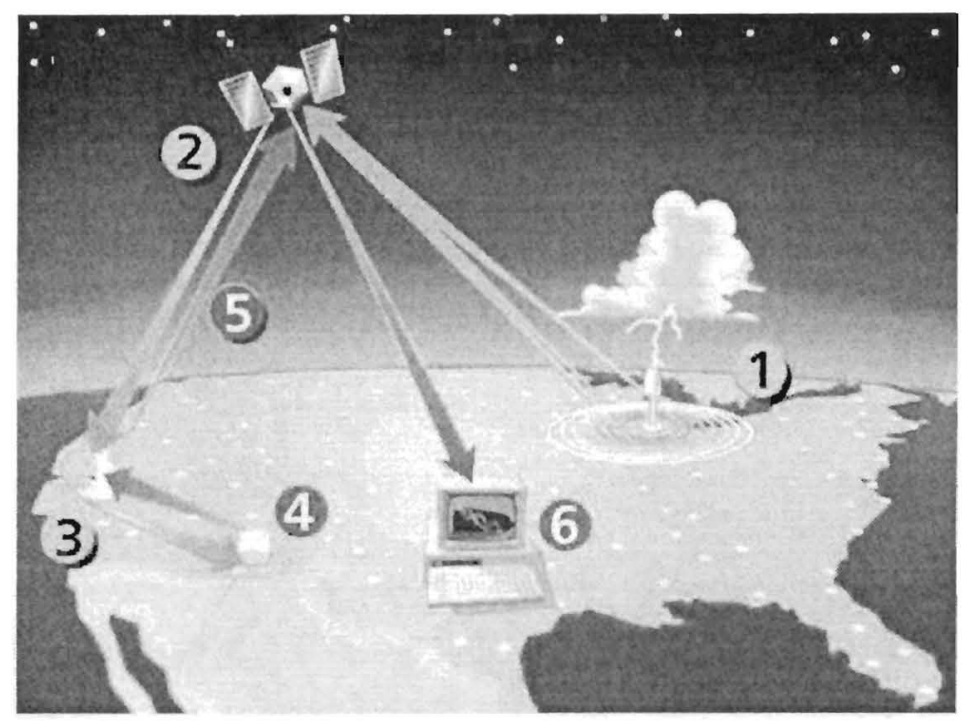

Figure 3.3.1: Real-time data flow in the NALDN [18]

discharge. This delay consists of a fixed $30 \mathrm{~s}$ hold time and a variable processing and communications delay. Cloud-to-ground flash information with a $0.1 \mathrm{~s}$ time resolution is immediately distributed via the satellite broadcast link, while higher resolution flash and stroke data (including confidence ellipses) are available through other communications links. The lightning data are also reprocessed within a few days of the real-time acquisition and archived in a permanent database for users who do not require real-time data.

As explained in [5] and [18], the real-time data are subject to two sources of error that do not affect the reprocessed data. These sources are sensor calibration errors and communications delays. Calibration errors consist of magnetic DF site errors and peak field amplitude calibration errors, as explained in Section 2.3.1 and Section 3.4.4 respectively. Recently with the 2003 upgrade of the NLDN, the procedure for initial calibration and maintenance of the magnetic DF site errors has been improved. Site errors are continuously monitored and quickly corrected. According to [5], through the use of new software, site error corrections can be verified and applied within a few hours of data collection.

Sensor communication delays may result from rain fade or data congestion during periods 
of high data rates. Data congestion occurs if the lightning rate over the entire network exceeds a certain amount of flashes per hour; leading to data arriving too late at the NCC to be used in real-time processing. In these situations, the reprocessed data usually contained 2 to $5 \%$ more strokes than the real-time data. However, according to [5], the links between the downlink site and the NCC have been recently been upgraded in order to minimize this issue. Although weather related communications issues such as rain fade can still lead to late data and possibly missed events in the real-time datastream, data congestion issues have practically been eliminated from the network. Reprocessed data still contains a larger number of strokes in the summer months though, now on the order of 1 to $2 \%$.

The entire NALDN and every sensor in the network are continuously monitored to ensure data quality and proper operation. According to [4] and [29], the NALDN (including both the NLDN and CLDN) has a $99.7 \%$ or better uptime. To maintain this reliability and performance, the following measures are implemented [24]:

- System-wide Redundancy

- Two separate satellites and acquisition hubs acquire data from two evenly distributed sensor configurations. If one satellite, its hub, or its hub communication link fails, the sensor set reporting to the remaining satellite and hub provides sufficient coverage.

- Private carrier-grade communications system of direct links with back-up links in the event of a primary circuit failure.

- Two central processors operating independently with automatic fail-over for uninterrupted analysis of incoming sensor data and lightning solution output.

- Back-up network control center is available at the State University of New York at Albany.

\section{- Quality Control}

- NCC operators review regional network performance hourly using a graphical statistical analysis tool displayed by the central processor. 
- Comprehensive statistical reports on regional network performance and on individual sensor performance are generated by the central processor and reviewed daily by staff scientists.

- Failure notification of any individual sensor occurs within one minute.

- On-call repair and maintenance staff are dispatched to sensor sites usually within 48 hours of sensor failure and regional technicians can provide even more rapid response when needed.

- Uptime and Availability

- 99.9789\% average uptime for data acquisition from sensors (2003).

- $99.9928 \%$ average availability of data broadcast to users via satellite (2003).

- 99.9413\% average availability of Internet services (2003).

\section{- Mission Critical Operations}

- NCC is staffed 24 hours a day, 7 days a week, 365 days a year.

- Controlled access to NCC.

- Uninterrupted power supply with gas generator for sustainable power source to the NCC.

- Disaster recovery procedure in place.

The NALDN data is available to customers through various software packages and Internet services. These application tools are specialized for real-time lightning tracking and warning, or for the analysis of past lightning activity [24]. Real-time data is delivered by satellite broadcast or Internet, while historic lightning data can be delivered by cdrom, fax, or the Internet [24]. As shown on Vaisala's website [31], the following Vaisala software packages or services are available for both Canadian and American use with NALDN data: Vaisala LTraX Real-time Lightning Tracking Software, Vaisala FALLS Fault Analysis and Lightning Location System, Vaisala FaultFinder, Vaisala STRIKEfax Fax Lightning Verification Report, and Vaisala STRIKEnet Lightning Verification Report. It is interesting to 
note that Internet lightning notification from Vaisala using "Vaisala Lightning Observer 2.0" and "Lightning Notification for Groups" is only available in the U.S. [32]. However, Environment Canada has recently begun to offer a similar service in Canada named "e-Flash" [33]. All of these services are used by many industries for a variety of applications which are discussed in detail in Section 3.7.

\subsection{Location and Stroke Processing Algorithms}

\subsubsection{The IMPACT Location Method}

As previously mentioned, the IMPACT method can use information from any combination of magnetic DF sensors, TOA sensors and IMPACT sensors (which combine TOA and DF technology all in one sensor) [18]. Historically, the TOA sensors worked by measuring the time-of-arrival difference of an electric field signal from lightning between different sensors, producing a hyperbolic curve as explained in Section 2.3.2. As explained in [10], in the IMPACT method the magnetic direction finding provides the azimuth information, while the absolute arrival time now provides the range information.

The IMPACT sensors detect and measure the magnetic field with 2 crossed loop antennas for the determination of a direction vector which points towards the stroke. These sensors will also use a plate antenna to detect the electric field and determine a TOA range circle (with measurement of arrival time done through the use of a GPS clock). The LPATS-IV sensors which are still in use in the CLDN will detect the electric field using a whip antenna and also create a TOA range circle (with measurement of arrival time done through the use of a GPS clock).

These measurements generate the latitude, longitude, and discharge time of a stroke. Therefore, the IMPACT method has redundant information allowing for an optimized esti- 
mate of a strike location, even when only two sensors provide timing and angle information. For example, if a strike happens on the baseline between two sensors as shown in Figure 3.4.1, the IMPACT sensors will provide two direction vectors from the azimuth information and two TOA range circles.

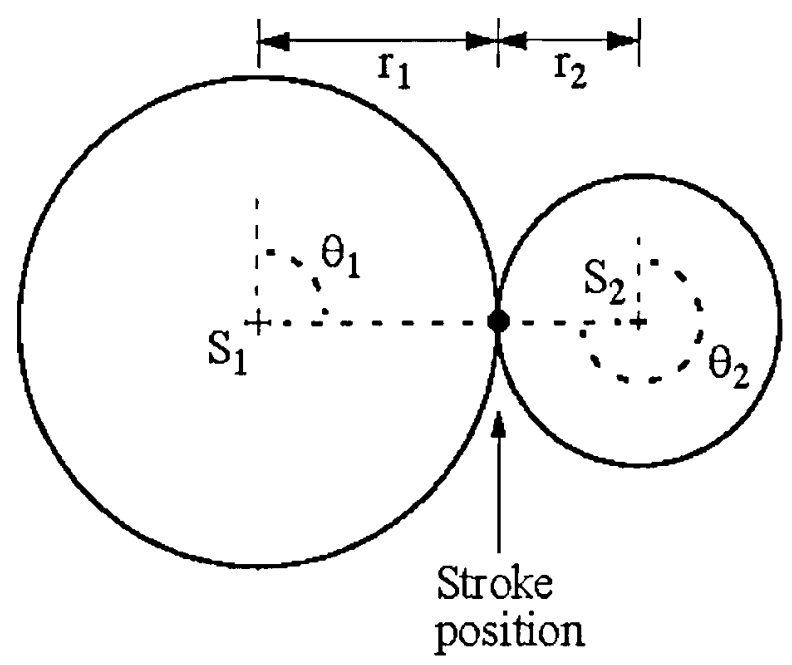

Figure 3.4.1: An event occurring on the baseline between two IMPACT sensors (Adapted from [34].)

Figure 3.4.2 shows a typical lightning stroke in Florida that was detected by five sensors (three IMPACT and two LPATS-III sensors) in the NLDN before the 2003 upgrade [10]. Once again, the DF measurements produced the two direction vectors (straight lines) in the figure, while the TOA measurements produced the five range circles centered around each sensor. Clearly there is an abundance of redundant information from the five sensors, which all seem to have located the stroke approximately at the same location. The two magnetic DF vectors seem to converge at the same location as the five TOA range circles.

It should be noted that all of the sensors in the NLDN provide both TOA and magnetic DF information since the 2003 upgrade, and therefore only two sensors are required to locate a discharge as shown in Figure 3.4.1. Prior to this upgrade, an average of three to four sensors were required to compute a location, since $60 \%$ of the sensors in the network 


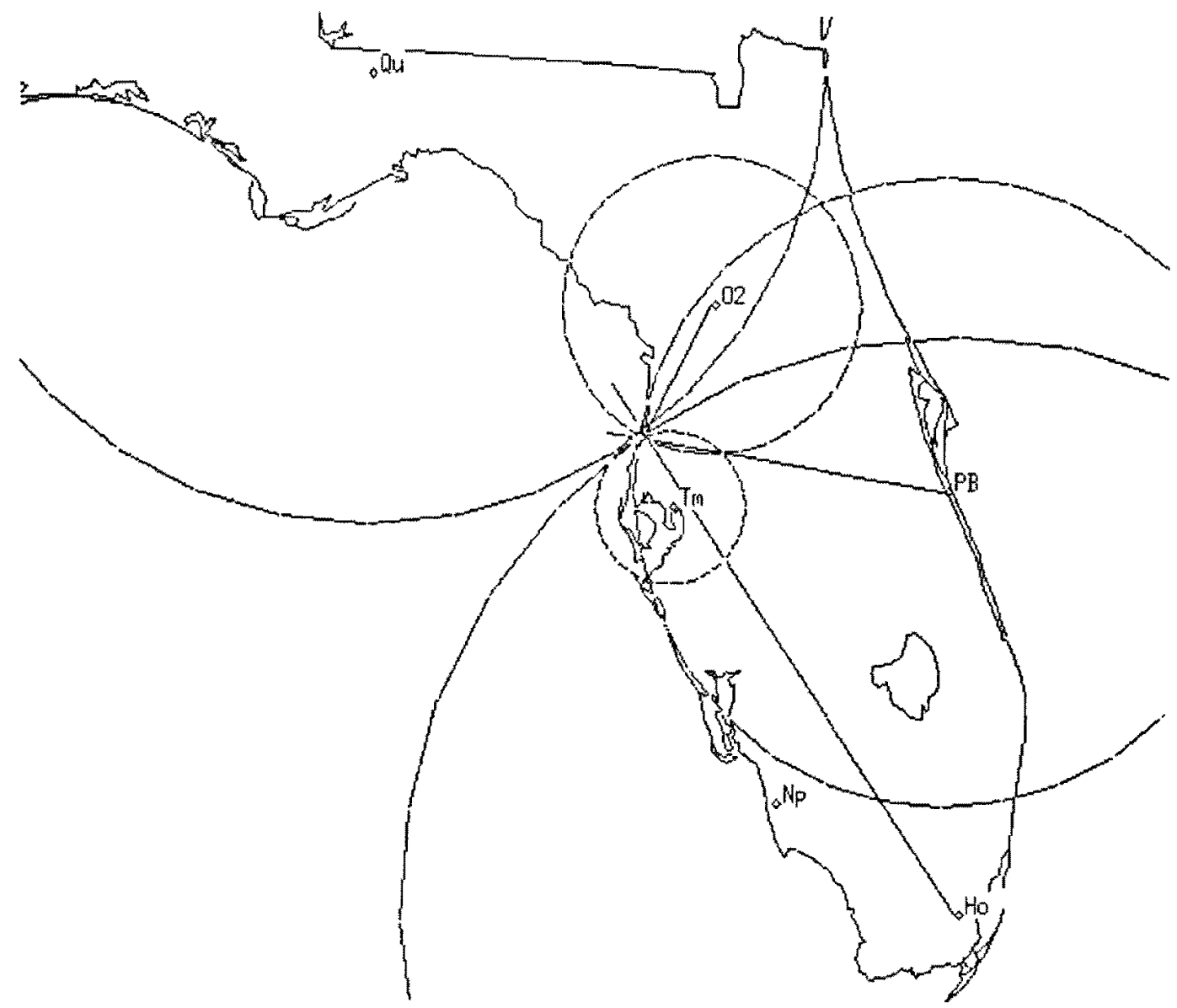

Figure 3.4.2: Example of the IMPACT algorithm using three LPATS TOA sensors and two IMPACT sensors. (Adapted from [10].)

only provided TOA information [5]. The CLDN still contains many LPATS-IV sensors which only measure the TOA, however, the new IMPACT-ESP sensors from the upgraded NLDN provide coverage over most of Southern Ontario as shown in Figure 3.2.3.

The following description of the IMPACT algorithm is taken from [1]. The IMPACT algorithm works by using the data from the NALDN sensors to produce an optimum lightning location using a least squares minimization technique, where the following $\chi^{2}$ function is minimized: 


$$
\chi^{2}=\sum_{i=1}^{N 1}\left(\frac{\theta_{m i}-\theta_{i}}{\sigma_{\theta_{i}}}\right)^{2}+\sum_{i=1}^{N 2}\left(\frac{t_{m i}-t_{0}}{\sigma_{t i}}\right)^{2}+\sum_{i=1}^{N 2}\left(\frac{E_{m i}-E_{i}}{\sigma_{E_{i}}}\right)^{2}
$$

where $t_{0}$ is the unknown time of the event, $t_{m i}$ is the measured time-of-arrival at the $i$ th station minus the time required for the signal to propagate from the source to the station, $\sigma_{t i}$ is the expected error in the time measurement, $\theta_{i}$ and $E_{i}$ are the unknown azimuth and electric field peak values, $\theta_{m i}$ and $E_{m i}$ are the azimuth and electric field peak measured at the $i$ th station, $\sigma_{\theta_{i}}$ is the expected azimuth measurement error, and $\sigma_{E_{i}}$ is the expected measurement error in the peak electric field. The summation in the first term of Equation 3.4 .1 is over the total number (N1) of IMPACT sensors reporting azimuth angle $\theta_{m i}$, while the summation in the second and third terms is over the total amount (N2) of both IMPACT and LPATS sensors reporting time $t_{m i}$ and electric field peak $E_{m i}$. Basically the optimum location (latitude, longitude) and onset time at the source are determined by finding the position on an oblate spheroidal earth that makes the unconstrained $\chi^{2}$ error function a minimum; the value of this function at the minimum describes the overall accuracy of the location [35].

The following is summarized from [1] and [18]. Apparently, only the first two terms on the right-hand side of Equation 3.4.1 are routinely used for locating a lightning strike. The relative contribution of timing and angle errors to the total $\chi^{2}$ value is determined by taking into account their individual measurement errors, expressed in the form of standard deviations. The standard deviation in the measured TOA is assumed to be $\sigma_{t i}=1.5 \mu s$ and the standard deviation in angle is assumed to be $\sigma_{\theta_{i}}=0.9^{\circ}$. These errors account for the variable terrain effects encountered, such as propagation differences between the central Great Plains and the Rocky Mountains, and other such random errors. Increasing the angle standard deviation to $1.5^{\circ}$ and the time standard deviation to $2.0 \mu \mathrm{s}$ has little effect on the estimated location accuracy. The standard deviation in the peak electric field $\sigma_{E_{i}}$ is assumed to be $10 \%$ of $E_{m i}$. The performance and location accuracy of the NALDN is discussed later 
on in Section 3.6.

\subsubsection{Flash Multiplicity and Polarity}

The following information is summarized from [1] and [18]. In addition to locating individual strokes, the NALDN also groups the strokes into flashes and determines polarity using the measured electric field peak. Prior to the 1995 upgrade of the NLDN, the multiplicity (number of strokes) of a flash was found by accumulating a count of all the strokes that occurred within $2.5^{\circ}$ of the first stroke for a period of $1 \mathrm{~s}$ after the first stroke, with the flash multiplicity being the largest number of strokes detected by any magnetic DF. This tended to overestimate the multiplicity as shown in Figure 3.4.3.

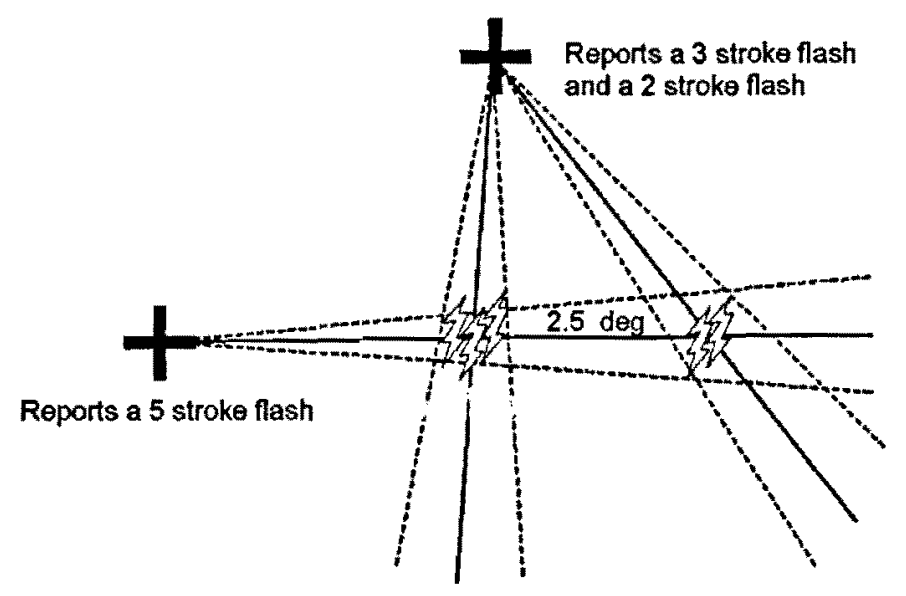

Figure 3.4.3: Angle-based flash grouping algorithm, illustrating how multiplicity can be overestimated. (Adapted from [18].)

In the current NALDN, strokes are added to a flash for a period of $1 \mathrm{~s}$ after the first stroke, if the additional strokes are within $10 \mathrm{~km}$ of the first stroke and the time interval from the previous stroke is less than $500 \mathrm{~ms}$. If a stroke happens to qualify as a part of more than just one flash, it is grouped into the flash with the closest first stroke. Furthermore, a stroke is included in a flash if it is located within 10 to $50 \mathrm{~km}$ of the first stroke and the 
$50 \%$ confidence ellipses of the strokes overlap. Section 3.5.1 discusses how confidence ellipses are determined for the NALDN. The maximum multiplicity for a flash is 15 strokes, with any subsequent strokes being treated as part of a new flash. This may underestimate the multiplicity of a flash because according to [1], the percentage of flashes that have more than 15 strokes has been observed to be $2.6 \%$ in Florida and $4.8 \%$ in New Mexico. Furthermore, many small subsequent strokes may fall below the trigger threshold and not be detected. Figure 3.4.4 displays the current stroke grouping algorithm.

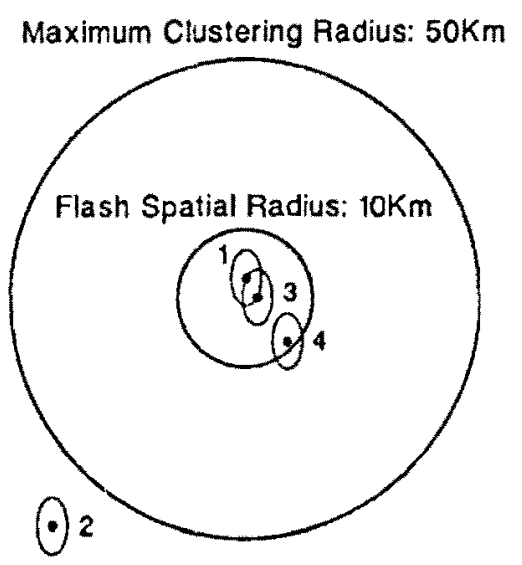

Figure 3.4.4: Location-based flash grouping algorithm. Strokes 1, 3, and 4 constitute one flash; stroke 2 is regarded as a separate flash. (Adapted from [18].)

It should be noted that the reported NALDN flash location is the location of the first stroke (number 1 in Figure 3.4.4), and the peak current estimate for a flash is also from the first stroke. If a subsequent stroke has the opposite polarity from the first stroke, it is still counted in the flash; however, the reported polarity of the flash is that of the first stroke. Obviously there is also individual location, peak current, time and polarity information for each stroke detected within a flash. 


\subsubsection{Flash and Stroke Times}

The following is summarized from [35]. It should be briefly mentioned that the flash time reported by the NALDN is the estimated time of the onset of the first return stroke in the discharge. All sensors in the NALDN use GPS timing. The time of a stroke is derived in the optimization algorithm of Equation 3.4.1, using the measured arrival times at each sensor after subtracting the time required for the field to propagate from the source to the sensors. According to [35], because the stroke time is a free parameter in Equation 3.4.1 and the source position is uncertain to within a few hundred meters ( $500 \mathrm{~m}$ median accuracy), the onset time should be accurate to approximately $5 \mu \mathrm{s}$. This time resolution of $5 \mu \mathrm{s}$ is more than adequate to resolve separate return strokes within a flash.

\subsubsection{Peak Current Estimation}

The following information is summarized from [1] and [18]. The NALDN provides an estimate of the stroke peak current using measurements of the peak field signal strength. The relationship between peak field and peak current assumes that the simple transmission line model (TLM) from [1] is valid for the peak field. The first step in the estimation

procedure is to take propagation effects into account and produce a range-normalized value of the signal strength (RNSS) in LLP units for each reporting sensor, using the following signal propagation model $[13,18]$ :

$$
R N S S=C * S S *\left(\frac{r}{I}\right)^{p} \exp \left(\frac{r-I}{A}\right)
$$

where $S S$ is the signal strength reported by the sensor, $r$ is range in kilometers, $I$ is the normalization range which is set to $100 \mathrm{~km}, p$ is an attenuation exponent, $A$ is the e-folding length for attenuation (space constant), $C$ is a constant, and RNSS is the range-normalized signal strength in LLP units. 
After the 1995 upgrade of the NLDN, the attenuation exponent used in the NLDN was $p=1.13$; it was determined empirically in Florida by assuming that $A$ was infinite [36]. The value of $A$ in the NLDN was $10^{5} \mathrm{~km}$. In [13], values of $A$ between 600 and $1000 \mathrm{~km}$ were found in Ontario using the exponential form of the model with $p=1$. The values of RNSS for all reporting sensors within $625 \mathrm{~km}$ (to avoid polarity reversals due to ionospheric skip) are averaged, and then the stroke-average RNSS is converted to an estimate of peak current. Prior to the 1995 NLDN upgrade, the peak current conversion was done with a linear regression equation that was derived in [37]. For $p=1.13$, this equation is:

$$
I_{p e a k}=5.2+0.148 R N S S
$$

where $I_{\text {peak }}$ is in kiloamperes. The nonzero intercept in Equation 3.4 .3 may reflect the fact that the NLDN did not detect many strokes with peak currents below $5 \mathrm{kA}$. However, the sensors after the 1995 NLDN upgrade did detect smaller signal strengths making Equation 3.4.3 unsuitable. Therefore, the intercept was modified to zero and the linear regression was recalculated using the data from [37], resulting in:

$$
I_{p e a k}=0.185 R N S S
$$

where $I_{p e a k}$ is in kiloamperes. This formula was derived by comparing the calibrated RNSS value to peak current measurements in rocket-triggered lightning.

The following is summarized from [5]. After the 2003 upgrade of the NLDN, the increased sensitivity of the IMPACT-ESP sensors prompted the reexamination of the above model. The space constant which was $A=10^{5} \mathrm{~km}$ and the attenuation exponent which was $p=1.13$ were sufficient for lightning events that were located within $400 \mathrm{~km}$ of a sensor, however, they underestimated the propagation losses of events that were more distant. Subsequently, it was found that the exponential form of the model ( $p=1$ and $A$ set to a smaller value) produces better estimates of propagation losses. This is similar to the results found in [13] 
for Ontario, where values of $A$ between 600 and $1000 \mathrm{~km}$ were found using the exponential form of the model with $p=1$. Similarly, in the recently upgraded NLDN, it was found in [5] that changing the model parameters to $p=1$ and $A=1000 \mathrm{~km}$ reduced the random error in the model by $11 \%$. The performance and accuracy of the peak current estimation in the NALDN is discussed later on in Section 3.6.4.

\subsection{Measures of Performance: Location Accuracy and Detection Efficiency}

The performance of the NALDN is measured in two ways: location accuracy and detection efficiency (DE). The DE of the network is the fraction of actual cloud-to-ground strokes or flashes that are detected and reported by the network. In order to evaluate and predict the performance of different sensor configurations in a lightning location network, GAI developed the following location accuracy and detection efficiency models. These models have proven to be very useful for the design and evaluation of the NALDN.

\subsubsection{Location Accuracy Model}

The following explanation of how the NALDN evaluates location accuracy is summarized from [1] and [18]. The average location error in the NALDN is defined as the serni-major axis of the $50 \%$ confidence ellipse surrounding the optimum location obtained from minimizing $\chi^{2}$ in Equation 3.4.1. Assuming that there are no significant systematic errors and that random angle and time errors are Gaussian, there is a $50 \%$ chance that the located stroke is within the $50 \%$ confidence ellipse, and a $50 \%$ chance that it is outside of the ellipse. Confidence regions are elliptical when errors are Gaussian. These error ellipses are used as a measure of location accuracy in NALDN data and are available to consumers. 


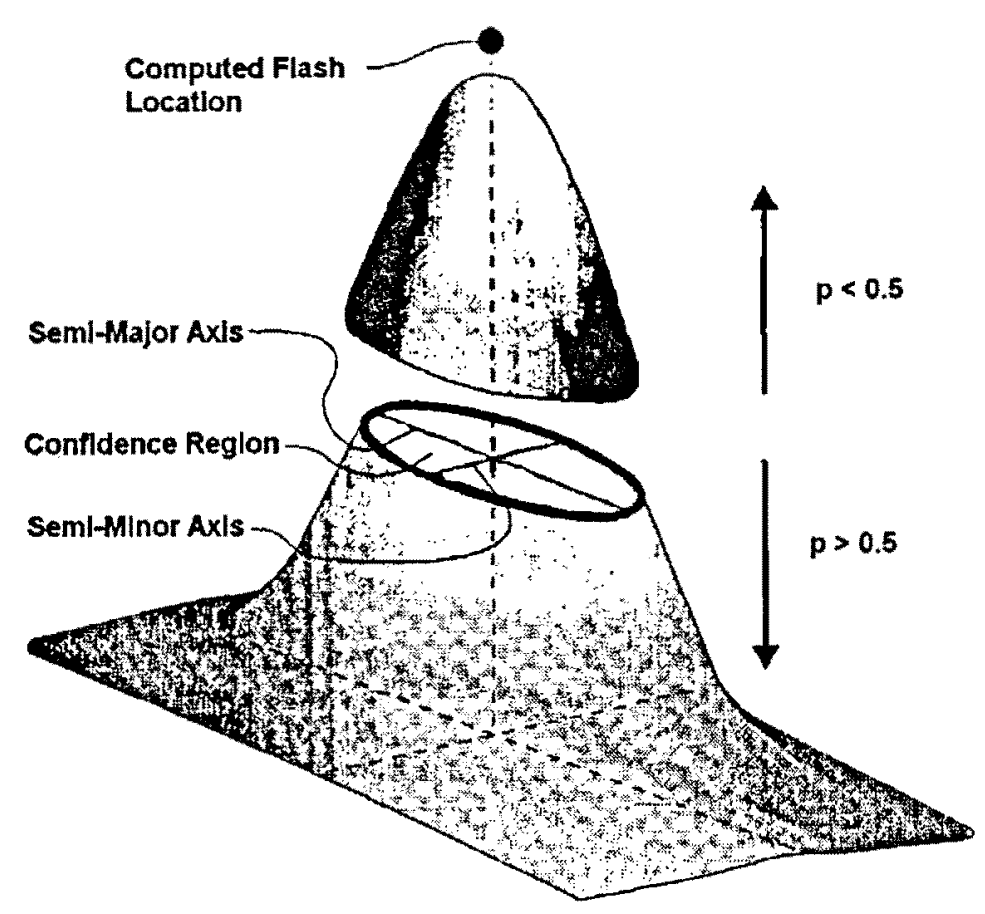

Figure 3.5.1: Two-dimensional Gaussian distribution of location errors showing the estimated stroke location at the most probable point (the peak). The $50 \%$ error ellipse is derived by cutting the distribution at a probability level of 0.5 . (Adapted from [18]).

Figure 3.5.1 shows a two-dimensional Gaussian distribution of location errors from which an error ellipse is derived. The optimum stroke location, which is obtained from minimizing $\chi^{2}$ in Equation 3.4.1, is at the most probable point (the peak) of the error distribution. As shown in Figure 3.5.1, at any probability level $p$, there is an elliptical confidence region in which there is a probability $p$ of finding the true stroke location. The reference probability level is 0.5 in the NALDN, so that the error ellipse expresses the median location accuracy.

The two-dimensional Gaussian distribution of errors in latitude and longitude is based on the assumption that the random errors in time and angle measurements are uncorrelated and approximately Gaussian. This assumption is valid after site errors are corrected, and if propagation-based timing errors are small. Overall errors tend to be Gaussian when a large number of sensors are used, so even if the assumption is not completely valid, it is still 
appropriate. If the assumptions are invalid, then a large $\chi^{2}$ value would result in Equation

\section{4 .1 .}

The location of a stroke relative to the sensors will determine the shape of an error ellipse. For instance, when a stroke is outside the network and the distance from the nearest sensor is several times the sensor baseline length, the ellipse is very elongated and points in the direction of the sensors. Conversely, when a stroke is in the middle of several sensors, the error ellipse is small and nearly circular.

In practice, the location accuracy model computes the semi-major axis of the error ellipse at each point on a 50-by-50 grid over the network coverage area. The locations, types of sensors, and the average angle and timing errors are specified into the model. As shown in Figure 3.6.1 and Figure 3.6.3, the model output is a contour map of the semi-major axis. Vaisala's STRIKEnet also offers the option of viewing $99 \%$ confidence ellipses on a map, as shown in Figure 3.5.2 [38]. These ellipses indicate a 99\% probability that the recorded lightning discharge is within the bounds of the ellipse.

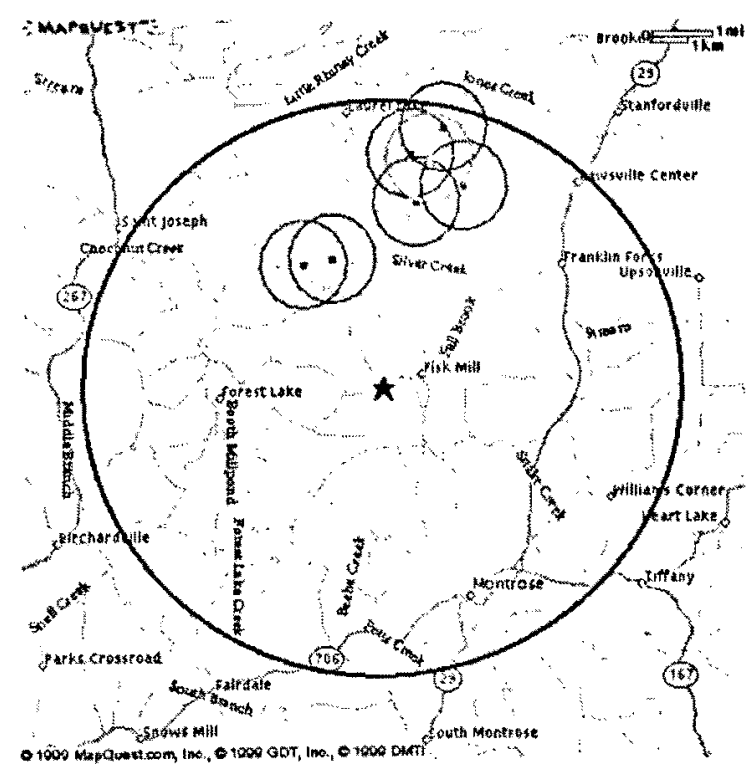

Figure 3.5.2: $99 \%$ confidence ellipses for all strikes detected within the search area using data provided by the NALDN. (Adapted from [39]). 


\subsubsection{Detection Efficiency Model}

The following explanation of how the NALDN evaluates detection efficiency was taken from [18]. The detection efficiency (DE) model developed by GAI computes estimates of the $\mathrm{DE}$ on a 50 -by-50 grid over the region of network coverage. The model generates specific values of peak current at each grid point, and computes the signal strength that should arrive at each sensor using the signal propagation model discussed in Section 3.4.4. The DE model then uses a look-up table that relates the computed signal strength at each sensor to that sensor's DE, producing a probability that the stroke will be detected by that sensor. The look-up table contains the response of each type of lightning sensor as a function of the signal strength. The simplest response for each sensor would be a probability of 1 for all events above the threshold, and a probability of 0 for all events below. However, realistic tables have been derived for all the sensors, using the measured responses from tens of thousands of lightning events. The look-up tabie values increase from zero probability at the threshold, to a maximum probability (which is less than one) at around two to three times threshold.

Assuming that the sensor probabilities are independent, the total probabilities are computed for all the combinations of sensors that report a discharge. This yields a total probability for any combination of sensors reporting a strike, which is simply the product of the probabilities of detection (and non-detection) for each sensor. This process is repeated over the entire range of peak currents for each grid point, to produce an overall estimate of the network DE. The model output is a contoured map of "percent detected," as shown in Figure 3.6.7 and Figure 3.6.8. 


\subsection{Comparison of Past and Present Performance}

As previously mentioned, the NALDN provides the time, location, polarity, peak current and confidence ellipse of each detected stroke. This section will compare the past and present performance of the NALDN by individually examining its location accuracy, flash detection efficiency (DE), stroke detection efficieny (DE) and peak current estimation. This is followed by a brief discussion on misclassified events and a summary of the overall performance of the NALDN.

\subsubsection{Location Accuracy}

As previously stated, in the early 1990 s the NLDN consisted only of magnetic DF sensors. Prior to 1992, GDS estimated that the average location accuracy of the NLDN varied from 8 to $16 \mathrm{~km}$ [18]. This estimate was based on observations, and the assumption that there were site errors which caused angle errors of 1 to $3^{\circ}$ in the magnetic DF sensors [18]. As discussed in [40], a study performed in 1990 (prior to the correction of site errors) confirmed these estimates. In early 1992, GDS calibrated the sensors to correct for site errors and determined that the average location accuracy was 2 to $4 \mathrm{~km}$ in the vicinity of the NASA Kennedy Space Center in Florida, which was in agreement with the estimated accuracy for that region. [18].

After the 1995 upgrade, the estimated contours of constant median accuracy (in kilometers) of the NLDN can be seen in Figure 3.6.1. These contours were determined using the location accuracy model which was described in Section 3.5.1. The model predicts that the majority of the U.S. will have a median location accuracy of $500 \mathrm{~m}$. According to the model, the NLDN was also able to locate lightning strikes in Southern Ontario with a median location accuracy of $500 \mathrm{~m}$, allowing the NLDN to also collect and archive data from this region. Indeed, Vaisala confirms that there is lightning data available for Southern Canada 
and also Northern Mexico beginning in 1995, stating that: "Due to its southern positioning most of the province of Ontario is covered by NLDN sensors" [41]. It is also interesting to note that the location accuracy and DE fall off near the edges of the network. The addition of the CLDN in 1998 eliminated these limitations on the northern border of the NLDN, but the coastal and southern borders continued to have location accuracy and DE limitations.

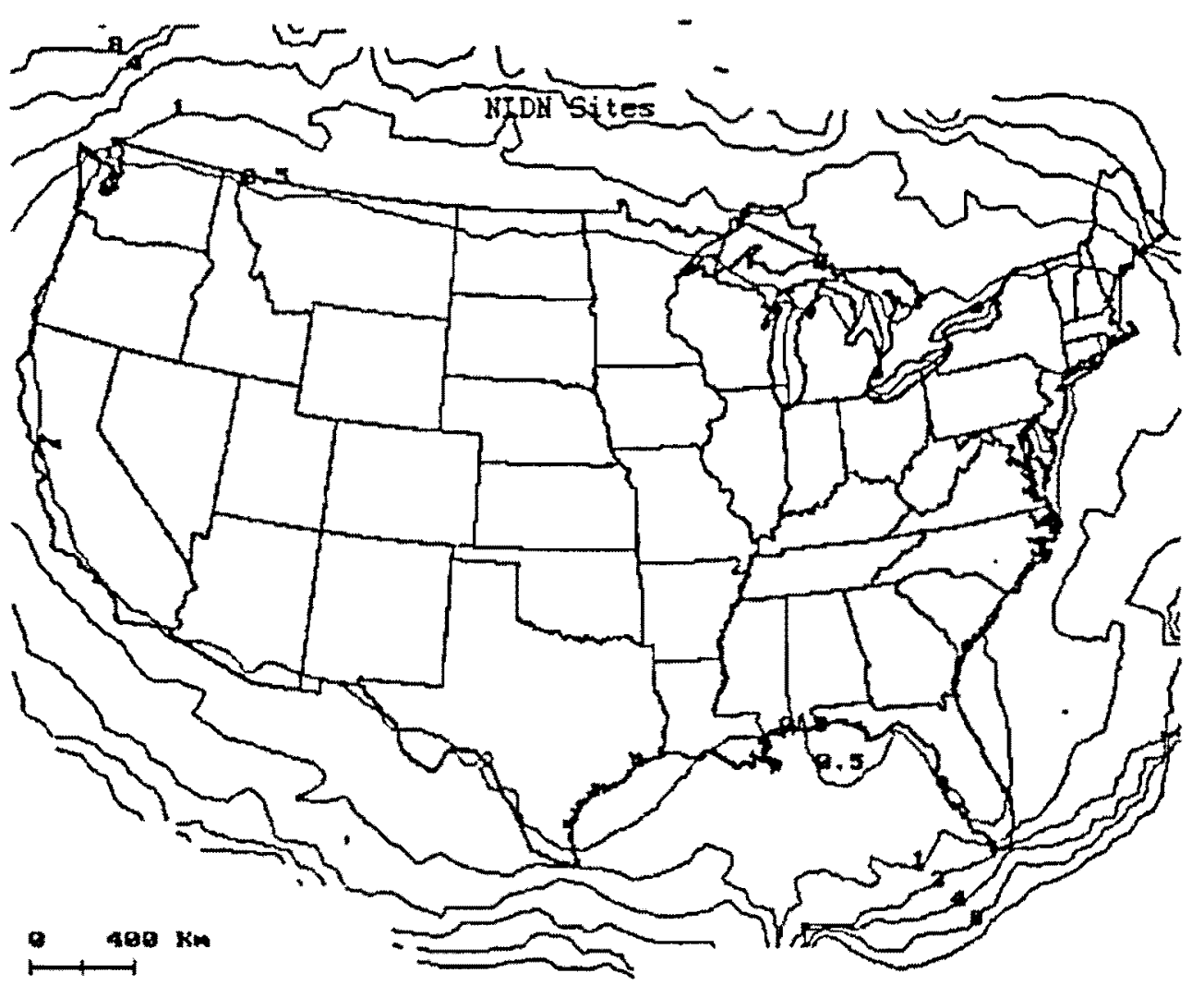

Figure 3.6.1: Projected semi-major axis of the error ellipse for the NLDN after the 1995 upgrade. Contour labels give the value in kilometers and represent the median ( $50 \%$ confidence level) location accuracy. (Adapted from [18]).

The actual location accuracy of the 1995 upgraded NLDN was tested in many ways. The first test was an analysis of the locations of rocket-triggered strokes, from the University of Florida's International Center for Lightning Research and Testing (ICLRT) at Camp Blanding. Figure 3.6.2 depicts a scatter-plot of the data obtained in the 1993 evaluation of 
the IMPACT algorithm [18]. Of the seven triggered strokes shown in Figure 3.6.2, three of the detected locations were within $0.6 \mathrm{~km}$ of the triggering site.

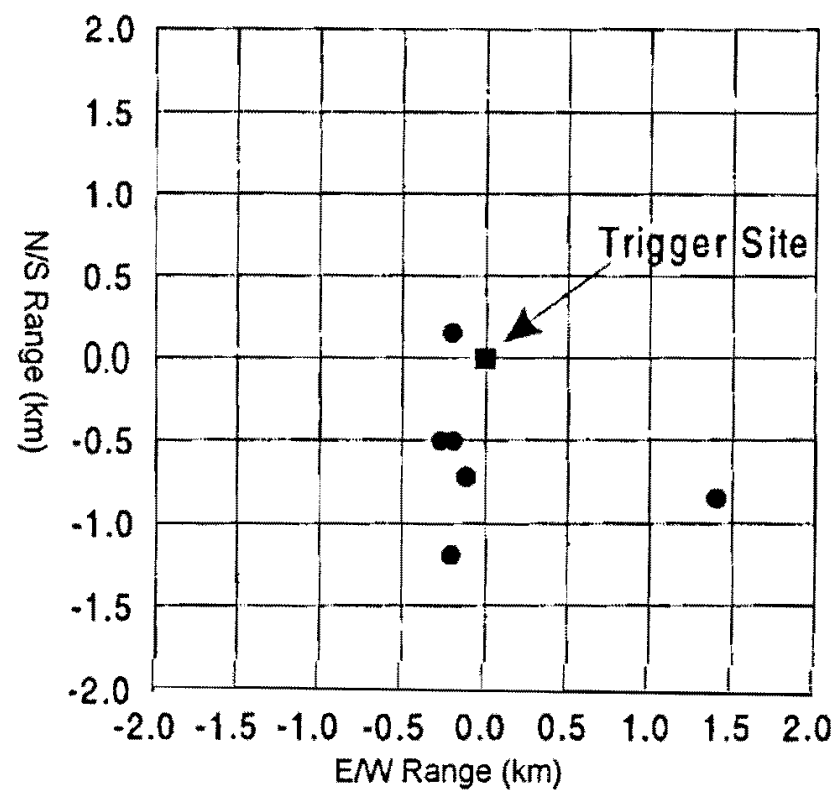

Figure 3.6.2: Locations of seven triggered strokes detected by the NLDN relative to the triggering site. (Adapted from [18].)

Comparisons were also made between the NLDN and a short baseline network of magnetic DF sensors that is operated by the NASA Kennedy Space Center and the U.S. Air Force Eastern Range at Cape Canaveral, Florida. This network has been shown to have an average location accuracy of $0.6 \mathrm{~km}$ according to [42], and therefore was a reliable reference for the NLDN. The median location difference was only $0.8 \mathrm{~km}$ between the two networks, with a standard deviation of approximately $2.3 \mathrm{~km}[18]$.

Lastly, tests were performed at the State University of New York at Albany (SUNYA) to evaluate the location accuracy of the NLDN (near Albany, New York) in 1994 and again in 1995 (before and after the NLDN upgrade). Multiple video cameras were used to locate 219 strokes independently of the NLDN in 1995 (after the upgrade), yielding a median location accuracy of $442 \mathrm{~m}$ [43]. Therefore, the results obtained from the three comparisons confirm 
the $500 \mathrm{~m}$ median location accuracy predicted for most of the U.S. in Figure 3.6.1.

As previously mentioned, in 1998 the CLDN began its first year of operation when GAI supplied, installed, and began to operate the CLDN for Environment Canada. The CLDN and the NLDN being interconnected through GAI (now Vaisala) form the NALDN, helping to improve lightning detection across North America [26]. At the time of installation, the CLDN had next generation sensors compared to those used in the NLDN. These sensors have the capability of detecting and locating intercloud and intracloud discharges; however, because these sensors are separated by 300 to $500 \mathrm{~km}$, only around 1 to $4 \%$ of cloud discharges are located by the CLDN $[25,27]$. The median stroke location accuracy for the CLDN is validated at $500 \mathrm{~m}$ using the location accuracy model [29]. However, this location accuracy should be confirmed with independent testing. The testing of median location accuracy for Southern Ontario can be easily performed using lightning data from strikes to the CN Tower, as done in this report. In fact, before the formation of the CLDN, the performance of the Ontario Lightning Location System (LLS) in 1991 was evaluated using CN Tower lightning data in [44]. The most recent projected location accuracy for the NALDN (after the 2003 NLDN upgrade) is displayed in Figure 3.6.3. It should be noted that two out of the three closest sensors to the CN Tower are NLDN IMPACT-ESP sensors.

As previously stated, the U.S. NLDN has recently undergone another upgrade which was completed in 2003. This upgrade replaced all the existing IMPACT and LPATS sensors with third generation IMPACT-ESP sensors. The median stroke location accuracy of the NLDN is still around 500 meters [24]. The 2003 upgrade has improved location accuracy in some regions, notably in the boundary areas, but the improvements are relatively small [24].

Once again, an evaluation of location accuracy was performed using rocket-triggered strokes at Camp Blanding, Florida. The following information regarding the rocket-triggered evaluation is taken from [8]. Figure 3.6.4 is a plot of the NLDN stroke location errors for 95 strokes in 31 flashes, triggered during the seasons of 2001 to 2003 . The origin corresponds to 


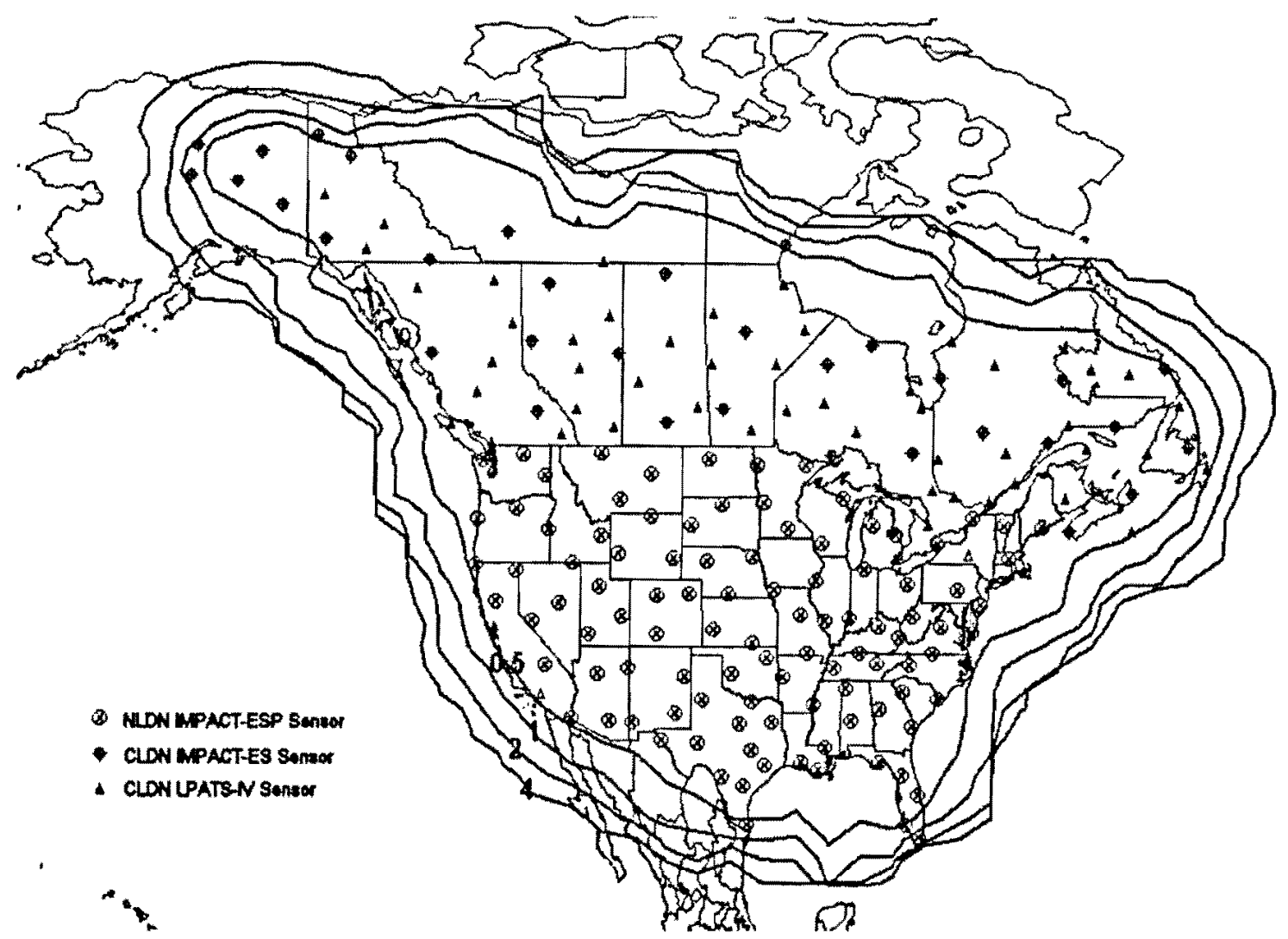

Figure 3.6.3: Projected semi-major axis of the error ellipse for the NALDN after the 2003 NLDN upgrade. Contour labels give the value in kilometers and represent the median $(50 \%$ confidence level) location accuracy. (Adapted from [5]).

the actual triggered strike location, with seven different triggering locations throughout 2001 to 2003. The median stroke location errors are $0.27 \mathrm{~km}, 0.83 \mathrm{~km}$ and $0.45 \mathrm{~km}$ for 2001,2002 and 2003, respectively. It should be noted that different sensor configurations existed from 2001 to 2003. As described in [8]: before the upgrade started in late 2001, a combination of IMPACT and LPATS-IV sensors were in the network, followed by a combination of IMPACTESP and LPATS-IV sensors in 2002, followed by only IMPACT-ESP sensors (with one left over LPATS-IV sensor) in 2003 (after the upgrade was nearly finalized). The statistics from 2001 and 2002, serve the purpose of demonstrating the effect of the final upgrade on network performance. 


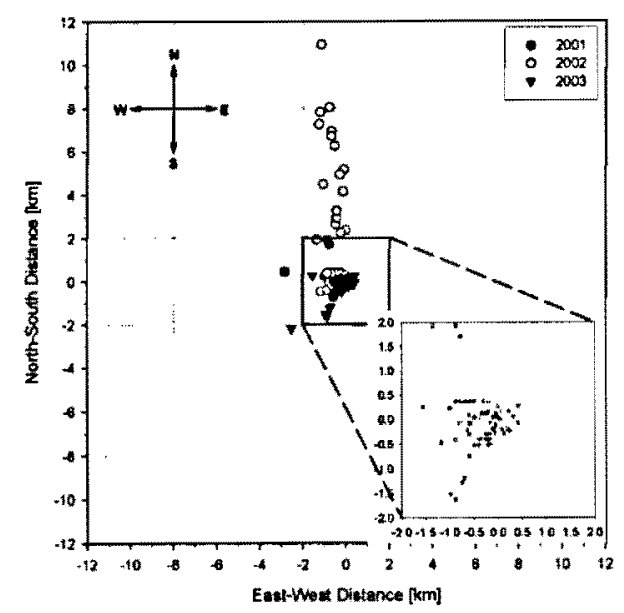

Figure 3.6.4: Plot of NLDN stroke location errors for 95 strokes in 31 flashes triggered during 2001 to 2003 at Camp Blanding. (Adapted from [8].)

The NLDN absolute location error plotted versus the peak current (measured at Camp Blanding) for 70 strokes in 22 flashes is shown in Figure 3.6.5. According to [8], the majority of large (greater than $2 \mathrm{~km}$ ) location errors in 2002 occur in the 5 to $10 \mathrm{kA}$ peak current range, with no strokes being detected for peak currents below $5 \mathrm{kA}$. For the rest of the peak currents between 10 to $35 \mathrm{kA}$, the majority of the 2002 location errors are below $1 \mathrm{~km}$. For 2003 , the location accuracy for smaller peak currents seems to have improved, but there is not enough data in that range to make any conclusions. In 2001, all of detected strokes were above $15 \mathrm{kA}$. According to [8], the sample size of 70 in Figure 3.6 .5 is smaller than the sample size of 95 in Figure 3.6.4, because the number of strokes for which peak currents were measured is smaller than the number of triggered-lightning strokes recorded.

Therefore, the peak current has a great effect on the location accuracy of the network, mostly because fewer sensors detect a low current stroke, as described later on in Section 3.6.4. Figure 3.6.6 depicts the NLDN location error versus the number of sensors used to report the stroke. When only two sensors detected a stroke, the smallest location error observed was above $2 \mathrm{~km}$. When three sensors detected a stroke, the majority of the location 


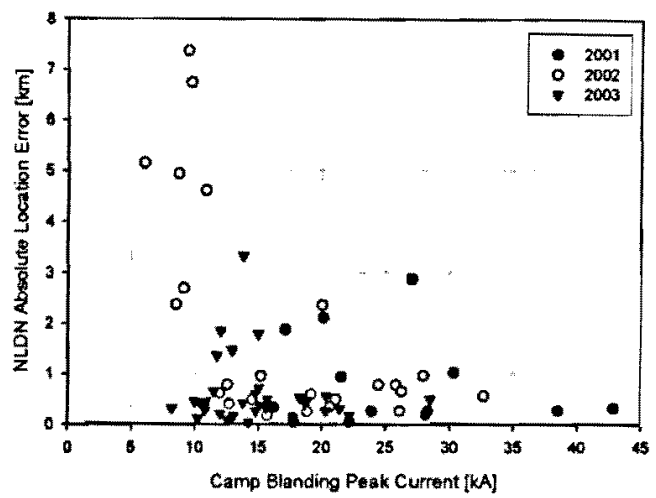

Figure 3.6.5: NLDN absolute location error versus Camp Blanding peak current, for 70 strokes with measured peak currents in 22 flashes triggered during 2001 to 2003. (Adapted from $[8]$.)

errors were between 0.1 and $3 \mathrm{~km}$. When five or more sensors reported a stroke, the location accuracy was well within $1 \mathrm{~km}$. It is clear from this data that strokes with small peak currents are detected by fewer sensors, leading to a worse NLDN location accuracy.

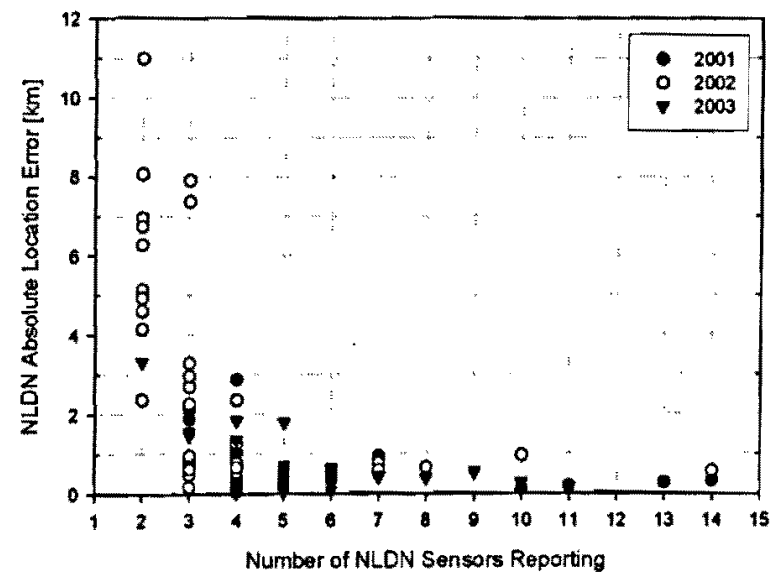

Figure 3.6.6: NLDN absolute location error plotted versus the number of reporting NLDN sensors, for 95 strokes in 31 flashes triggered during 2001 to 2003. (Adapted from [8].)

An evaluation of the error ellipse values using rocket-triggered lightning also helped confirm that the NALDN location accuracy model accurately reflects the location accuracy for lightning events. According to [8], about $66 \%$ (63 out of 95 ) and $96 \%$ (91 out of 95 ) of stroke 
locations were enclosed by the $50 \%$ and $90 \%$ error ellipses respectively, suggesting that the network near Camp Blanding is more accurate than the projections indicate.

According to [5], although these tests only validate the location accuracy in north-east Florida, this region is particulary challenging for the NLDN. The geographic characteristics of the region limit the number of sensors that are close enough to participate in the detection of strikes. If the network models correctly reflect the actual performance in this region, it is likely that projections for the rest of the network are accurate [5]. According to [8], it is important to note that triggered-lightning strokes are very similar to subsequent strokes in natural negative cloud-to-ground lightning. Therefore, the conclusions based on triggeredlightning data are applicable to subsequent strokes in negative natural downward lightning, but not necessarily to natural negative first strokes or positive strokes [8].

\subsubsection{Flash Detection Efficiency}

The NLDN detection efficiency (DE) for flashes with first stroke peak currents greater than $5 \mathrm{kA}$ was approximately $70 \%$ before 1992 , and $65 \%$ to $80 \%$ from 1992 to 1995 [1]. According to [18], the 1995 NLDN upgrade was designed to provide typical flash DEs in the range of $80 \%$ to $90 \%$, for first strokes with peak currents of $5 \mathrm{kA}$ and larger. It should be noted that a $5 \mathrm{kA}$ threshold is thought to include $95 \%$ of all negative first strokes [35]. The flash DE model projection for negative flashes is shown in Figure 3.6.7. The model estimates did not include flashes with peak currents below $5 \mathrm{kA}$, because of the large uncertainties in the peak current distribution at these low values [18]. According to [1], strokes having a peak current below $5 \mathrm{kA}$ were ususally not detected because the signals fall below the trigger threshold of multiple sensors. Conversely, strokes with a peak of $100 \mathrm{kA}$ are typically detected by 20 or more sensors, while a common first return stroke peak of $25 \mathrm{kA}$ is detected by six to eight sensors [1]. A significant fraction of subsequent strokes in multiple-stroke 
flashes was not detected because of this $5 \mathrm{kA}$ limit [1]. It is interesting to note once again that the DE in Southern Ontario is $80 \%$ according to the model. As shown in Figure 3.6.7, the DE falls off rapidly as the perimeter of the network is approached near sea coasts and borders.

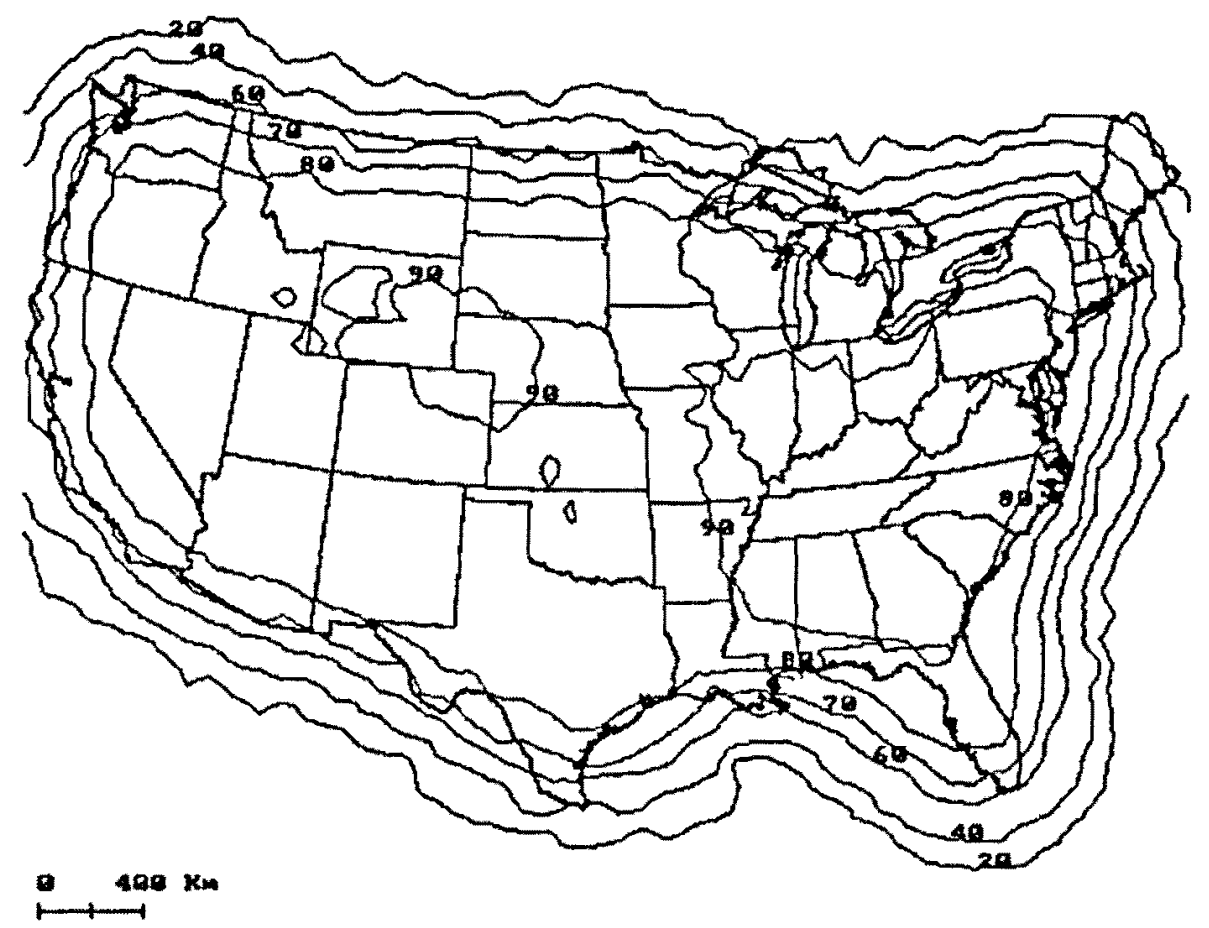

Figure 3.6.7: Projected flash DE for the NLDN after the 1995 upgrade for negative flashes with peak currents greater than $5 \mathrm{kA}$. Contour labels give the $\mathrm{DE}$ in percent. (Adapted from $[18])$

To verify the DE in Figure 3.6.7, tests were performed at the State University of New York at Albany (SUNYA) near Albany, New York, using video cameras [45]. After the 1995 upgrade was finalized, this study showed that the flash DE was $84 \%$ for flashes with peak currents over $5 \mathrm{kA}$ [45]. Once again, it should be noted that a $5 \mathrm{kA}$ threshold is thought to include $95 \%$ of all negative first strokes [35]. The study did find that the stroke DE greatly depended upon peak currents, as discussed in next section. 
As previously mentioned, after the 1995 upgrade of the NLDN, the location accuracy and DE still fell off near the edges of the network. The addition of the CLDN in 1998 eliminated the limitations on the northern border of the NLDN, but the coastal and southern borders continued to have location accuracy and DE limitations. An analysis of the CLDN by GAI estimated that the flash DE is 85 to $90 \%$ out to $200 \mathrm{~km}$ from the network periphery, decreasing to $80 \%$ at the periphery, and 10 to $30 \%$ at a distance of $300 \mathrm{~km}$ beyond the periphery $[25,29]$. According to [29], the flash DE has been verified to range from 80 to $90 \%$ across most of the ten provinces for strokes with peak currents above $5 \mathrm{kA}$. As previously mentioned, the CLDN can also detect intercloud and intracloud discharges; however, because these sensors are separated by 300 to $500 \mathrm{~km}$, only around 1 to $4 \%$ of the cloud discharges are located by the CLDN $[25,27]$. The most recent projected flash DE for the NALDN (after the 2003 NLDN upgrade) is displayed in Figure 3.6.8.

Independent testing of the flash DE in Southern Ontario can be easily performed using CN Tower lightning data. Furthermore, individual stroke detection efficiency can also be determined for a variety of peak currents, using the accumulated current data from the $\mathrm{CN}$ Tower. Two out of the three closest sensors to the CN Tower are NLDN IMPACT-ESP sensors, which have recently been upgraded and require performance validation.

One of the goals of the 2003 upgrade of the NLDN was to improve location accuracy and DE on the boundary of the network. The projected flash DE for the NALDN after the 2003 NLDN upgrade is displayed in Figure 3.6.8. According to [5], there is no longer a $5 \mathrm{kA}$ lower limit on the estimated flash DE; however, rocket-triggered evaluations for stroke DE prove otherwise, as discussed in the next section. The location accuracy results from the evaluation performed using rocket-triggered strokes (at Camp Blanding, Florida) were discussed previously in Section 3.6.1, where a description of the sensors used from 2001 to 2003 was given. According to [8], since all triggered flashes contain only strokes similar to subsequent strokes in natural negative downward lightning (i.e. contain no first 


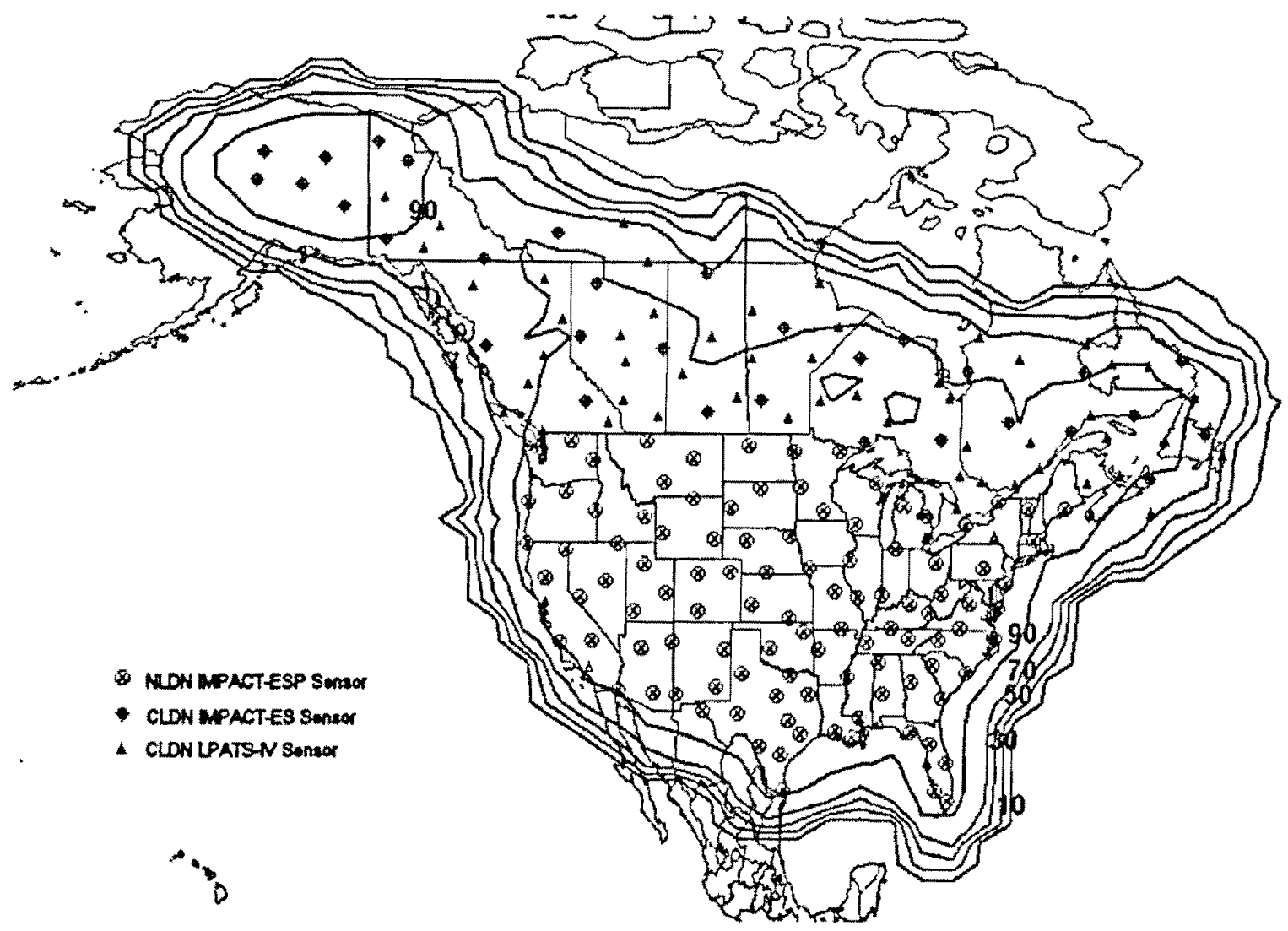

Figure 3.6.8: Projected flash DE for the NALDN after the 2003 NLDN upgrade. Contour labels give the DE in percent.(Adapted from [5].)

strokes), the flash DE reported may be an underestimate of the true value for natural negative lightning flashes in Florida. Also, the reported flash DE efficiency may be applicable to upward lightning initiated from tall objects, since upward flashes are thought to be similar to triggered flashes [8]. The flash DE was $82 \%$ (9 out of 11) in 2001, 86\% (12 out of 14) in 2002 , and $83 \%$ (10 out of 12) in 2003 [8]. These values are in fairly good agreement with the flash DE model of Figure 3.6.8, particulary since the reported DE may be an underestimate, as noted above.

Another evaluation of the 2003 upgrade of the NLDN was performed by the University of Arizona in [6]. During the summer of 2003, a digital video recording system with GPS time 
was used to record 671 cloud-to-ground flashes in 18 thunderstorms near Tucson, Arizona [6]. According to [6], the average flash DE of the NLDN near Tucson was approximately $95 \%$ During 2001, the same video system was used to record 109 flashes in 5 thunderstorms, resulting in a flash DE of $71 \%$ [ 7$]$. The better flash DE in 2003 is most likely because of the network upgrade.

\subsubsection{Stroke Detection Efficiency}

After the 1995 upgrade of the NLDN, the stroke DE was estimated as being 40 to $50 \%$ [24]. According to [8], initially the estimated stroke DE was thought to be roughly $50 \%$ for the network, based on a comparison of the average NLDN stroke multiplicity of about 2 (observed for two years after the 1995 upgrade) and the average stroke multiplicity of 3 to 4 reported in [46]. Subsequent strokes are thought to have peak currents that are about half as large as those in first strokes, making them harder to detect. [18]. To verify the stroke DE, tests were performed at the State University of New York at Albany (SUNYA) (near Albany; New York) using rideo cameras [45]. After the 1995 upgrade was finalized, this study showed that the stroke DE was $66 \%$ for strokes with peak currents over $5 \mathrm{kA}$, and $47 \%$ for all strokes including those with currents below $5 \mathrm{kA}[35,45]$. The study found that the stroke DE greatly depended upon peak currents. As described in [35] and [45], the XLDN detected strokes that had estimated peak currents orer $16 \mathrm{kA}$ with a DE of about $97 \%$ ( 38 out of 39 ); the DE was about $15 \%$ for strokes in the 6 to $10 \mathrm{kA}$ range and zero for strokes below $6 \mathrm{kA}$.

Aftcr the 2003 upgrade of the NLDN, the stroke DE of the network increased to approximately 60 to $50 \%$ [24]. Evaluations indicate that a greater amount of smaller subsequent strokes are being detected. This increase in stroke DE also led to the increase in the flash DE noted in the previous section. According to [8], the stroke DE reported by the rocket- 
triggered evaluation at Camp Blanding in Florida is probably representative of the true subsequent stroke DE for natural lightning in Florida, but not the overall stroke DE of the whole network. Also according to [8], the flash and stroke DEs reported may be applicable to upward lightning initiated from tall objects, since upward flashes are thought to be similar to triggered flashes. The observed stroke DE was $52 \%$ (17 out of 33 ) in $2001,57 \%$ (44 out of 77 ) in 2002 and $69 \%$ (34 out of 49 ) in 2003, with the sensor set-up as described initially in Section 3.6.1 for each year. The $69 \%$ value which was obtained after the 2003 upgrade, is quite an improvement over the stroke DE before the upgrade.

The following information is summarized from [8]. Figure 3.6.9 displays the NLDN stroke $\mathrm{DE}$ as a function of peak current measured at Camp Blanding. According to [8], strokes for which no current was measured (due to instrumentation failure) at Camp Blanding are not included, reducing the total number of strokes in Figure 3.6.9 to 122 (although some of the strokes without measured currents may have been detected by the NLDN). As shown in Figure 3.6.9, for 2001 no strokes with peak currents below $5 \mathrm{kA}$ were triggered, but seven strokes were triggered having peak currents from 5 to $15 \mathrm{kA}$, none of which were detected by the NLDN. Above $15 \mathrm{kA}$, stroke DE increases reaching $100 \%$ between 25 and $30 \mathrm{kA}$. Because the sample size is small, particularly for the larger currents, these results may not accurately depict the stroke DE. For 2002, three strokes with peak currents below $5 \mathrm{kA}$ were triggered, but none were detected. Above $5 \mathrm{kA}$, stroke $\mathrm{DE}$ remained roughly constant at $40 \%$ to $50 \%$, until it increased to $100 \%$ at 25 to $30 \mathrm{kA}$. Once again, due to the small sample size for larger strokes, the results may not be fully representative of the true stroke DE. In 2003 , three strokes with peak currents below $5 \mathrm{kA}$ were triggered, but once again as in 2002 , none of them were detected. Peak currents from 5 to $10 \mathrm{kA}$ had a stroke DE of $20 \%$, but the $\mathrm{DE}$ increased to $100 \%$ above $10 \mathrm{kA}$, except for the 25 to $30 \mathrm{kA}$ range where one out of two strokes was detected. Since the sample size of two is very small, this observation is definitely not indicative of the true stroke DE for larger strokes. Clearly the stroke DE 
after the 2003 upgrade has greatly improved according to this evaluation, however, strokes under $5 \mathrm{kA}$ were still not detected. Low current strokes continue to be problematic for the network, even though they are not supposed to be according to [5]. However, the sample size was very small for all strokes and more evaluations are needed.
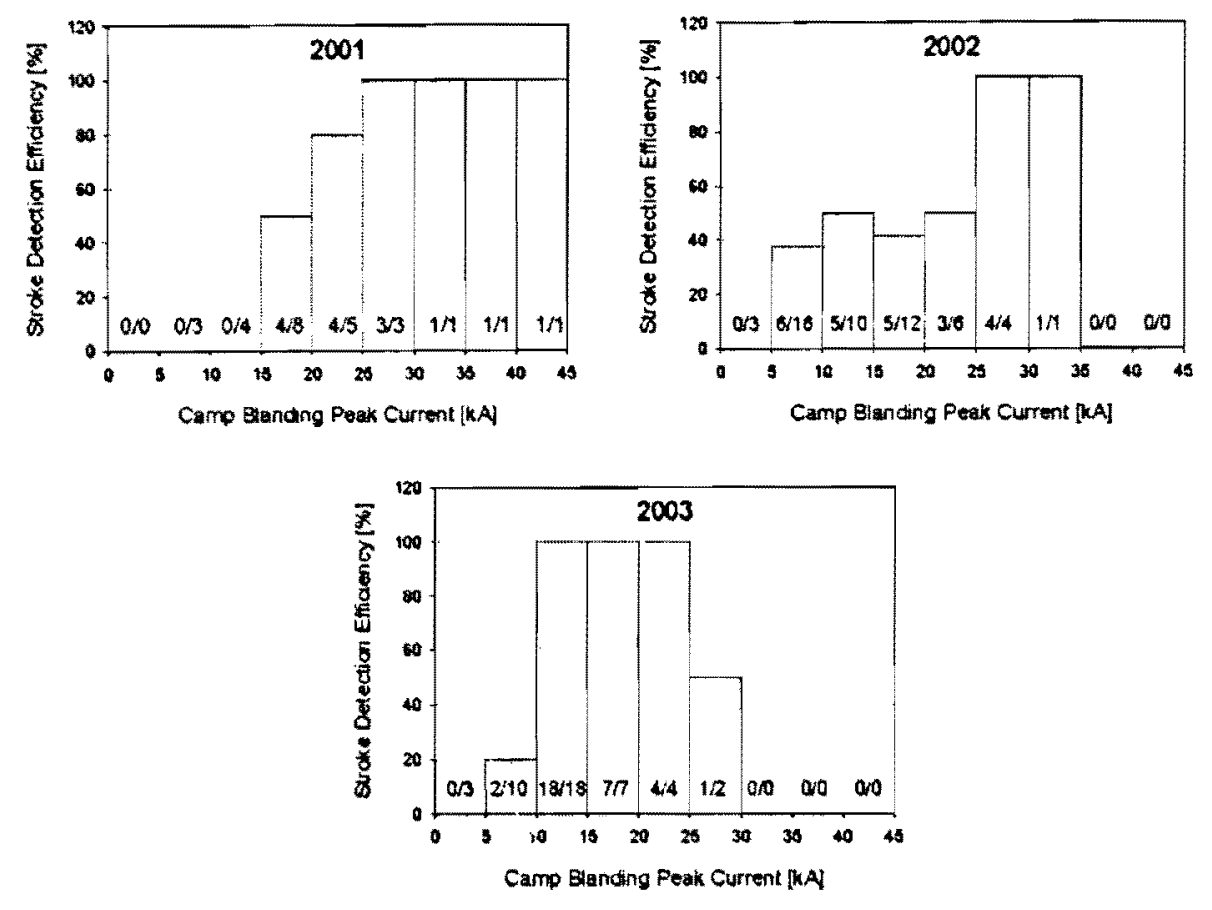

Figure 3.6.9: NLDN stroke detection efficiency plotted as a function of peak current measured at Camp Blanding. The total number of strokes whose currents were measured at Camp Blanding is 122, of which 70 were detected by the NLDN. (Adapted from [8].)

Results from the evaluation performed during the summer of 2003 by the University of Arizona near Tucson, Arizona (using a digital video recording system with GPS time to record 671 cloud-to-ground strikes in 18 thunderstorms), are described below [6]. According to [6], the 671 flashes contained at least 2290 separate return strokes, and the average stroke DE of the NLDN near Tucson was approximately 78\%. During 2001, the same video system was used to record 109 Hashes containing 401 strokes in 5 thunderstorms, resulting in a stroke DE of $41 \%[7]$. 


\subsubsection{Peak Current}

The following information regarding peak current estimation after the 1995 NLDN upgrade is summarized from [1] and [18]. After the 1995 upgrade, the NLDN had a median error of 20 to $30 \%$ in current estimation, with the larger errors being associated with smaller currents. This estimate is based on a comparison with the measured currents of negative rocket-triggered lightning strokes. As aforementioned, rocket-triggered lightning strokes are more representative of natural negative subsequent strokes. The NLDN current estimates should always be viewed with caution for strokes other than negative subsequent strokes, because no NLDN current estimates exist for: first strokes in natural lightning, natural positive strokes, or strokes exceeding $60 \mathrm{kA}$.

The CN Tower provides an excellent opportunity to evaluate peak current estimation in Southern Ontario. Evaluations of the peak current in the 1989 Ontario LLS were performed in [13] and [47]; however, a more recent evaluation is obviously needed since the formation of the CLDN in 1998.

The follwing results from the rocket-triggered evaluation at Camp Blanding, performed from 2001 to 2003, are summarized from [8]. Figure 3.6.10 shows the NLDN peak current estimation error (defined as NLDN estimated peak current minus Camp Blanding measured Peak Current) plotted versus Camp Blanding peak current, for 70 strokes in 22 flashes triggered during 2001 to 2003 . Clearly there is some correlation between the NLDN peak current estimation error and the peak current measured. It is important to note that $\Delta I$ is not an absolute value and that the NLDN seems to underestimate the actual peak current for most strokes. Also, most of the NLDN peak current estimation errors are below $5 \mathrm{kA}$. According to [8], for the unsigned (absolute) percentage errors, the median values were $25 \%$, $17 \%$ and $23 \%$, for 2001,2002 and 2003 , respectively. Therefore, the median peak current estimation error is roughly $20 \%$. 

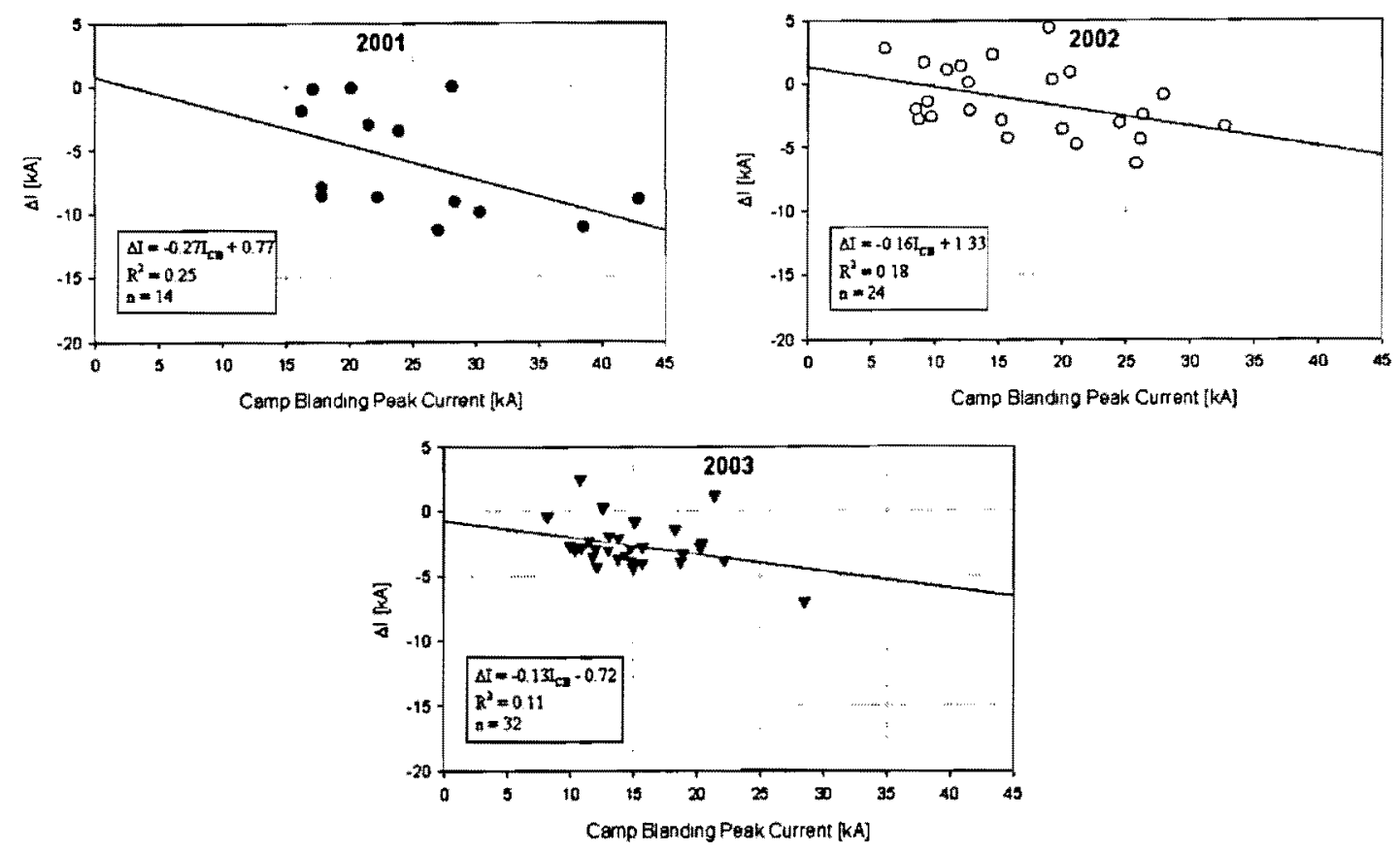

Figure 3.6.10: NLDN peak current estimation error (defined as $\triangle I=I_{N L D N}-I_{C B}$ [NLDN estimated peak current minus Camp Blanding measured Peak Current]) plotted versus Camp Blanding peak current, for 70 strokes in 22 flashes triggered during 2001 to 2003. (Adapted from [8].)

The number of NLDN reporting sensors is plotted against Camp Blanding peak current in Figure 3.6.11. As expected, there is a positive correlation between measured peak current and the number of reporting sensors, since larger peak currents should correspond to larger signal strengths at NLDN sensor locations. The range of the number of NLDN reporting sensors is quite large for most peak currents; for example, for strokes ranging from 20 to 30 $\mathrm{kA}$, the number of reporting sensors ranges from 4 to 11. According to Figure 3.6.11, for peak currents under $10 \mathrm{kA}$ there are usually three or fewer reporting sensors. This explains why the stroke location accuracy and DE fall off rapidly in this peak current range, as mentioned in previous sections. Also as mentioned in the previous section, strokes with peak currents of $5 \mathrm{kA}$ and less were not detected by any NLDN sensors at all. 


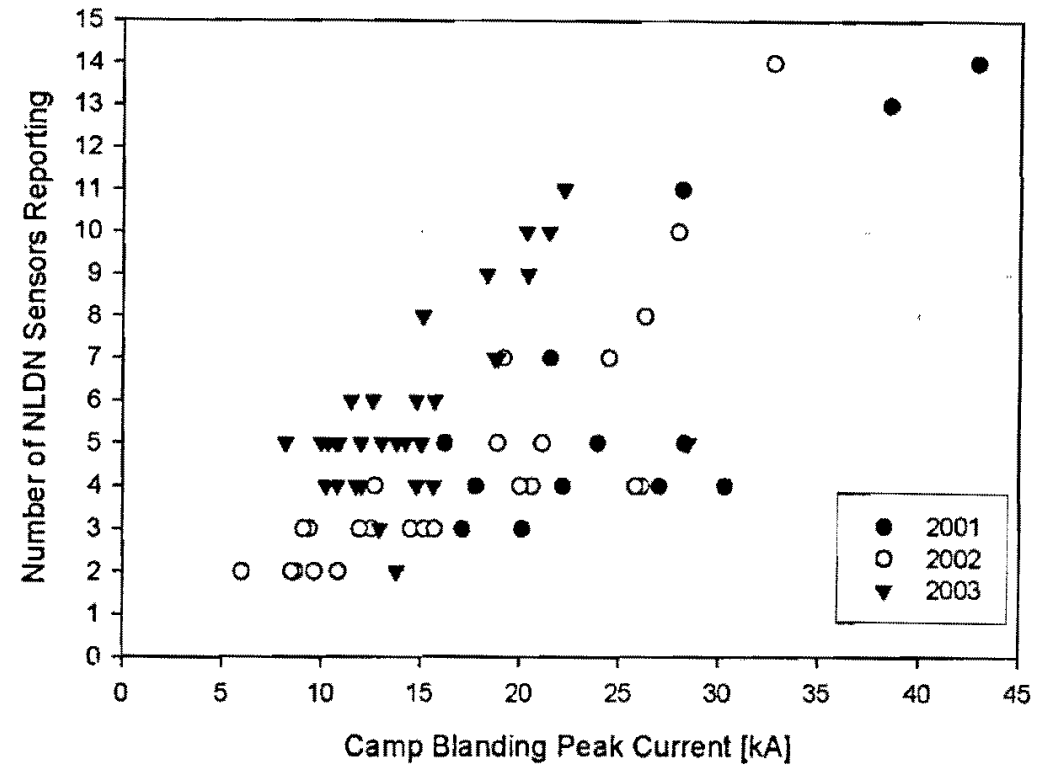

Figure 3.6.11: Number of NLDN reporting sensors plotted versus Camp Blanding peak current, for 70 strokes with measured peak currents in 22 flashes triggered during 2001 to 2003. (Adapted from [8].)

\subsubsection{Misclassified Events}

The following information regarding the 1995 upgrade of the NLDN is summarized from [1] and [18]. After the 1995 upgrade, the NLDN recorded many small positive lightning events with peak currents below $10 \mathrm{kA}$, which were not detected previously. In 1995 , the total number of positive discharges detected was approximately two times greater than in 1994. Because of the increased sensitivity of the upgraded system, these small positive events were actually cloud-to-cloud discharges that were not previously detected. It was recommended in [18] that small positive discharges with peak currents less than $10 \mathrm{kA}$ be regarded as cloud discharges, unless verified as being cloud-to-ground events. Many of the newly detected positive discharges also had peak currents between 5 to $15 \mathrm{kA}$, making it likely that some of these of events were also misappropriated cloud-to-cloud events.

The CLDN has the same problem, although less severe. In [25], the $10 \mathrm{kA}$ limit was 
applied to the whole NALDN, with all events below $10 \mathrm{kA}$ regarded as cloud discharges. According to [25], although the CLDN sensors are less susceptible to the misclassification of cloud flashes than the NLDN sensors, the NLDN sensors contribute data up to $500 \mathrm{~km}$ into Canada, and well into Southern Ontario. Therefore, the $10 \mathrm{kA}$ limit is being applied to the whole NALDN in statistical studies.

The following information regarding the 2003 upgrade of the NLDN is summarized from [5]. According to [5], refinements in the NLDN's location algorithm and waveform classifcation were made to reduce the cloud-to-cloud events from being misclassified as cloud-toground events. These refinements reduced the number of misinterpreted events but did not completely eliminate the problem. Because of the even greater enhanced sensitivity of the IMPACT-ESP sensors, the percentage of misappropriated cloud-to-cloud events is actually larger than before the 2003 upgrade. Although the percentage of misclassified positive discharges with peak currents greater than $10 \mathrm{kA}$ was reduced, the small positive discharges with peak currents below $10 \mathrm{kA}$ are still being misclassified. According to [5], the University of Arizona study has confirmed that approximately $90 \%$ of the small positive events with currents under $10 \mathrm{kA}$ are actually cloud discharges, and that most larger positive events with currents above $20 \mathrm{kA}$ are cloud-to-ground strokes. These studies also indicate that nearly all of the negative polarity small peak current events in Texas, are in fact cloud-to-ground events. More sophisticated classification methods for cloud-to-cloud and cloud-to-ground events are currently being examined. In the meantime, a temporary solution is underway to create an "ambiguous" category, to assign the events that cannot be clearly classified.

\subsubsection{Summary}

Table 3.6.1 provides a general overview of the characteristics and overall projected performance of the NALDN. 


\begin{tabular}{|l|l|l|l|}
\hline Basic Parameters & $\begin{array}{l}\text { NLDN: After 1995 Up- } \\
\text { grade }\end{array}$ & $\begin{array}{l}\text { NLDN: After 2003 Up- } \\
\text { grade }\end{array}$ & CLDN \\
\hline Number of sensors & 106 & 113 & 81 \\
\hline $\begin{array}{l}\text { Types of lightning } \\
\text { sensors }\end{array}$ & $\begin{array}{l}\text { First generation IMPACT } \\
\text { sensors and older LPATS-III } \\
\text { sensors }\end{array}$ & $\begin{array}{l}\text { Third generation IMPACT- } \\
\text { ESP sensors }\end{array}$ & $\begin{array}{l}\text { Second } \\
\text { IMPACT-ES } \\
\text { LPATS-IV sensors }\end{array}$ \\
\hline $\begin{array}{l}\text { Sensing technol- } \\
\text { ogy }\end{array}$ & $\begin{array}{l}\text { Combined magnetic DF / } \\
\text { TOA sensors and TOA only } \\
\text { sensors }\end{array}$ & $\begin{array}{l}\text { Combined magnetic DF } \\
\text { TOA sensors } \\
\text { and } \\
\text { ficiency }\end{array}$ & $\begin{array}{l}\text { Combined magnetic DF / } \\
\text { TOA sensors and TOA only } \\
\text { scnsors }\end{array}$ \\
\hline $\begin{array}{l}\text { Stroke detection } \\
\text { efficiency }\end{array}$ & $80-90 \%$ & $80-90 \%$ \\
\hline $\begin{array}{l}\text { Stroke location ac- } \\
\text { curacy }\end{array}$ & $500 \mathrm{~m}$ median error & $500 \mathrm{~m}$ median error & $40-50 \%$ \\
\hline $\begin{array}{l}\text { Network uptime } \\
\text { Minimum peak } \\
\text { current detected } \\
\text { (1st percentile) }\end{array}$ & As low as 5-8 kA & As low as 3-4 kA & $500 \mathrm{~m} \mathrm{median} \mathrm{error}$ \\
\hline
\end{tabular}

Table 3.6.1: Summary of the NALDN projected performance. (Adapted from [24].) 


\subsection{Applications}

The NALDN is the number one source for lightning occurrence information in North America. The main applications and uses of NALDN data are listed in Chapter 1, the Introduction, in order to immediately illustrate the importance of the NALDN. According to Vaisala, the company which runs the NALDN, the data acquired by the network is regularly used by [30]:

- The National Weather Service

- Environment Canada (which owns NALDN lightning data for Canada)

- The Federal Aviation Administration

- The Weather Channel

- The Professional Golfers' Association (PGA) Tour

- Major power companies

- International and regional airports

- Thousands of businesses

The NALDN data is used for monitoring current conditions in real-time and for studying past events. Since 1989, the NLDN has reported more than 20 million cloud-to-ground lightning flashes that occur every year in the U.S. [4]. In 1998, the CLDN began reporting cloud-to-ground lightning flashes, creating an archive of lightning data along with the NLDN which can be used for statistical and forensic analysis [29].

Meteorologists, electric power utilities, arson investigators, insurance companies, and land management agencies use historic NALDN data to correlate and document suspected lightning damage with recorded lightning activity [29]. Seasonal or multi-year studies of lightning trends in a specific area are also important for lightning risk assessment, site selection, and designing optimal lightning protection schemes [29]. 
The CLDN has detected over 5 million flashes annually since 1999, and approximately 10 million flashes in 2002 [33]. During days of heavy or widespread thunderstorms, the CLDN will detect 15,000 to 22,000 flashes per hour; the 15,000 flashes per hour rate occurred 38 times during 2002 [33]. In a typical year it is estimated that an average of seven people will die from being struck by lightning in Canada, and 60 to 70 people will be seriously injured with long term effects [2]. It is also estimated that 40,000 insurance claims will be filed in Canada this year due to lightning, with payouts in excess of 40 million dollars for property damage [33]. Timber losses can run into the billions of dollars per year, as lightning is responsible for approximately $42 \%$ of all forest fires, having caused loses estimated at 14 billion dollars annually between 1979 and 1993 [3].

As previously mentioned, the NALDN is extremely important for human safety, protection of property, and legal issues such as insurance claims. As shown in Section 3.1, this field has quickly developed from an educational pursuit to a very profitable commercial venture because of the vast need for accurate lightning location in many applications.

\subsection{Summary}

A comprehensive overview of the NALDN was provided in this chapter. The history and development of the NALDN was presented, detailing how the Vaisala Group from Finland came to own the U.S. NLDN, and also detailing how the CLDN was established in 1998, with Environment Canada owning the network and Vaisala operating it. The NLDN and CLDN are completely interconnected to form the NALDN, with all data being processed by Vaisala in Tuscon, Arizona. The sensor locations of the NALDN were also shown, with the CLDN consisting of IMPACT-ES and LPATS-IV sensors and the NLDN consisting of third generation IMIPACT-ESP sensors. The NALDN estimates the time of a stroke, location of a stroke, polarity of a stroke, peak current of a stroke and the confidence ellipse of the stroke. 
The lightning data is available in real-time within 30 to $40 \mathrm{~s}$ of the event occurrence.

The IMPACT method is used to detect and locate lightning events in the NALDN. This method can use information from any combination of magnetic DF sensors, TOA sensors and IMPACT sensors. In the NALDN, strokes are added to a flash for a period of $1 \mathrm{~s}$ after the first stroke, if the additional strokes are within $10 \mathrm{~km}$ of the first stroke and the time interval from the previous stroke is less than $500 \mathrm{~ms}$. If a stroke happens to qualify as a part of more than just one flash, it is grouped into the flash with the closest first stroke. Furthermore, a stroke is included in a flash if it is located within 10 to $50 \mathrm{~km}$ of the first stroke and the $50 \%$ confidence ellipses of the strokes overlap. The polarity of a stroke is determined using the measured electric field peak. The time of a stroke is derived in the IMPACT optimization algorithm, and since the stroke position is uncertain to within a few hundred meters (500 m median accuracy), the onset time should be accurate to approximately $5 \mu \mathrm{s}$. The peak current for each stroke is also estimated, using the relationship between peak field and peak current, which assumes that the simple transmission line model (TLM) is valid for the peak field. The NALDN location accuracy model produces a confidence ellipse for each stroke, which defines a region centered around the computed stroke location where there is a certain probability that the stroke occurred. The DE model computes estimates of the DE on a 50-by-50 grid over the region of network coverage. The model generates specific values of peak current at each grid point, and computes the signal strength that should arrive at each sensor.

A comparison of past and present performance for the NALDN was also provided in the latter part of this chapter. The projected flash DE for the NLDN is currently $90 \%$ or better, while for the CLDN it is 80 to $90 \%$ depending on the region. The projected stroke DE for the NLDN is currently 60 to $80 \%$, whereas for the CLDN it is 40 to $50 \%$. Both the NLDN and CLDN have a $500 \mathrm{~m}$ median location accuracy. Lastly, the applications of the NALDN were outlined, showing how data from the network is regularly used by The National Weather 
Service, Environment Canada, The Federal Aviation Administration, The Weather Channel, The Professional Golfers' Association (PGA) Tour, major power companies, airports and thousands of other businesses. 


\section{Chapter 4}

\section{North American Lightning Detection Network Performance Analysis using CN Tower lightning data}

The results of the North American Lightning Detection Network (NALDN) evaluation in the Toronto area are presented in this chapter. Initially the $\mathrm{CN}$ Tower lightning project is discussed, including the history and development of the project and the equipment used to acquire CN Tower lightning data. A summary of the CN Tower lightning project is then provided, where the manner in which the CN Tower data is used to evaluate NALDN data is

explained. Afterwards, the performance characteristics of the NALDN are evaluated using the CN Tower lightning data acquired over the Toronto lightning season in 2005. A detailed analysis of the NALDN flash detection efficiency, stroke detection efficiency, absolute location error, peak current estimation and location accuracy model (50\%, 90\% and $99 \%$ error ellipses) is given, followed by an in-depth discussion of the results. 


\subsection{The CN Tower Lightning Project}

\subsubsection{Overview}

Standing at a height of $553 \mathrm{~m}$, the CN Tower is the tallest freestanding structure in the world. Lightning strikes to the CN Tower have been observed since 1978, two years after its construction, and although the lightning flash density is less than 2 flashes per square kilometer in Toronto, the CN Tower receives several tens of strikes each year [9]. The CN Tower is one of the best sites in the world to observe the lightning phenomenon at tall structures and to obtain definitive lightning data such as currents, magnetic and electric fields, and visual parameters.

By 1991 the CN Tower was equipped with a current derivative measurement system and a laboratory was established $2 \mathrm{~km}$ north of the $\mathrm{CN}$ Tower to measure the vertical component of the electric $\left(\mathrm{E}_{z}\right)$ field and the azimuthal $\left(\mathrm{H}_{\phi}\right)$ and radial $\left(\mathrm{H}_{r}\right)$ components of the magnetic fields generated by lightning strikes. A more detailed description of the current derivative measurement system and the field measurement system is provided in Section 4.1.2 and Section 4.1.3, respectively. Three Tektronix RTD710A (10 bit, $10 \mathrm{ns)} \mathrm{computer} \mathrm{controlled}$ double-channel digitizers with segmented memories were used to simultaneously record the return stroke current derivative at the $\mathrm{CN}$ Tower and the corresponding electric and magnetic fields at the laboratory $2 \mathrm{~km}$ north of the Tower [48]. A VHS recording system was also installed at this laboratory to record the visual parameters of the lightning strikes.

By 1996, a Phantom V2.0 1000 frame/sec High-Speed Camera was acquired and installed at the laboratory. In 1997, a new current derivative measuring system that is noise protected was installed at the $\mathrm{CN}$ Tower. By 2002, the three outdated Tektronix digitizers were replaced with two LeCroy LT342L ( 8 bit, 2 ns) digitizers. A Truetime XL-DC GPS timing system accurate to $1 \mathrm{~ms}$ was also installed on the VHS recording system, allowing for the precise timing of lightning flashes. In 2004, a Truetime 560-5908 PCI-SG2 GPS timing 
system accurate to $1 \mu \mathrm{s}$ was installed for the $\mathrm{CN}$ Tower current derivative measurement system, and also for the electric and magnetic field measurement system. This extremely accurate timing system now allows for the comparison of CN Tower lightning strokes to the strokes detected by the NALDN. Recently in the summer of 2005, an APC Battery Backup XS 1000 uninterruptible power supply was also installed at the CN Tower, in order to ensure that operation continues in the case of power surges or short blackouts. The VHS recording system is also in the process of being completely replaced with a 160GB hard drive DVD recording system, based on the JVC DR-MH30 DVD video recorder.

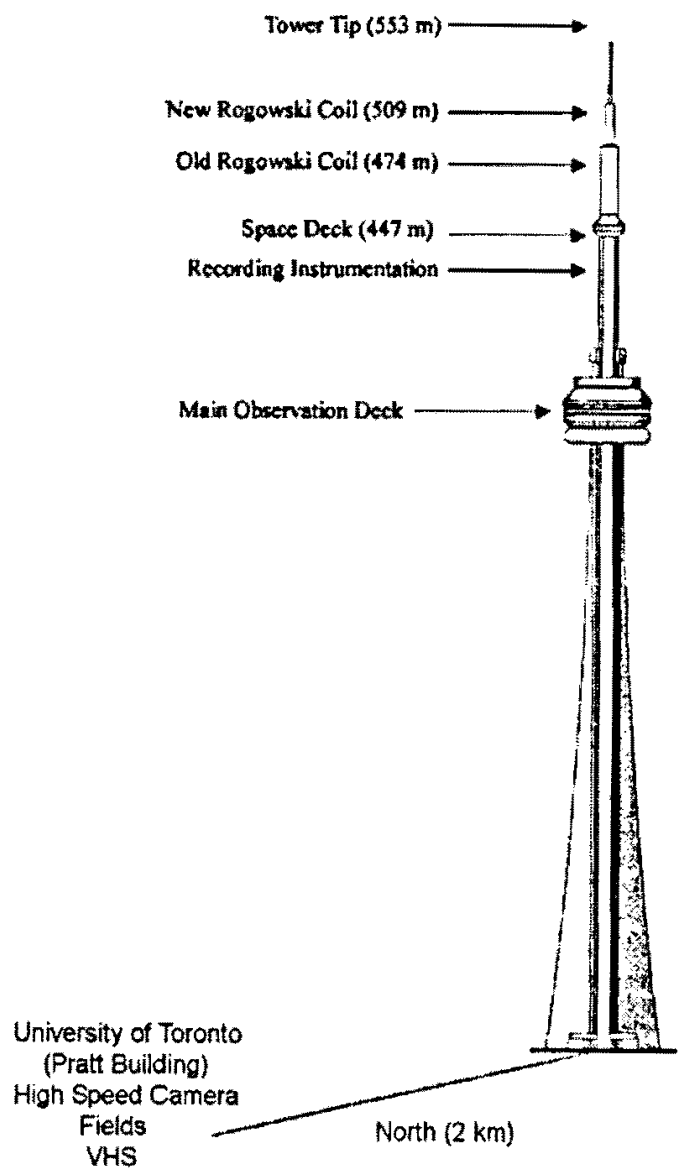

Figure 4.1.1: The CN Tower and measurement system locations. (Adapted from [48].) 


\subsubsection{Current Derivative Measurement System}

In 1990 , a $3 \mathrm{~m}, 40 \mathrm{MHz}$ Rogowski current sensing coil was installed at the CN Tower at $474 \mathrm{~m}$ above ground level as shown in Figure 4.1.1 [48]. As shown in Figure 4.1.2, the CN Tower's steel structure contains five identical plates that are joined to form a cylinder with a pentagon cross-section [48]. Each plate contains an elliptical opening and the Rogowski coil passes through two adjacent openings, encircling one fifth of the CN Tower. Because the coil encircles one fifth of the Tower, it is assumed that the recorded signal corresponds to $20 \%$ of the total current [48]. The coil is connected via a triaxial cable to a recording system at $403 \mathrm{~m}$ above ground level, as shown in Figure 4.1.1 [48]. The recording system currently consists of a LeCroy LT342L ( 8 bit, 2 ns) digitizer and a computer. The system also contains a recently installed GPS timing system, which is accurate to $1 \mu \mathrm{s}$. The Rogowski coil has a risetime of $8.7 \mathrm{~ns}$, a sensitivity of $0.32 \mathrm{~V} /(\mathrm{A} / \mathrm{ns})$, and an accuracy of $\pm 6 \%$, while the overall risetime of the measurement system is estimated to be approximately $20 \mathrm{~ns}$ [48].
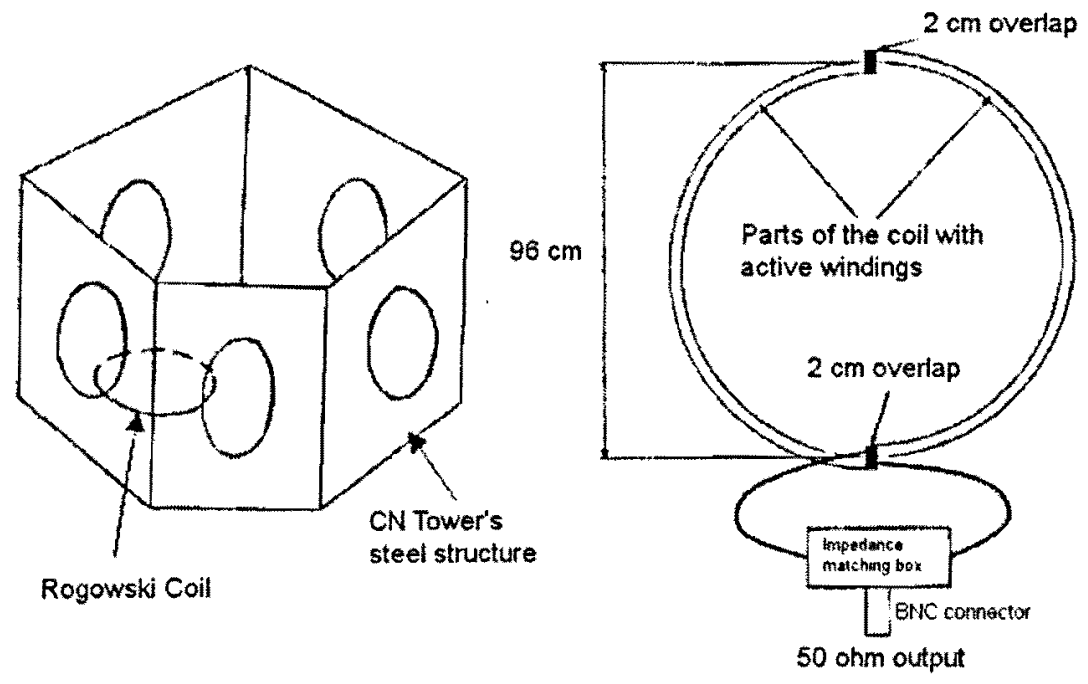

Figure 4.1.2: The old Rogowski coil and its location. (Adapted from [48].)

In 1997 , a noise protected current derivative measurement system was installed at the 
CN Tower at $509 \mathrm{~m}$ above ground level. This system features a $6 \mathrm{~m}, 20 \mathrm{MHz}$ Rogowski coil which surrounds the entire steel structure of the CN Tower and is connected to the recording system via a optical fibre link [48]. This new coil captures the current derivative with a vastly improved signal-to-noise ratio over the old coil, because the whole current derivative signal is captured and an optical fibre link is used instead of a triaxial cable [48].

The old coil is currently connected to the second channel of the LeCroy LT342L (8 bit, $2 \mathrm{~ns}$ ) digitizer. The new coil is connected to the first channel of the digitizer and is used to trigger the digitizer whenever a lightning strike occurs, in order to capture the waveform. Once the digitizer is triggered, it subsequently outputs a trigger to the Truetime $560-5908$ PCI-SG2 GPS timing system, which will immediately record the GPS time with an accuracy of $1 \mu \mathrm{s}$. As previously mentioned, the overall risetime of the system is approximately $20 \mathrm{~ns}$, so the accuracy of the GPS time is unaffected. This recording system currently uses an APC Battery Backup XS 1000 uninterruptible power supply, in order to ensure that operation continues in the case of short blackouts or power surges.

\subsubsection{Field Measurement System}

Since 1991, the vertical component of the electric field $\left(\mathrm{E}_{z}\right)$ and the azimuthal $\left(\mathrm{H}_{\phi}\right)$ and radial $\left(\mathrm{H}_{r}\right)$ components of the magnetic fields resulting from lightning strikes to the $\mathrm{CN}$ Tower have been recorded. Because the new LeCroy LT342L digitizer which was installed in 2002 only has two channels, it was recently decided to only measure the vertical electric field and the azimuthal magnetic field, since the radial magnetic field is usually very low. In the summer of 2004 , the sensors were moved from the roof of the Rosebrugh building at the University of Toronto to the roof of the Pratt building at the University of Toronto. This move had to occur because the laboratory was obligated to move from Rosebrugh to Pratt, however, the Pratt building is only a few tens of meters to the west of the Rosebrugh 
building, making the laboratory still approximately $2 \mathrm{~km}$ north of the CN Tower.

The electric field sensor is an active hollow hemispherical shaped monopole with a sensitivity of $1.44 \mathrm{mV} /(\mathrm{V} / \mathrm{m})[49]$. The sensor has low and high $3 \mathrm{~dB}$ roll off frequencies of $47 \mathrm{~Hz}$ and $100 \mathrm{MHz}$, respectively [49]. The azimuthal and radial magnetic field sensors are small loop antenna type and have sensitivities of $166 \mathrm{mV} /(\mathrm{A} / \mathrm{m})$ each and $3 \mathrm{~dB}$ bandwidths of $635 \mathrm{~Hz}$ to $134 \mathrm{MHz}$ and $697 \mathrm{~Hz}$ to $150 \mathrm{MHz}$, respectively [49]. Figure 4.1 .3 shows a picture of the azimuthal magnetic field sensor being installed on the University of Toronto Pratt building rooftop. The azimuthal magnetic field sensor was installed so the CN Tower is in the plane of its circular loop.

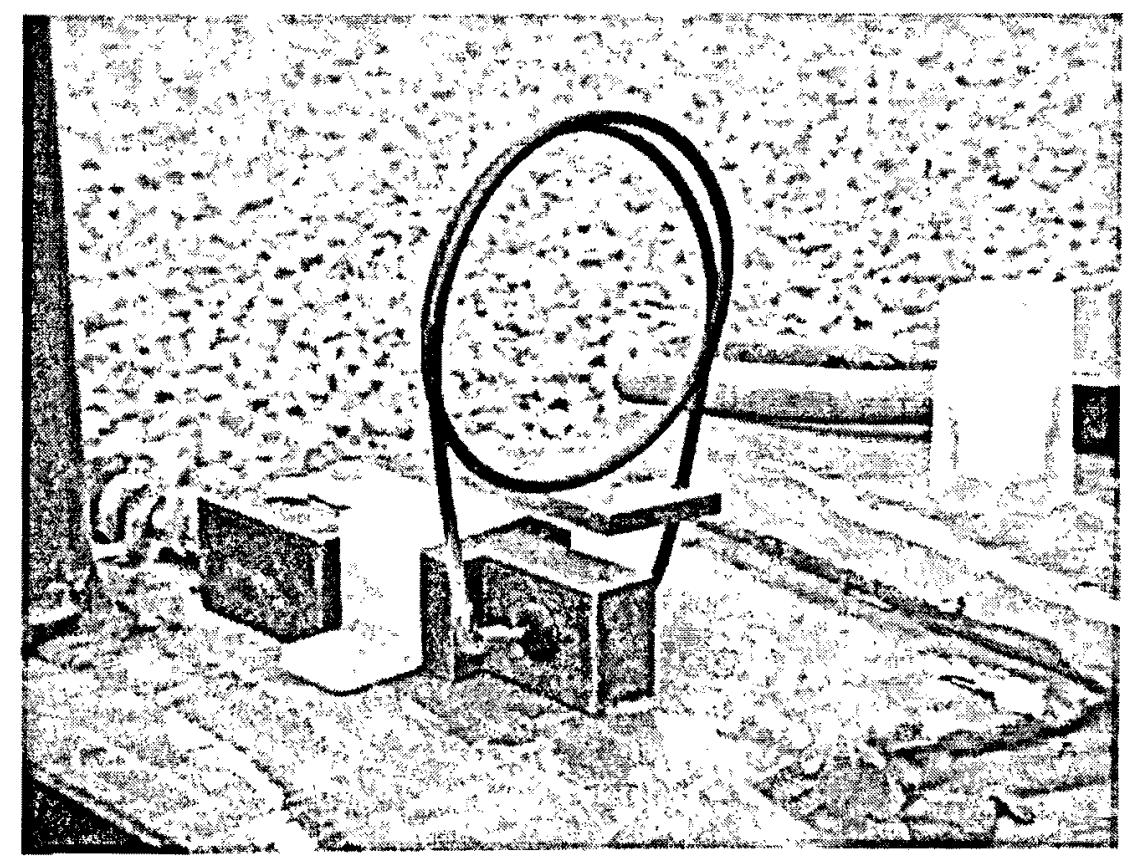

Figure 4.1.3: The azimuthal magnetic field sensor.

The electric and magnetic field sensors are connected via $50 \Omega$ coaxial cable to the LeCroy LT342L ( 8 bit, 2 ns) digitizer. Currently the electric field sensor is being used in channel 2 to trigger the digitizer. Similar to the CN Tower installation, when the digitizer is triggered it will send a pulse and trigger the Truetime 560-5908 PCI-SG2 GPS timing system, which 
will immediately record the GPS time with an accuracy of $1 \mu \mathrm{s}$. The overall risetime of the field system is estimated as being less than 5 ns, so the GPS time accuracy is not affected.

\subsubsection{Summary}

A summary of the the CN Tower lightning measurement systems is given below:

\section{- Measurement Systems at the CN Tower}

- Current Derivative Measurement System

* $3 \mathrm{~m}, 40 \mathrm{MHz}$ Rogowski current sensing coil - $474 \mathrm{~m}$ above ground level

* $6 \mathrm{~m}, 20 \mathrm{MHIz}$ Rogowski current sensing coil (noise protected) - $509 \mathrm{~m}$ above ground level

- Tructime 560-5908 PCI-SG2 GPS system for time synchronization (1 $\mu$ s accurate)

- Measurement Systems at the University of Toronto Pratt Building Lightning Laboratory (located approximately $2 \mathrm{~km}$ north of the CN Tower)

- Electric and Magnetic Field Measuring systems

* Electric field sensor - measuring the vertical component $\left(\mathrm{E}_{z}\right)$

* Magnetic field sensor - measuring the azimuthal $\left(\mathrm{H}_{\phi}\right)$ component

- Video Recording Systems

* Phantom V2.0 1000 frame/sec (1 ms resolution) digital High-Speed Camera

* Video Camera (VIIS and DVD) recording system (16 ms resolution) with Truetime XL-DC GPS timing (1 ms accurate)

- Tructime 500-5908 PCI-SG2 GPS system for time synchronization ( $1 \mu$ s accurate)

As previously mentioned, the NALDN measures and provides the following lightning information: location of stroke, time of stroke, polarity of stroke, peak current of stroke and confidence ellipse $[24,29]$. The $\mathrm{CN}$ Tower instrumentation listed above can be used in the following fashion to obtain data for the following NALDN measurements: 
- location of stroke

- The current derivative measurement system can identify the individual strokes within a $\mathrm{CN}$ Tower lightning flash and obviously verify the location of the stroke. The video recording systems can also verify the location of the lightning flash.

- time of stroke

- The GPS time synchronization system can verify the time of each stroke from the current derivative measurement system at the $\mathrm{CN}$ Tower and from the field measurement system located $2 \mathrm{~km}$ north of the $\mathrm{CN}$ Tower. The video recording (VHS and DVD) system also has GPS time synchronization, and can verify the time of a lightning flash.

- polarity of stroke

- The current derivative and the electric field measurement system can verify the polarity of each stroke.

- peak current of stroke

- The current derivative measuring system can provide the peak current of each stroke.

- confidence ellipse

- By knowing the exact location and time of a stroke as described above, the confidence ellipse provided by the NALDN can be verified to see if the location accuracy model used in the network is accurate.

Therefore, the CN Tower provides an excellent opportunity to evaluate every single measurement provided by the NALDN. The only other evaluation which was capable of doing this was the rocket-triggered evaluation performed in Florida at Camp Blanding in [8]. The flash detection efficiency, stroke detection efficiency, location accuracy, peak current estimation and confidence ellipses are evaluated in the next section using $\mathrm{CN}$ Tower lightning data from 2005. 


\subsection{North American Lightning Detection Network Performance Analysis}

The recently installed GPS timing system at the CN Tower recorded the lightning stroke time with an accuracy of $1 \mu \mathrm{s}$ for the data acquired in 2005. The North American Lightning Detection Network (NALDN) provided stroke data that is precise and accurate to $1 \mathrm{~ms}$ and contains the following information: latitude and longitude, signal strength (which is multiplied by 0.185 to obtain peak current as shown in Equation 3.4.4), 50\% error ellipse semi-major axis length, the error ellipse angle of orientation and eccentricity, and the $\chi^{2}$ value. The CN Tower stroke data was matched to the NALDN data with a perfect $1 \mathrm{~ms}$ accuracy for each stroke.

Table 4.2.1 provides an analysis of the 2005 data. The table shows NALDN absolute location accuracy for the strokes detected, as well as the estimated peak current. For comparison, the $\mathrm{CN}$ Tower peak current and current wavefront maximum steepness are shown. As mentioned in Section 4.1.4, the field measurement system located $2 \mathrm{~km}$ north of the CN Tower at the University of Toronto Pratt building Lightning Laboratory also contains a GPS timing system accurate to $1 \mu \mathrm{s}$, allowing these field measurements to be accurately matched. The peak of the azimuthal component of the magnetic field $\left(\mathrm{H}_{\phi}\right)$ and the peak of the vertical component of the electric field $\left(\mathrm{E}_{z}\right)$ for each stroke are also listed in Table 4.2.1. The digital video recording system located at the Lightning Laboratory also has GPS timing accurate to $1 \mathrm{~ms}$, allowing for the visual confirmation of each lightning event. It should be noted that only the flash time is provided for each event and that the NALDN predicted latitude and longitude for each stroke are not listed, in order to avoid publicly disclosing restricted NALDN data, as well as CN Tower data. 


\begin{tabular}{|c|c|c|c|c|c|c|c|c|}
\hline Date & $\begin{array}{l}\text { Flash } \\
\text { Time } \\
\text { (UTC) }\end{array}$ & $\begin{array}{l}\text { Stroke } \\
\text { No. }\end{array}$ & $\begin{array}{l}\text { NALDN } \\
\text { Absolute } \\
\text { Location } \\
\text { Error } \\
(\mathrm{km})\end{array}$ & $\begin{array}{l}\text { CN } \\
\text { Tower } \\
\text { Peak } \\
\text { Current } \\
\text { (kA) }\end{array}$ & $\begin{array}{l}\text { NALDN } \\
\text { Peak } \\
\text { Current } \\
\text { (kA) }\end{array}$ & $\begin{array}{l}\text { CN Tower } \\
\text { Current } \\
\text { Wavefront } \\
\text { Maximum } \\
\text { Steepness } \\
(\mathrm{kA} / \mathrm{s})\end{array}$ & $\begin{array}{l}\text { Peak } \\
\text { Azimuthal } \\
\text { Magnetic } \\
\text { Field } H_{\phi} \\
(\mathrm{A} / \mathrm{m})\end{array}$ & $\begin{array}{l}\text { Peak } \\
\text { Electric } \\
\text { Field } \\
\text { E }_{z} \\
(\mathrm{~V} / \mathrm{m})\end{array}$ \\
\hline $06 / 15 / 05$ & $04: 31: 38$ & 1 & 0.132 & -21 & -47.2 & -6 & - & - \\
\hline $08 / 19 / 05$ & $11: 57: 14$ & $\begin{array}{l}1 \\
2\end{array}$ & . & $\begin{array}{l}\text { low } \\
\text { low }\end{array}$ & - & $\begin{array}{l}\text { low } \\
\text { low }\end{array}$ & - & - \\
\hline $08 / 19 / 05$ & $12: 56: 35$ & $\begin{array}{l}1 \\
2 \\
3 \\
4\end{array}$ & $\begin{array}{l}- \\
- \\
-\end{array}$ & $\begin{array}{l}\text { low } \\
\text { low } \\
\text { low } \\
\text { low }\end{array}$ & $\begin{array}{l}- \\
- \\
-\end{array}$ & $\begin{array}{l}\text { low } \\
\text { low } \\
\text { low } \\
\text { low }\end{array}$ & $\begin{array}{l}- \\
- \\
- \\
-\end{array}$ & $\begin{array}{l}- \\
- \\
-\end{array}$ \\
\hline $08 / 19 / 05$ & $17: 55: 56$ & $\begin{array}{l}1 \\
2 \\
3 \\
4 \\
5 \\
6 \\
7 \\
8 \\
9 \\
10 \\
11\end{array}$ & $\begin{array}{l}- \\
- \\
0.465 \\
0.390 \\
0.263 \\
- \\
- \\
- \\
- \\
- \\
-\end{array}$ & $\begin{array}{l}\text { low } \\
-2.5 \\
-5.5 \\
-4.7 \\
-4.5 \\
-2.25 \\
-2.75 \\
-2 \\
-2 \\
-3 \\
-3\end{array}$ & $\begin{array}{l}- \\
- \\
-22.4 \\
-22.2 \\
-19.3 \\
- \\
- \\
- \\
- \\
- \\
-\end{array}$ & $\begin{array}{l}\text { low } \\
-4.2 \\
-27.5 \\
-26 \\
-24 \\
-14.5 \\
-20 \\
-13.5 \\
-12 \\
-26 \\
-17.5\end{array}$ & $\begin{array}{l}- \\
0.75 \\
1.5 \\
1.3 \\
1.25 \\
0.7 \\
0.84 \\
0.69 \\
0.65 \\
0.9 \\
0.99\end{array}$ & $\begin{array}{l}- \\
350 \\
700 \\
600 \\
550 \\
300 \\
375 \\
290 \\
280 \\
410 \\
490\end{array}$ \\
\hline $08 / 19 / 05$ & $18: 11: 41$ & $\begin{array}{l}1 \\
2 \\
3 \\
4 \\
5 \\
6 \\
7 \\
8 \\
9 \\
10 \\
11\end{array}$ & $\begin{array}{l}- \\
0.465 \\
1.214 \\
0.263 \\
0.368 \\
- \\
0.369 \\
0.295 \\
- \\
- \\
-\end{array}$ & $\begin{array}{l}\text { low } \\
-5.8 \\
-3.75 \\
-5.1 \\
-4.6 \\
-2.5 \\
-5.5 \\
\text { low } \\
-2.8 \\
-3.8 \\
-2\end{array}$ & $\begin{array}{l}- \\
-29.7 \\
-12.7 \\
-26.2 \\
-22.6 \\
- \\
-26.7 \\
-22.1 \\
- \\
- \\
-\end{array}$ & $\begin{array}{l}\text { low } \\
-22 \\
-17 \\
-27.5 \\
-27.5 \\
-18 \\
-23 \\
\text { low } \\
-27.5 \\
-22.5 \\
-27.5\end{array}$ & $\begin{array}{l}- \\
1.49 \\
1.07 \\
1.5 \\
1.4 \\
0.75 \\
1.49 \\
1.47 \\
0.89 \\
1.1 \\
0.68\end{array}$ & $\begin{array}{l}- \\
700 \\
480 \\
660 \\
625 \\
350 \\
650 \\
750 \\
390 \\
550 \\
310\end{array}$ \\
\hline $08 / 19 / 05$ & $18: 13: 13$ & $\begin{array}{l}1 \\
2 \\
3 \\
4 \\
5 \\
6 \\
7\end{array}$ & $\begin{array}{l}0.475 \\
- \\
0.263 \\
- \\
- \\
- \\
-\end{array}$ & $\begin{array}{l}-6.5 \\
-3.9 \\
-7.9 \\
-3.4 \\
-3 \\
-1.34 \\
-2.75\end{array}$ & $\begin{array}{l}-35.5 \\
- \\
-43.8 \\
- \\
- \\
- \\
- \\
\end{array}$ & $\begin{array}{l}-22.5 \\
-20 \\
-26 \\
-25 \\
-25 \\
-2.25 \\
-17.5 \\
\end{array}$ & $\begin{array}{l}1.7 \\
1.05 \\
2.3 \\
1.07 \\
0.96 \\
- \\
0.95\end{array}$ & $\begin{array}{l}850 \\
500 \\
1150 \\
475 \\
410 \\
- \\
460 \\
\end{array}$ \\
\hline $08 / 19 / 05$ & $18: 37: 08$ & 1 & 0.578 & -4.7 & -19.7 & -20.5 & 1.22 & 550 \\
\hline
\end{tabular}


(Table continued from previous page)

\begin{tabular}{|l|l|l|l|l|l|l|l|l|}
\hline Date & $\begin{array}{l}\text { Flash } \\
\text { Time } \\
\text { (UTC) }\end{array}$ & $\begin{array}{l}\text { Stroke } \\
\text { No. }\end{array}$ & $\begin{array}{l}\text { NALDN } \\
\text { Absolute } \\
\text { Location } \\
\text { Error } \\
(\mathbf{k m})\end{array}$ & $\begin{array}{l}\text { CN } \\
\text { Tower } \\
\text { Peak } \\
\text { Current } \\
(\mathrm{kA})\end{array}$ & $\begin{array}{l}\text { NALDN } \\
\text { Peak } \\
\text { Current } \\
(\mathbf{k A})\end{array}$ & $\begin{array}{l}\text { CN Tower } \\
\text { Current } \\
\text { Wavefront } \\
\text { Maximum } \\
\text { Steepness } \\
(\mathrm{kA} / \mathrm{s})\end{array}$ & $\begin{array}{l}\text { Peak } \\
\text { Azimuthal } \\
\text { Magnetic } \\
\text { Field } \mathbf{H}_{\phi} \\
(\mathbf{A} / \mathrm{m})\end{array}$ & $\begin{array}{l}\text { Peak } \\
\text { Electric } \\
\text { Field } \\
\mathbf{E}_{\boldsymbol{z}} \\
(\mathbf{V} / \mathbf{m})\end{array}$ \\
\hline $08 / 19 / 05$ & $18: 37: 52$ & 1 & 0.379 & -3.85 & -22.4 & -11.5 & 1.09 & 520 \\
& & 2 & 0.304 & -4.5 & -20.7 & -20.5 & 1.32 & 620 \\
& & 3 & 0.132 & -8 & -44.1 & -10 & 2.35 & 1100 \\
& & 4 & 0.354 & -5.2 & -21.7 & -22 & 1.45 & 650 \\
\hline $08 / 19 / 05$ & $19: 26: 00$ & 1 & 0.354 & -5.25 & -31.9 & -9.6 & 1.3 & 650 \\
& & 2 & 0.095 & -3.5 & -18.6 & -25 & 0.99 & 475 \\
& & 3 & 0.358 & -4.25 & -21.1 & -23 & 1.06 & 525 \\
& & 4 & 0.304 & -4 & -20.7 & -16 & 1.1 & 550 \\
& & 5 & - & -2.1 & - & -11 & 0.65 & 265 \\
& & 6 & 0.759 & -3.78 & -13.8 & -21 & 0.98 & 460 \\
\hline
\end{tabular}

Table 4.2.1: NALDN data Analysis using CN Tower lightning data from 2005.

In Table 4.2.1, the flash time is given in Coordinated Universal Time (UTC), formerly known as Greenwich Mean Time (GMT). A '-' is displayed if the NALDN or the field measurement system $2 \mathrm{~km}$ north of the CN Tower did not detect a stroke. The 'low' message is displayed for events which contained a very low current peak or current wavefront maximum steepness. It is believed that these events are lightning M-components. A negative value for peak current and current wavefront maximum steepness indicates that the stroke lowered negative charge to ground, as demonstrated by the corresponding positive value for the vertical component of the electric field $\left(E_{z}\right)$. 
The following table provides a summary of the NALDN performance and includes all CN Tower lightning strokes or events in the data analysis.

\begin{tabular}{|l|l|l|l|l|l|l|l|}
\hline $\begin{array}{l}\text { Number } \\
\text { of } \\
\text { Flashes }\end{array}$ & $\begin{array}{l}\text { Number } \\
\text { of } \\
\text { NALDN } \\
\text { Detected } \\
\text { Flashes }\end{array}$ & $\begin{array}{l}\text { NALDN } \\
\text { Flash } \\
\text { Detection } \\
\text { Efficiency }\end{array}$ & $\begin{array}{l}\text { Number } \\
\text { of } \\
\text { Strokes }\end{array}$ & $\begin{array}{l}\text { Number } \\
\text { of } \\
\text { NALDN } \\
\text { Detected } \\
\text { Strokes }\end{array}$ & $\begin{array}{l}\text { NALDN } \\
\text { Stroke } \\
\text { Detection } \\
\text { Efficiency }\end{array}$ & $\begin{array}{l}\text { Median } \\
\text { Absolute } \\
\text { Location } \\
\text { Error } \\
\text { (km) }\end{array}$ & $\begin{array}{l}\text { Mean } \\
\text { Absolute } \\
\text { Location } \\
\text { Error } \\
\text { (km) }\end{array}$ \\
\hline 9 & 7 & $78 \%$ & 47 & 22 & $47 \%$ & 0.356 & 0.390 \\
\hline
\end{tabular}

Table 4.2.2: Summary of the total lightning events recorded at the CN Tower, along with the corresponding NALDN detection efficiencies and absolute location errors.

The following table provides a summary of the NALDN performance excluding the CN Tower lightning events thought to be M-components. Other evaluations, such as the rockettriggered evaluation performed at Camp Blanding, do not include M-components in the data set used for the performance evaluation, since these events are not normal cloud-to-ground lightning, but are perturbations or transient impulses superimposed on a continuing current $[50]$.

\begin{tabular}{|l|l|l|l|l|l|l|l|}
\hline $\begin{array}{l}\text { Number } \\
\text { of } \\
\text { Flashes }\end{array}$ & $\begin{array}{l}\text { Number } \\
\text { of } \\
\text { NALDN } \\
\text { Detected } \\
\text { Flashes }\end{array}$ & $\begin{array}{l}\text { NALDN } \\
\text { Flash } \\
\text { Detection } \\
\text { Efficiency }\end{array}$ & $\begin{array}{l}\text { Number } \\
\text { of } \\
\text { Strokes }\end{array}$ & $\begin{array}{l}\text { Number } \\
\text { of } \\
\text { NALDN } \\
\text { Detected } \\
\text { Strokes }\end{array}$ & $\begin{array}{l}\text { NALDN } \\
\text { Stroke } \\
\text { Detection } \\
\text { Efficiency }\end{array}$ & $\begin{array}{l}\text { Median } \\
\text { Absolute } \\
\text { Location } \\
\text { Error } \\
\text { (km) }\end{array}$ & $\begin{array}{l}\text { Mean } \\
\text { Absolute } \\
\text { Location } \\
\text { Error } \\
\text { (km) }\end{array}$ \\
\hline 7 & 7 & $100 \%$ & 39 & 22 & $56 \%$ & 0.356 & 0.390 \\
\hline
\end{tabular}

Table 4.2.3: Summary of the flashes and strokes recorded at the CN Tower, excluding the events thought to be M-components, along with the corresponding NALDN detection efficiencies and absolute location errors. 
The following figures from Figure 4.2.1 to Figure 4.2.5 are taken from the single stroke flash that occurred at 18:37:08 (UTC) on August 19, 2005. These figures display a complete set of data acquired for a typical stroke to the CN Tower. The data set contains the current derivative waveform, the current waveform, the azimuthal magnetic field $\left(\mathrm{H}_{\phi}\right)$ waveform and the vertical component of the electric field $\left(E_{z}\right)$ waveform. A still frame of the event from the digital video recording system is also shown.

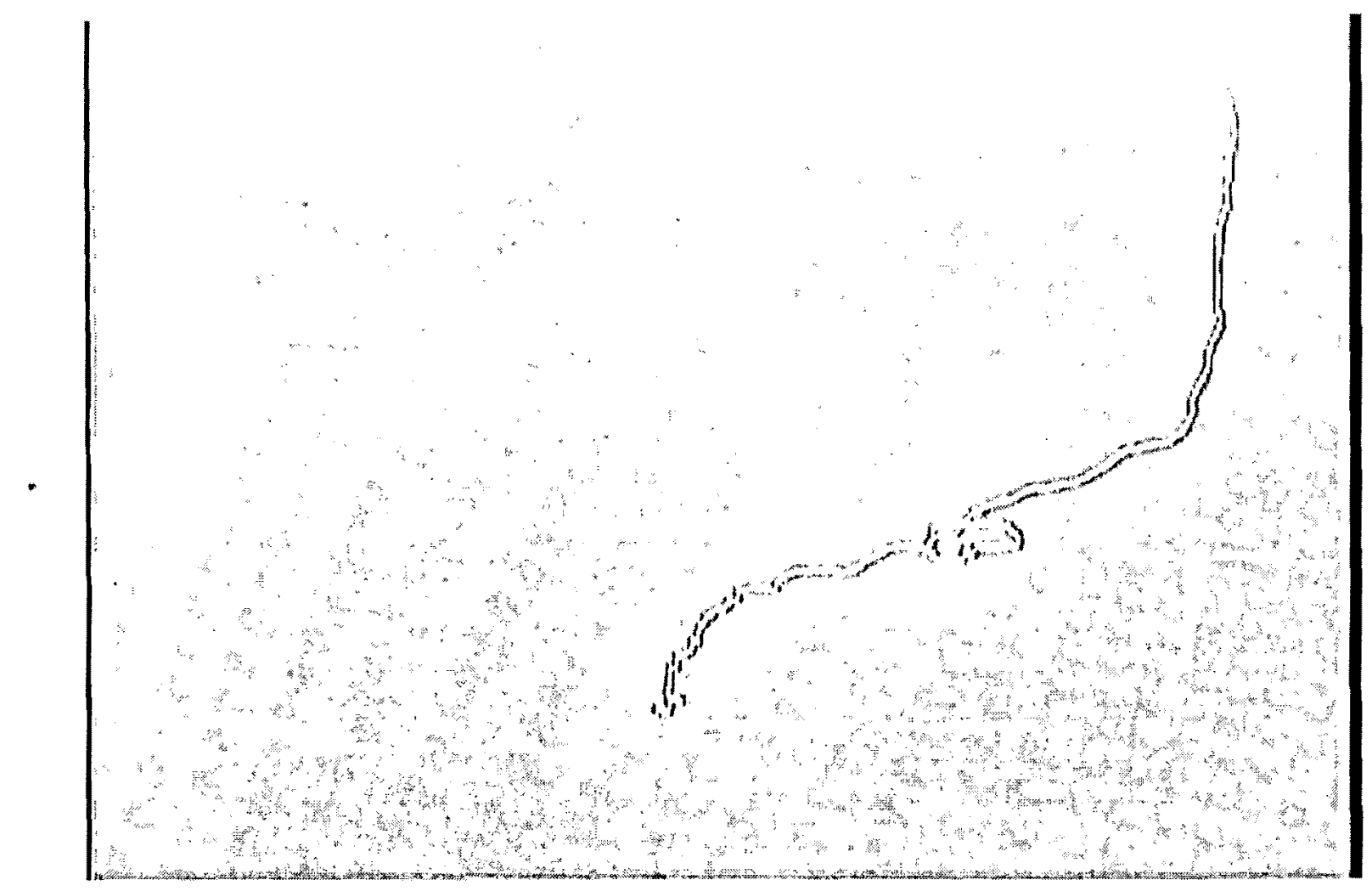

Figure 4.2.1: Digital video recording taken $2 \mathrm{~km}$ north of the CN Tower for the single stroke flash which occurred on August 19, 2005 at 18:37:08 (UTC). 
The following figures from Figure 4.2.1 to Figure 4.2.5 are taken from the single stroke flash that occurred at 18:37:08 (UTC) on August 19, 2005. These figures display a complete set of data acquired for a typical stroke to the $\mathrm{CN}$ Tower. The data set contains the current derivative waveform, the current waveform, the azimuthal magnetic field $\left(\mathrm{H}_{\phi}\right)$ waveform and the vertical component of the electric field $\left(\mathrm{E}_{z}\right)$ waveform. A still frame of the event from the digital video recording system is also shown.

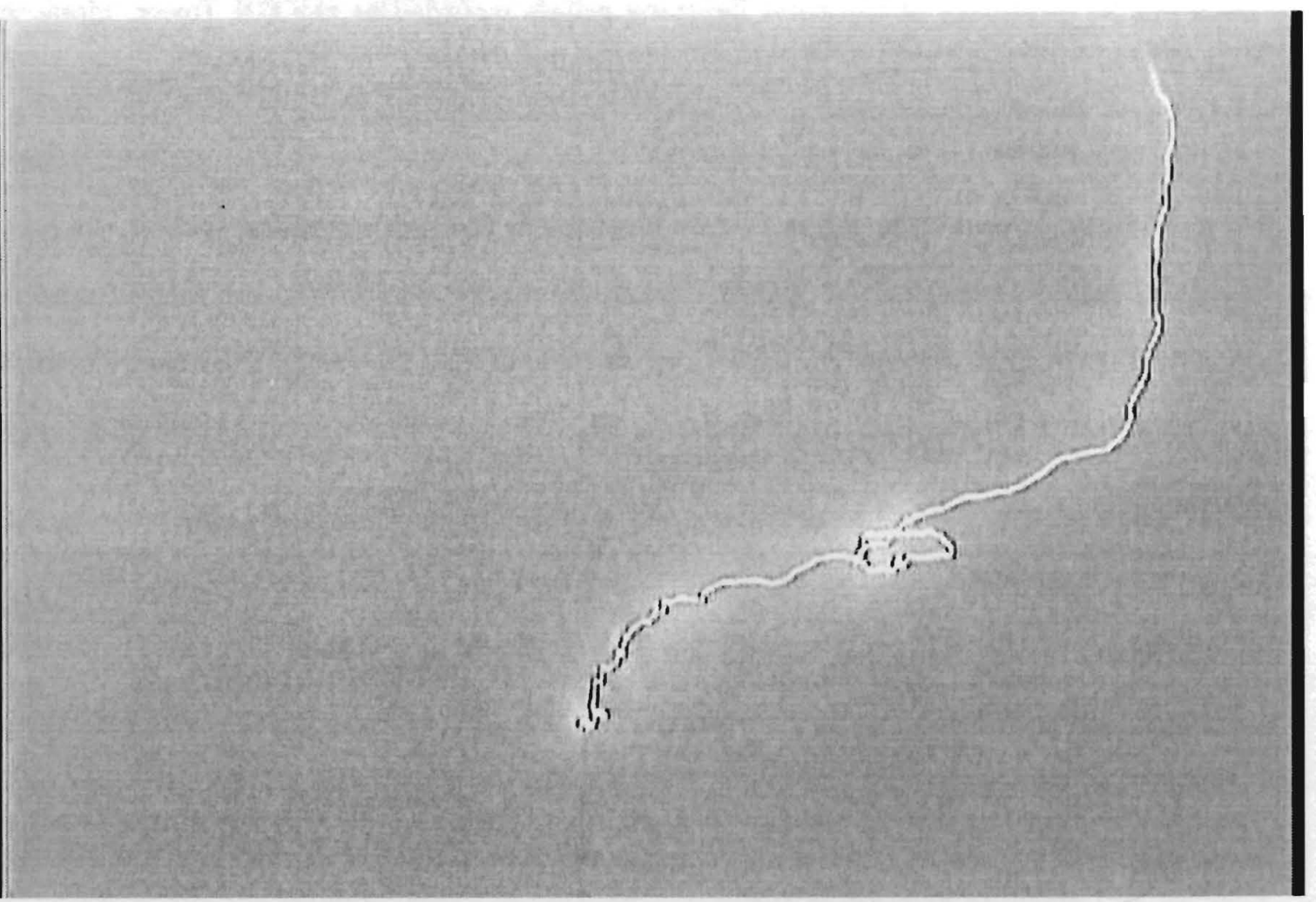

Figure 4.2.1: Digital video recording taken $2 \mathrm{~km}$ north of the CN Tower for the single stroke flash which occurred on August 19, 2005 at 18:37:08 (UTC). 


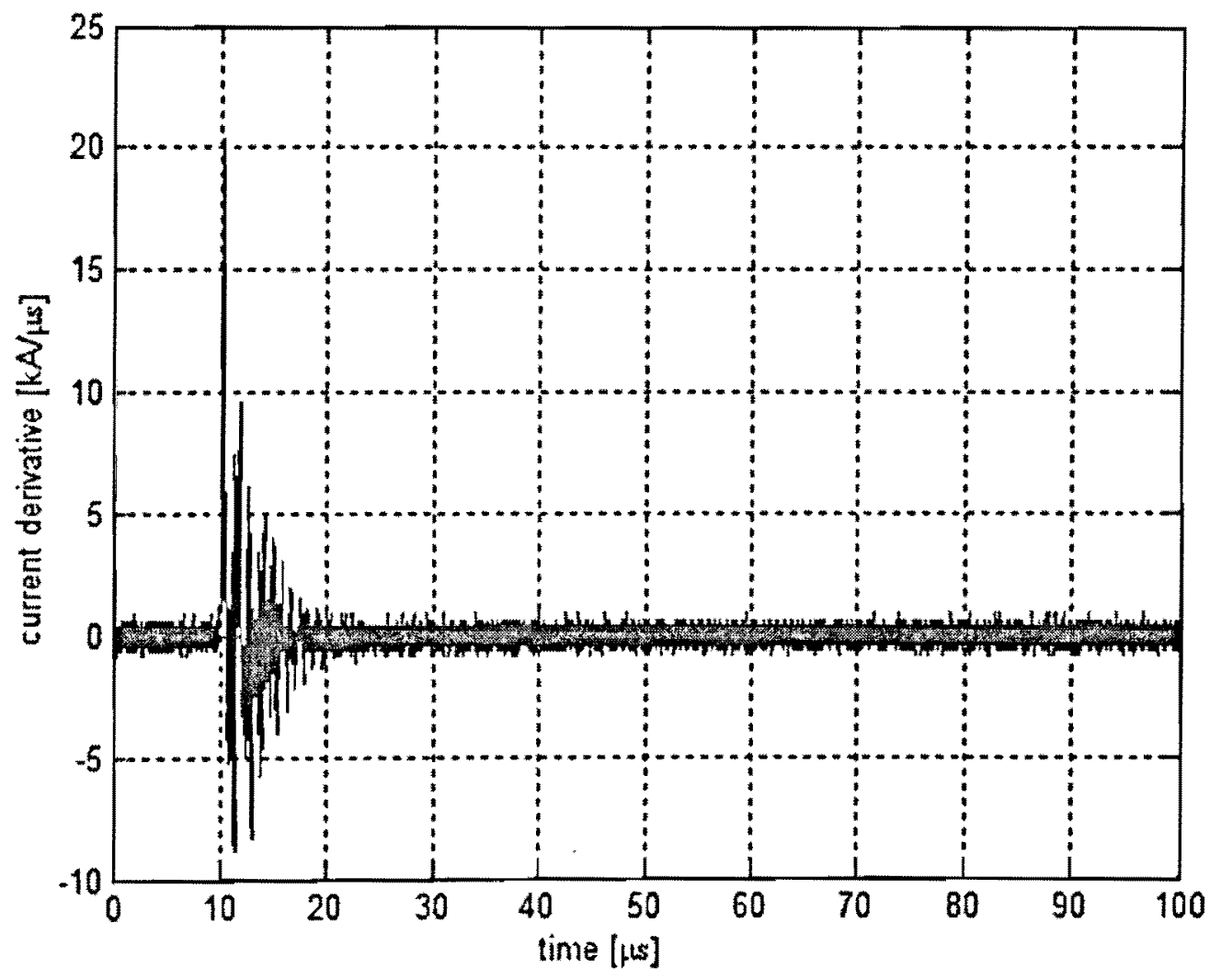

Figure 4.2.2: Current derivative waveform for the return stroke of a single stroke flash measured on August 19, 2005 at 18:37:08 (UTC). 


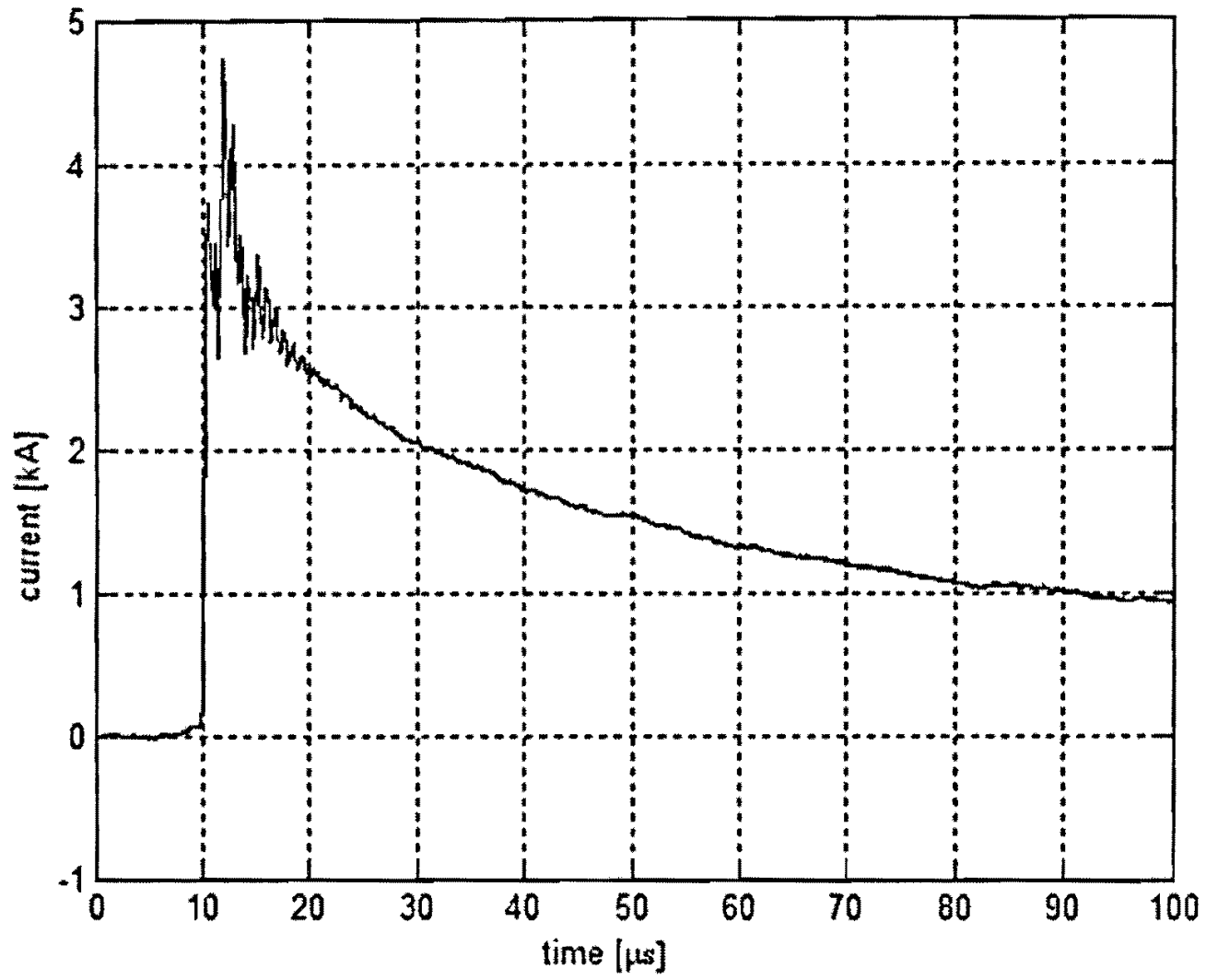

Figure 4.2.3: Current waveform for the single stroke flash which occurred on August 19, 2005 at 18:37:08 (UTC). This waveform was obtained by numerically integrating the signal of Figure 4.2.2. 


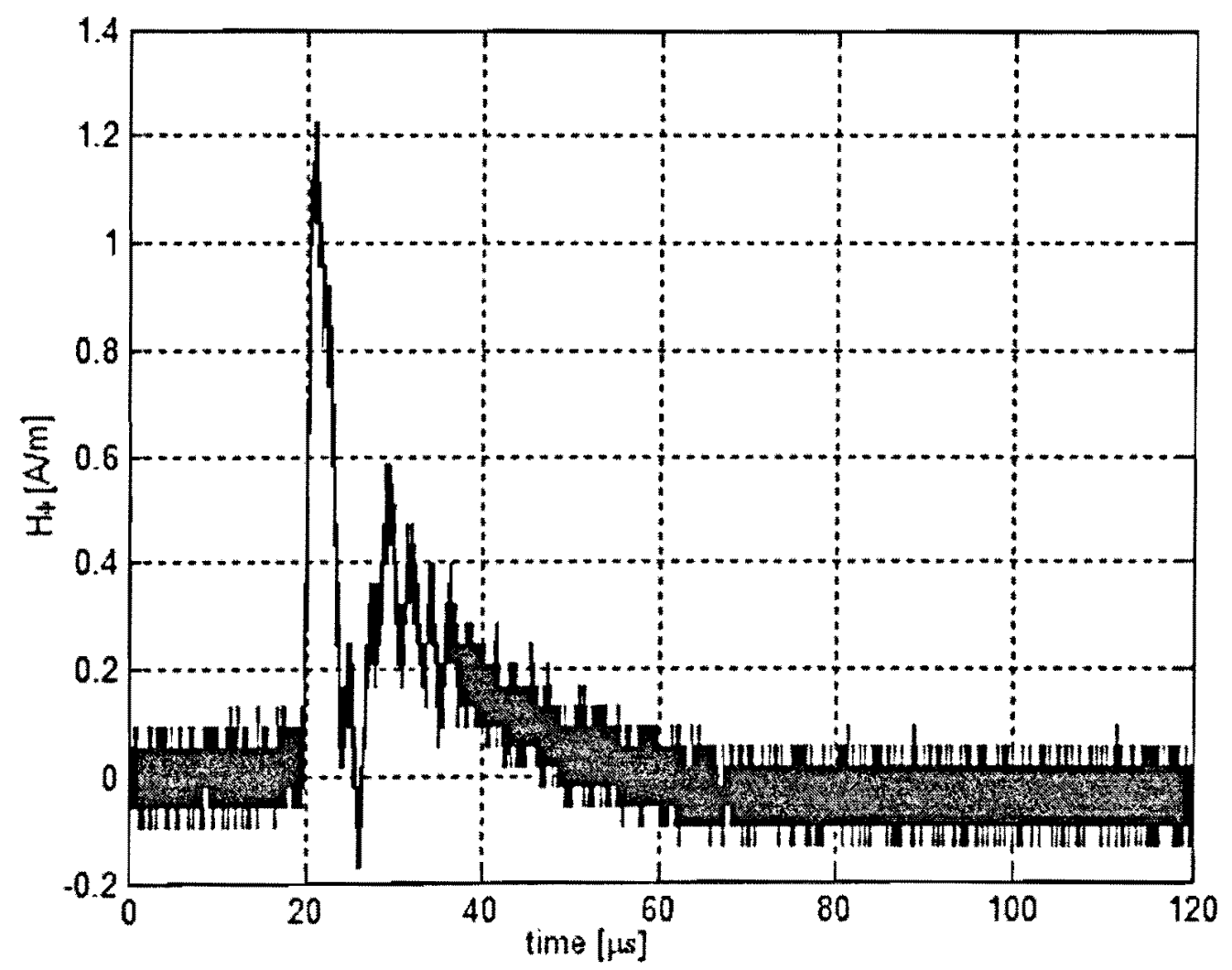

Figure 4.2.4: Azimuthal magnetic field $\left(\mathrm{H}_{\phi}\right)$ waveform observed $2 \mathrm{~km}$ north of the $\mathrm{CN}$ Tower for the return stroke of the single stroke flash measured on August 19, 2005 at 18:37:08 (UTC). 


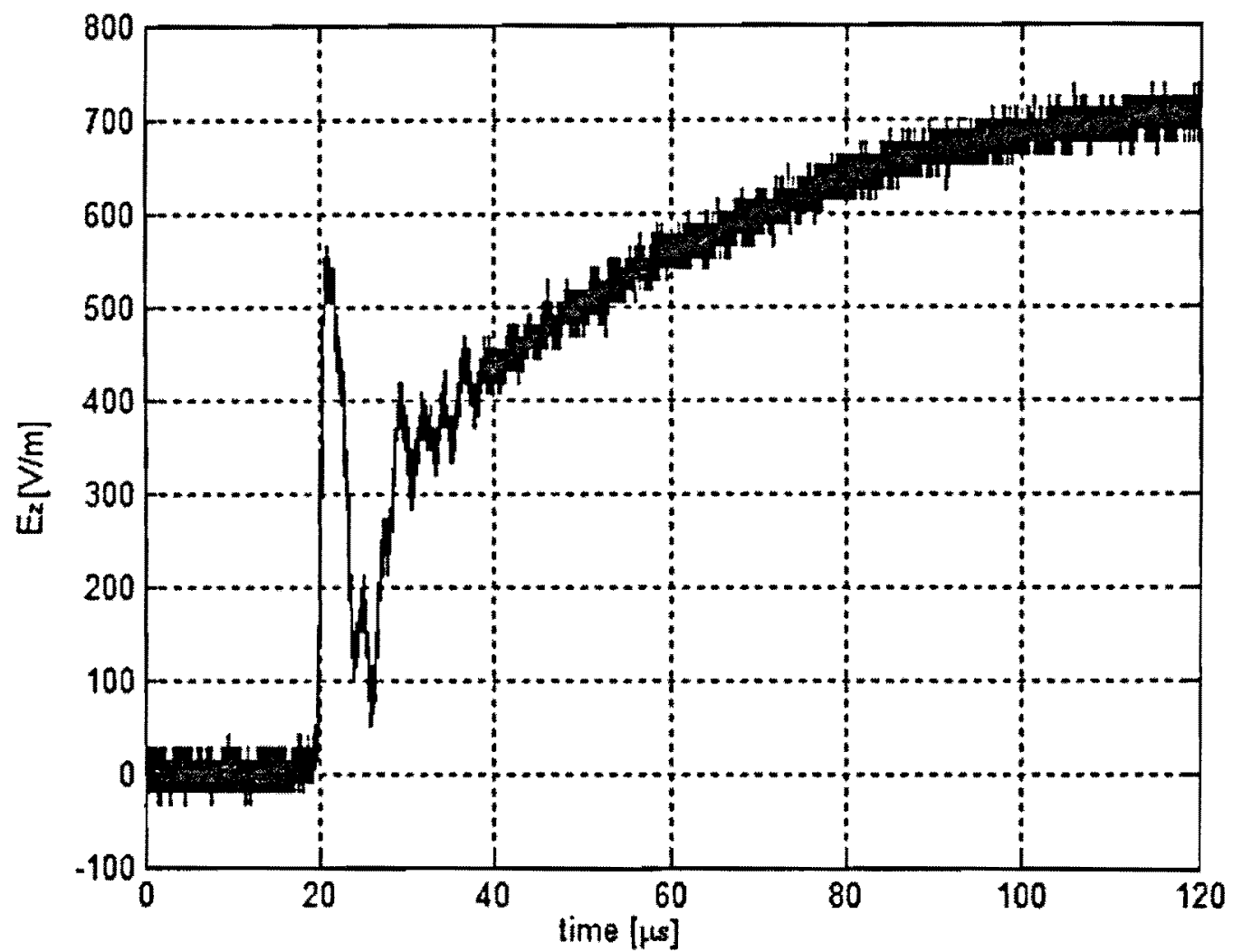

Figure 4.2.5: Vertical component of the electric field $\left(\mathrm{E}_{z}\right)$ waveform observed $2 \mathrm{~km}$ north of the CN Tower for the return stroke of the single stroke flash measured on August 19, 2005 at 18:37:08 (UTC). 
Table 4.2.4 shows the sensor configuration for the NALDN in the region around the CN Tower. As previously mentioned, the NALDN is composed of the U.S. National Lightning Detection Network (NLDN) and the Canadian Lightning Detection Network (CLDN). The upgraded IMPACT-ESP sensors from the NLDN play a large role in stroke detection for the region surrounding the $\mathrm{CN}$ Tower, since two of the closest three sensors to the $\mathrm{CN}$ Tower are NLDN IMPACT-ESP sensors.

\begin{tabular}{|l|l|}
\hline Sensor Type & $\begin{array}{l}\text { Distance from CN Tower } \\
(\mathbf{k m})\end{array}$ \\
\hline IMPACT-ESP & 66.8 \\
\hline LPATS-IV & 73.0 \\
\hline IMPACT-ESP & 286.1 \\
\hline LPATS-IV & 298.8 \\
\hline LPATS-IV & 304.8 \\
\hline IMPACT-ESP & 319.0 \\
\hline LPATS-IV & 327.9 \\
\hline IMPACT-ESP & 335.0 \\
\hline IMPACT-ESP & 337.9 \\
\hline IMPACT-ESP & 407.8 \\
\hline IMPACT-ESP & 471.6 \\
\hline LPATS-IV & 493.6 \\
\hline LPATS-IV & 512.2 \\
\hline
\end{tabular}

Table 4.2.4: NALDN sensor configuration in the region around the CN Tower, including the distance of each sensor from the $\mathrm{CN}$ Tower. The sensor location information was provided by Vaisala [51]. 
Figure 4.2.6 shows the locations of NLDN IMPACT-ESP and CLDN LPATS-IV sensors in the region around the CN Tower.

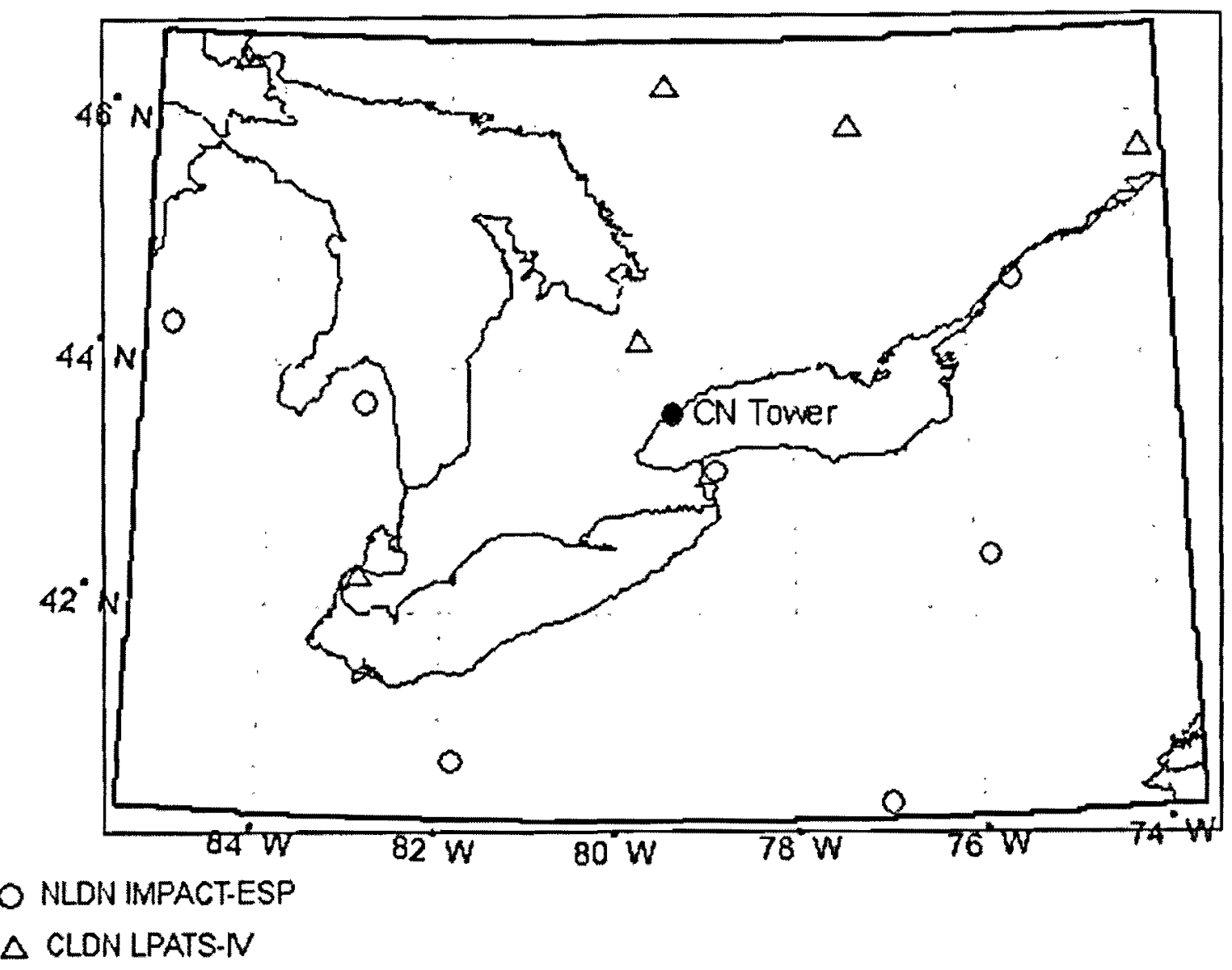

Figure 4.2.6: Map showing the locations of NLDN IMPACT-ESP and CLDN LPATS-IV sensors in the region around the $\mathrm{CN}$ Tower. The sensor location information was provided by Vaisala [51]. 


\subsubsection{Detection Efficiency}

Figure 4.2.7 shows a histogram of the NALDN stroke detection efficiency plotted as a function of current wavefront maximum steepness as measured at the CN Tower. Each column corresponds to a current wavefront maximum steepness range (bin size of $5 \mathrm{kA} / \mu \mathrm{s}$ ) and the ratio given inside each column indicates the number of strokes detected by the NALDN (numerator) and the number of strokes recorded at the CN Tower (denominator) for that range. The NALDN detected 22 out of the 47 strokes measured at the CN Tower. It should be noted that 8 out of the 11 strokes in the 0 to $5 \mathrm{kA} / \mu \mathrm{s}$ range are thought to be M-components.

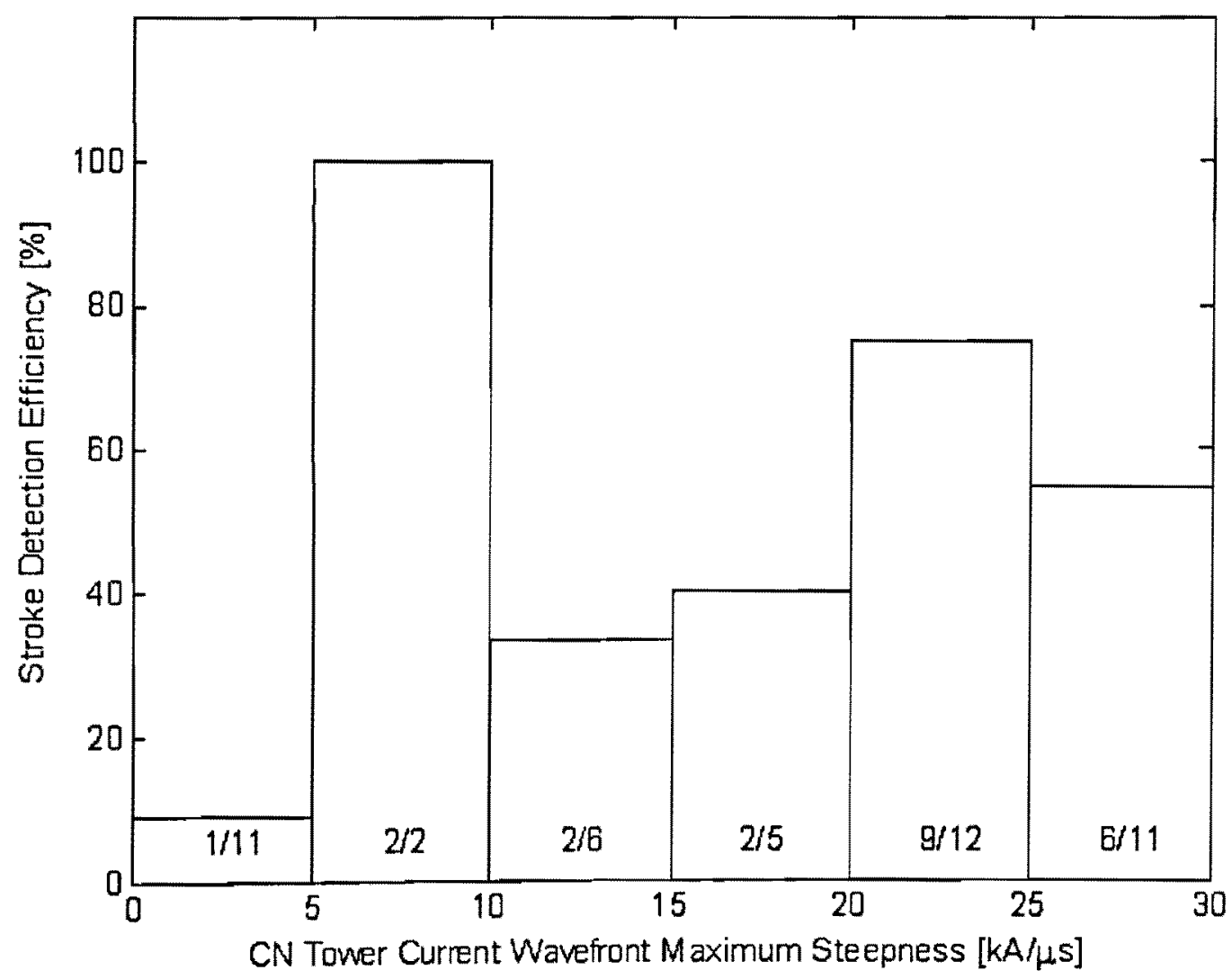

Figure 4.2.7: NALDN stroke detection efficiency plotted as a function of current wavefront maximum steepness as measured at the CN Tower. 
Figure 4.2 .8 shows a histogram of the NALDN stroke detection efficiency plotted as a function of peak current measured at the CN Tower. Each column corresponds to a peak current range (bin size of $1 \mathrm{kA}$ ) and the ratio given inside each column indicates the number of strokes detected by the NALDN (numerator) and the number of strokes recorded at the CN Tower (denominator), for that peak current range. The NALDN detected 22 out of the 47 strokes measured at the CN Tower. It should be noted that 8 out of the 9 strokes in the 0 to $1 \mathrm{kA}$ range are thought to be M-components.

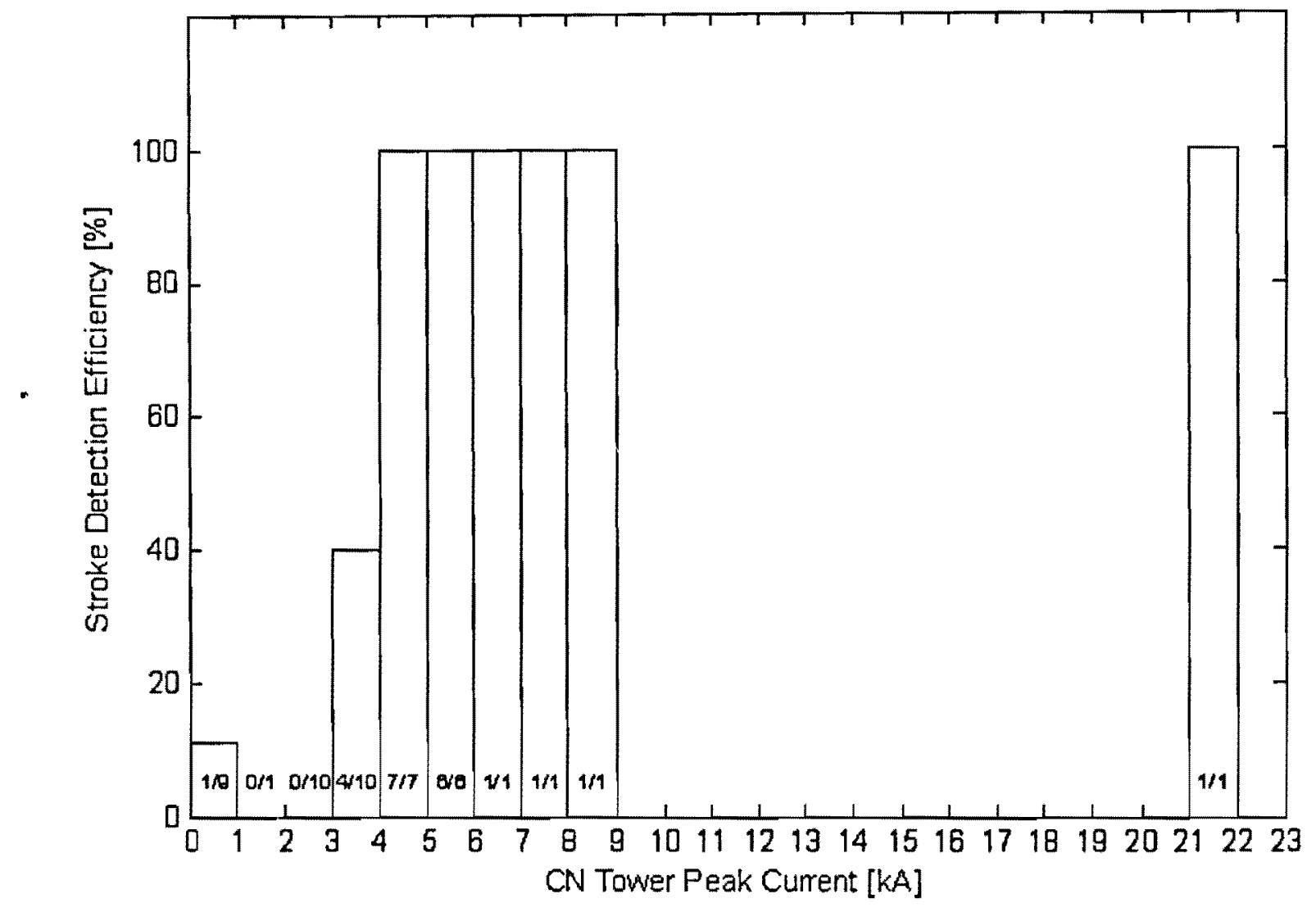

Figure 4.2.8: NALDN stroke detection efficiency plotted as a function of peak current measured at the CN Tower. 
Figure 4.2 .9 shows a histogram of the NALDN stroke detection efficiency plotted as a function of the peak of the azimuthal component of the magnetic field $\left(\mathrm{H}_{\phi}\right)$ measured 2 $\mathrm{km}$ north of the CN Tower. Each column corresponds to a peak field range (bin size of $0.2 \mathrm{~A} / \mathrm{m})$ and the ratio given inside each column indicates the number of strokes detected by the NALDN (numerator) and the number of strokes recorded by the field measurement equipment (denominator) for that range. The total number of azimuthal fields measured $2 \mathrm{~km}$ north of the CN Tower is 37 , out of which 21 were detected by the NALDN. The 8 strokes or events that are suspected of being M-components were not detected by the field measurement system and are therefore not part of this data set.

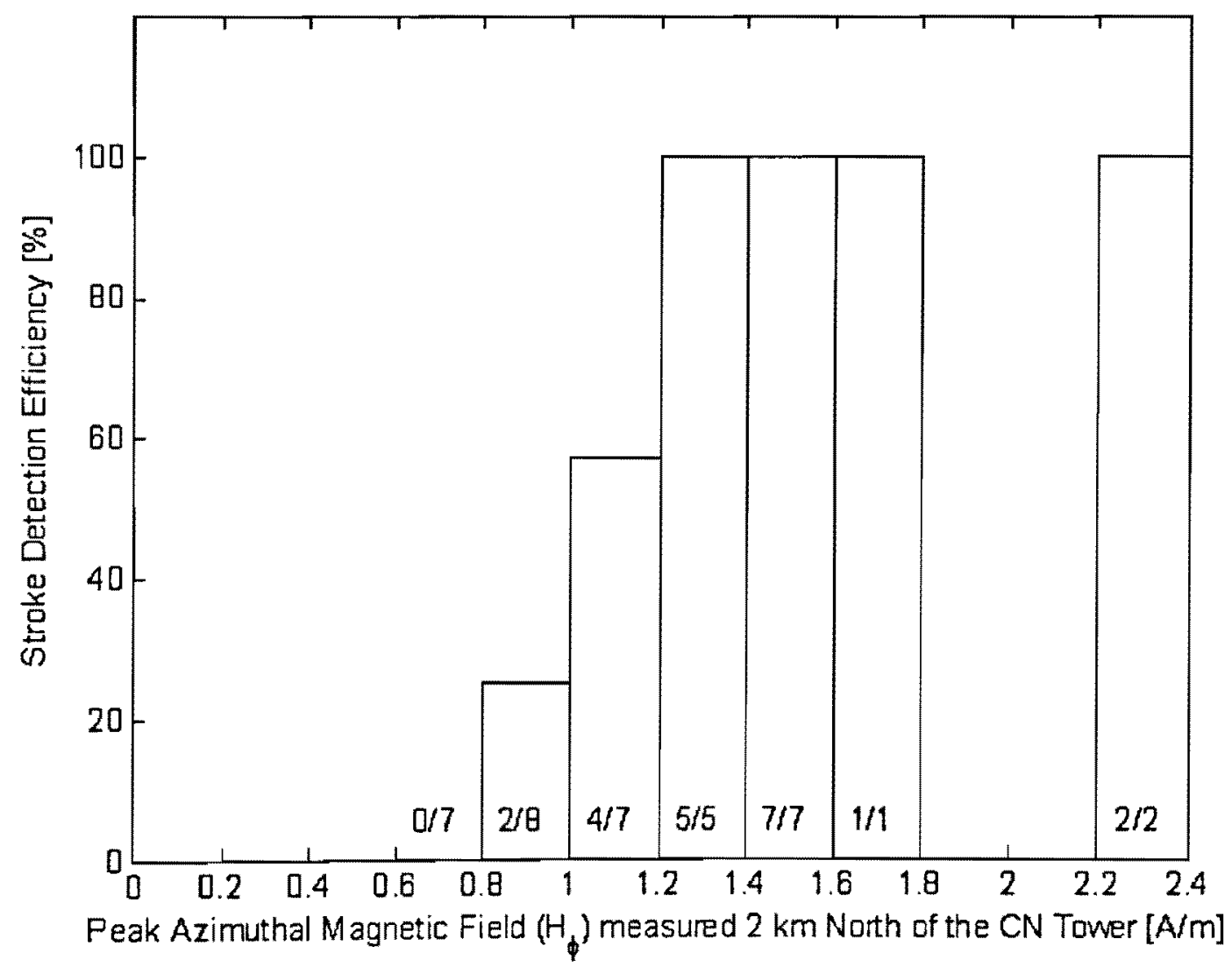

Figure 4.2.9: NALDN stroke detection efficiency plotted as a function of the peak of the azimuthal component of the magnetic field $\left(\mathrm{H}_{\phi}\right)$ measured $2 \mathrm{~km}$ north of the CN Tower. 
Figure 4.2.10 shows a histogram of the NALDN stroke detection efficiency plotted as a function of the peak of the vertical component of the electric field $\left(\mathrm{E}_{z}\right)$ measured $2 \mathrm{~km}$ north of the CN Tower. Each column corresponds to a peak field range (bin size of 50 $\mathrm{V} / \mathrm{m}$ ) and the ratio given inside each column indicates the number of strokes detected by the NALDN (numerator) and the number of strokes recorded by the field measurement equipment (denominator) for that range. The total number of fields measured $2 \mathrm{~km}$ north of the CN Tower is 37, out of which 21 were detected by the NALDN. The 8 strokes or events that are suspected of being M-components were not detected by the field measurement system and are therefore not part of this data set.

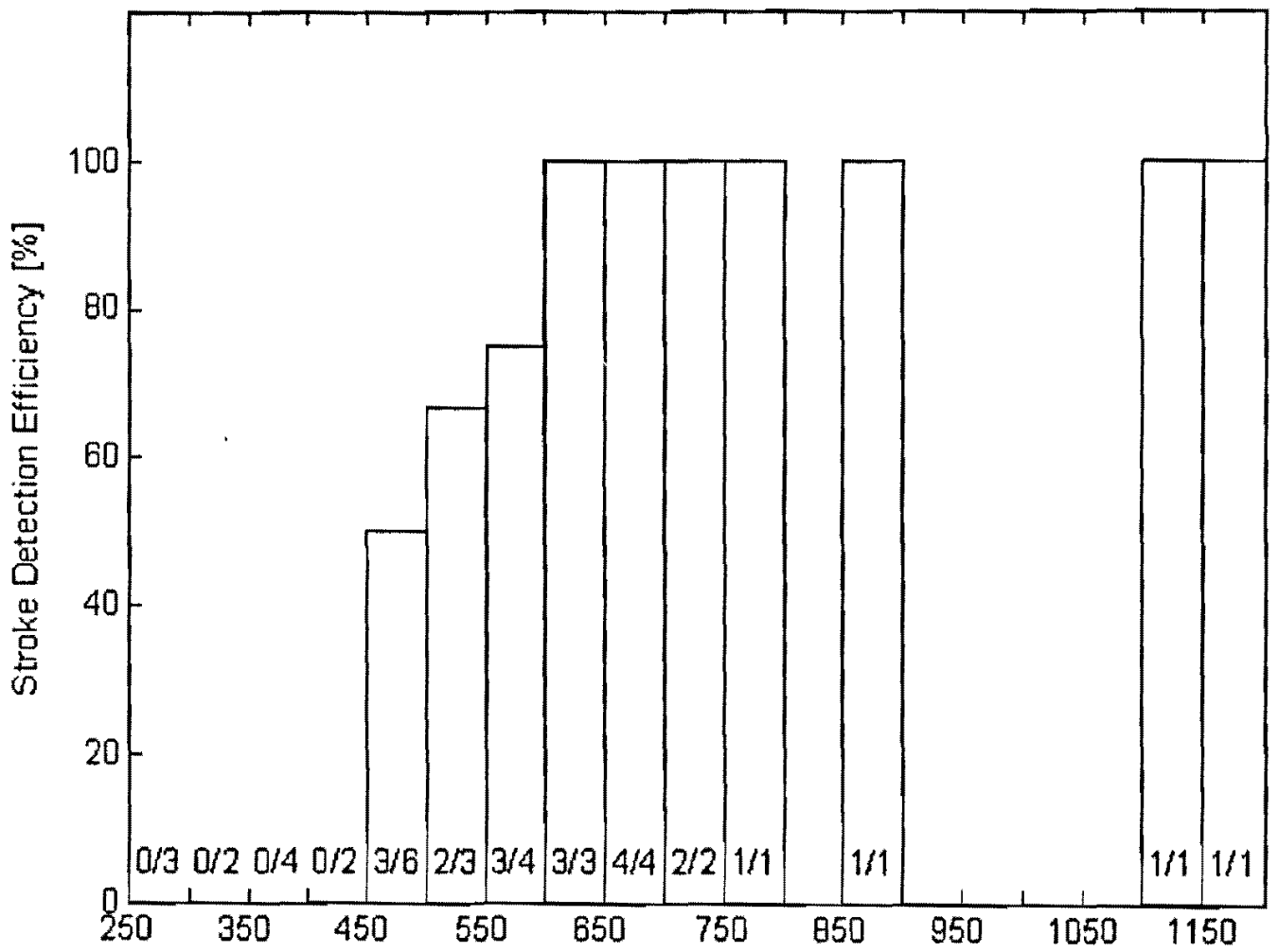

Peak Vertical Component of the Electric Field $\left(E_{2}\right)$ measured $2 \mathrm{~km}$ North of the $\mathrm{CN}$ Tower $[\mathrm{V} / \mathrm{m}]$

Figure 4.2.10: NALDN stroke detection efficiency plotted as a function of the peak of the vertical component of the electric field $\left(\mathrm{E}_{z}\right)$ measured $2 \mathrm{~km}$ north of the $\mathrm{CN}$ Tower. 


\subsubsection{Location Accuracy}

The following figures from Figure 4.2.11 to Figure 4.2.13 display the NALDN stroke location errors for the 22 detected strokes in 7 flashes.

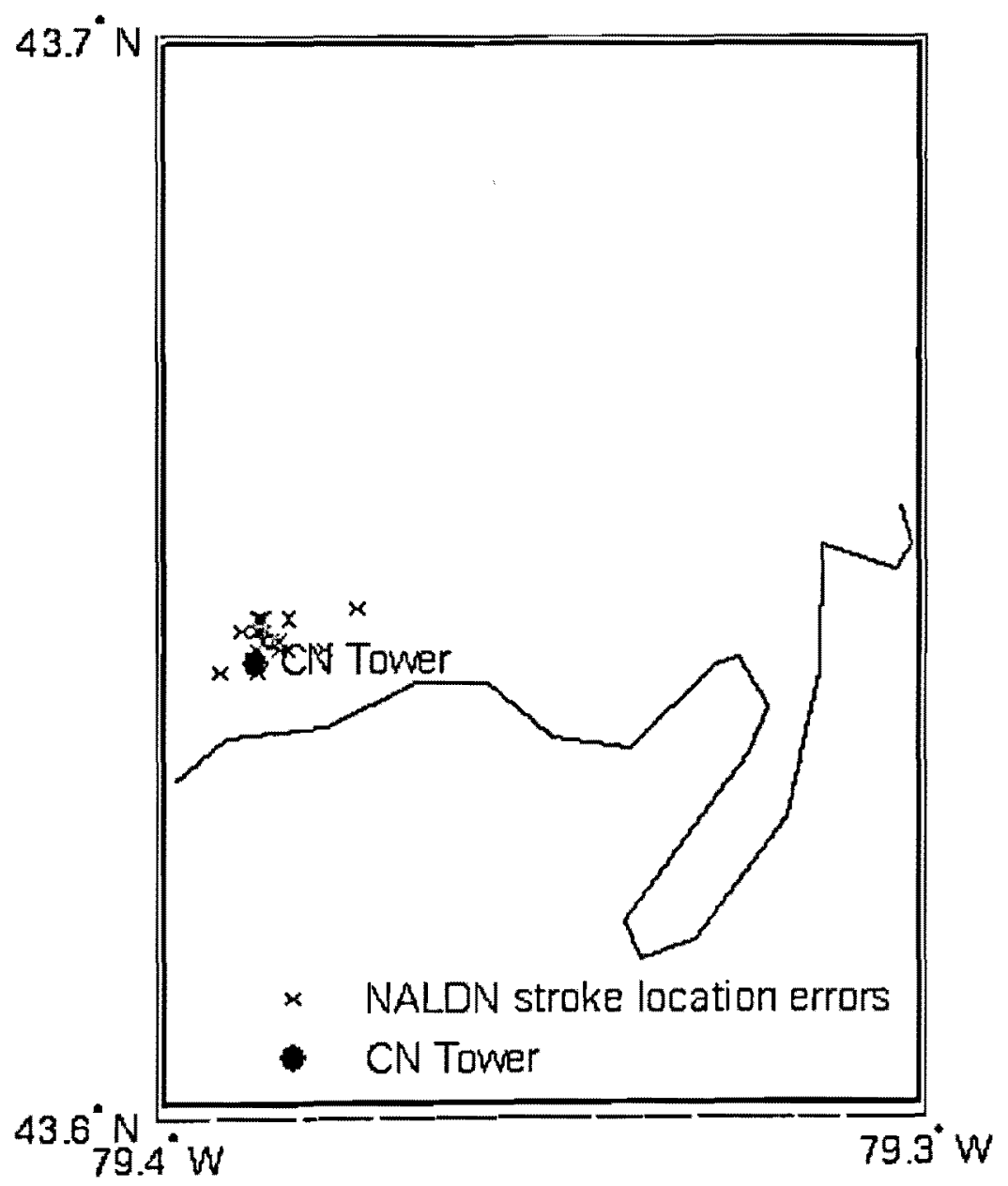

Figure 4.2.11: A distant latitude verses longitude view of the NALDN stroke location errors for the 22 detected strokes in 7 flashes. 


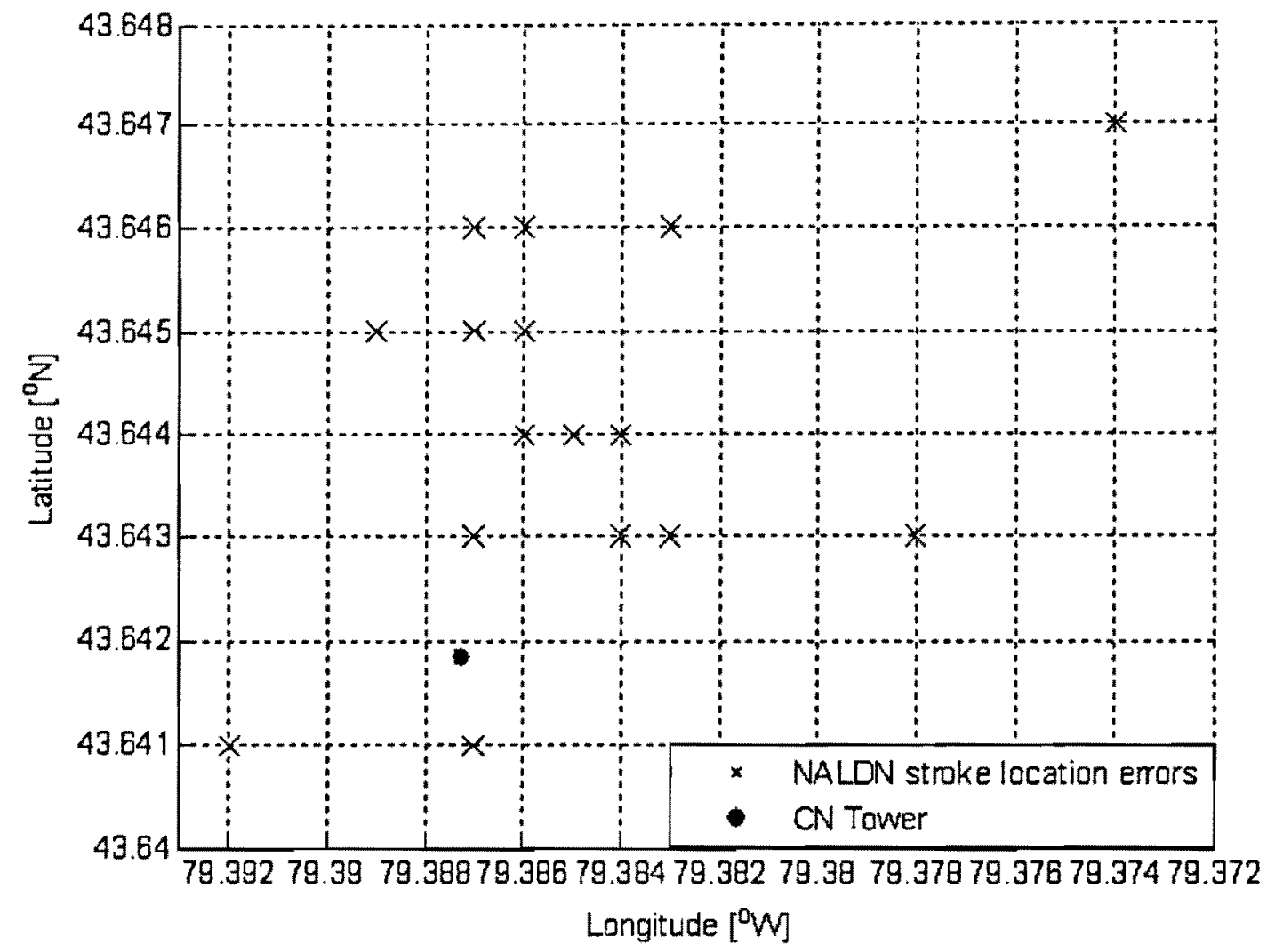

Figure 4.2.12: A close latitude verses longitude view of NALDN stroke location errors for the 22 detected strokes in 7 flashes. 
Figure 4.2.13 shows a North-South verses East-West distance plot of NALDN stroke location errors for 22 detected strokes in 7 flashes, with the origin corresponding to the location of the CN Tower. The horizontal and vertical axes correspond to the East-West (with East being positive) and North-South (with North being positive) error components, respectively.

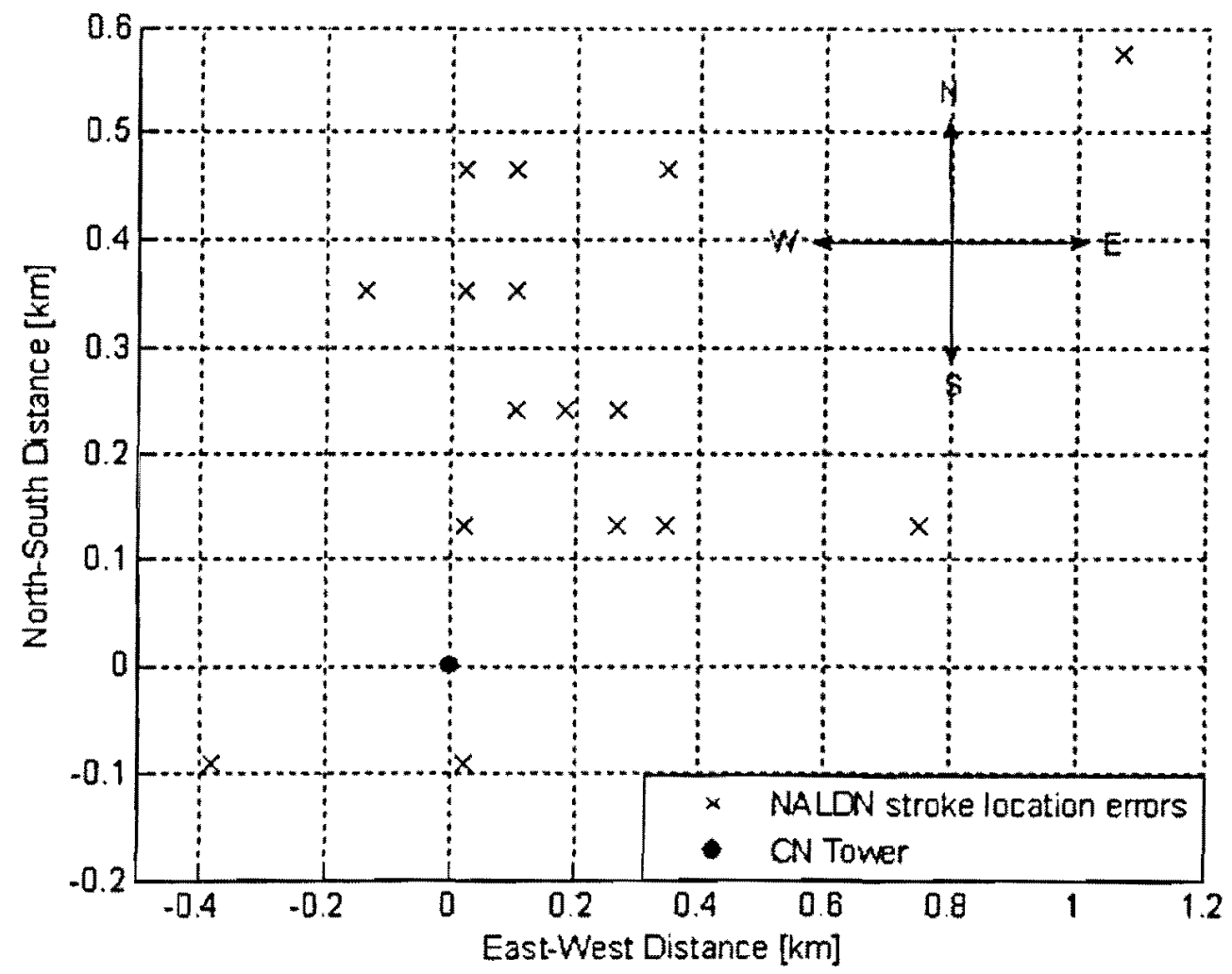

Figure 4.2.13: North-South verses East-West distance plot of NALDN stroke location errors in kilometers.

\begin{tabular}{|l|l|l|}
\hline & North-South $(\mathrm{km})$ & East-West $(\mathrm{km})$ \\
\hline Mean & 0.262 & 0.162 \\
\hline Median & 0.242 & 0.104 \\
\hline Standard Deviation & 0.174 & 0.292 \\
\hline
\end{tabular}

Table 4.2.5: The mean and median North-South and East-West NALDN location errors relative to the $\mathrm{CN}$ Tower, along with the corresponding standard deviations. 
Figure 4.2.14 shows a histogram of the NALDN absolute location errors for 22 detected strokes from 7 flashes. Each column corresponds to an absolute location error range with a bin size of $0.1 \mathrm{~km}$.

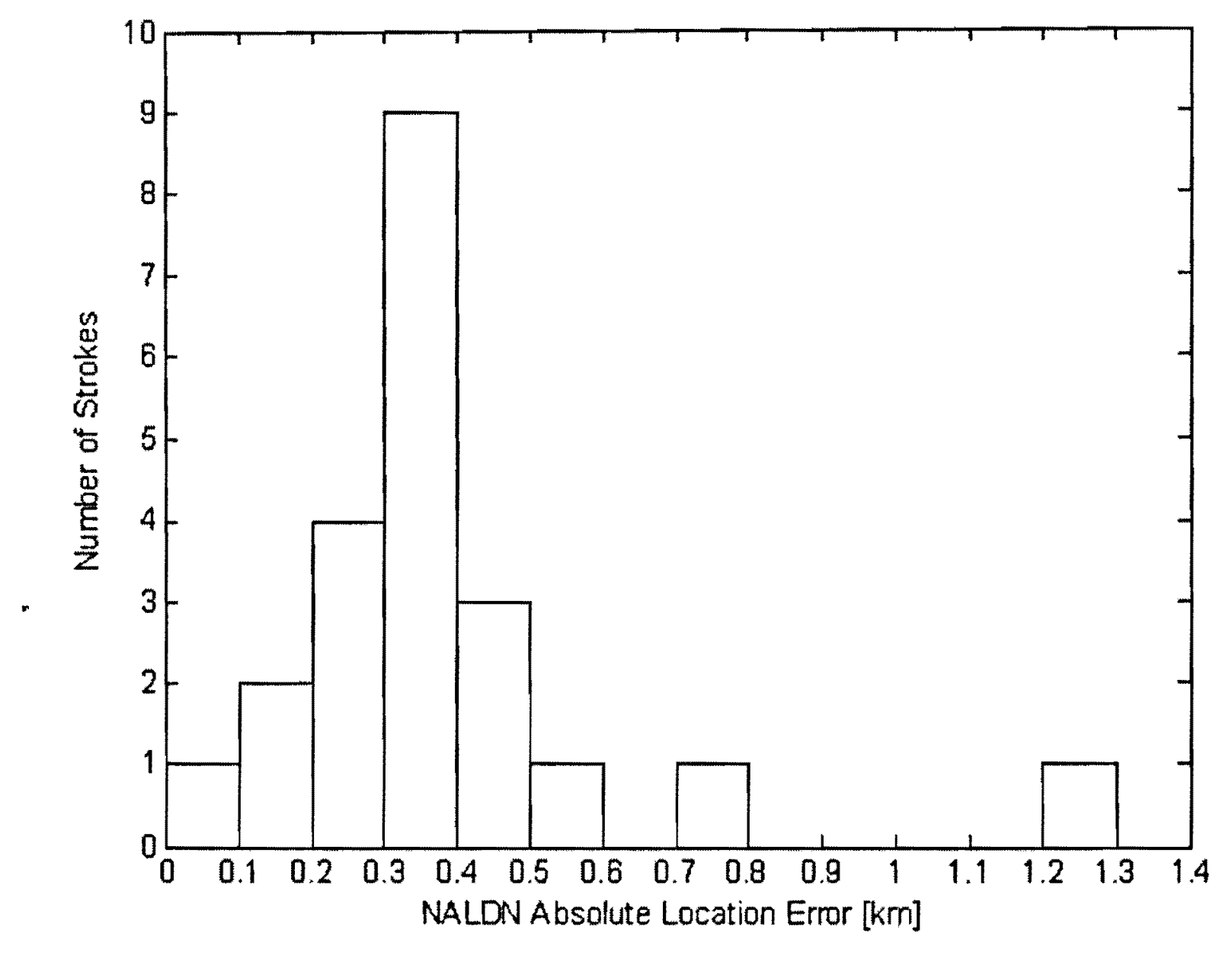

Figure 4.2.14: Histogram of the NALDN absolute location errors. 
Figure 4.2.15 shows the NALDN absolute location error plotted versus the CN Tower current wavefront maximum steepness for 22 detected strokes from 7 flashes.

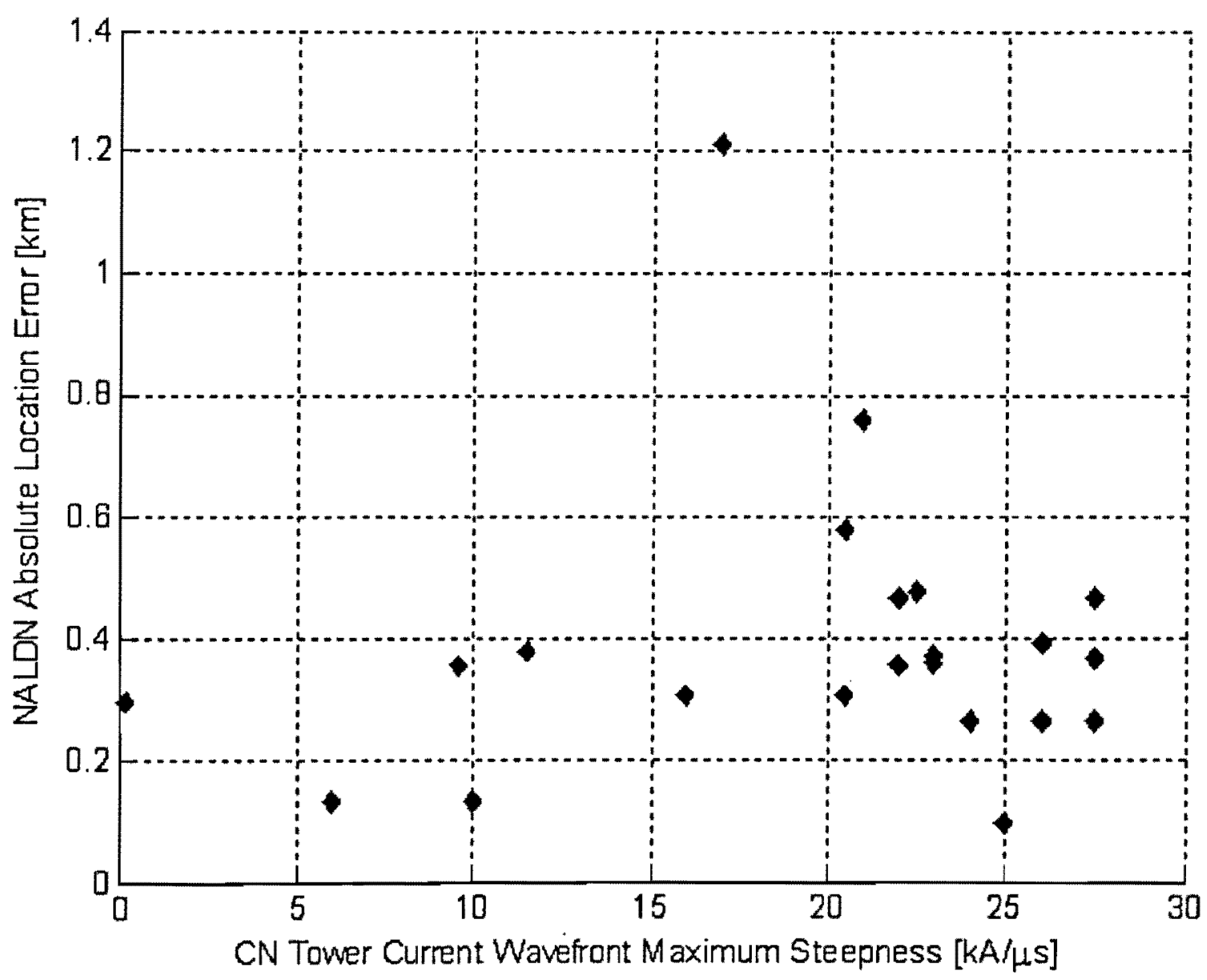

Figure 4.2.15: NALDN absolute location error plotted versus CN Tower current wavefront maximum steepness. 
Figure 4.2.16 shows the NALDN absolute location error plotted versus the CN Tower peak current for 22 detected strokes from 7 flashes.

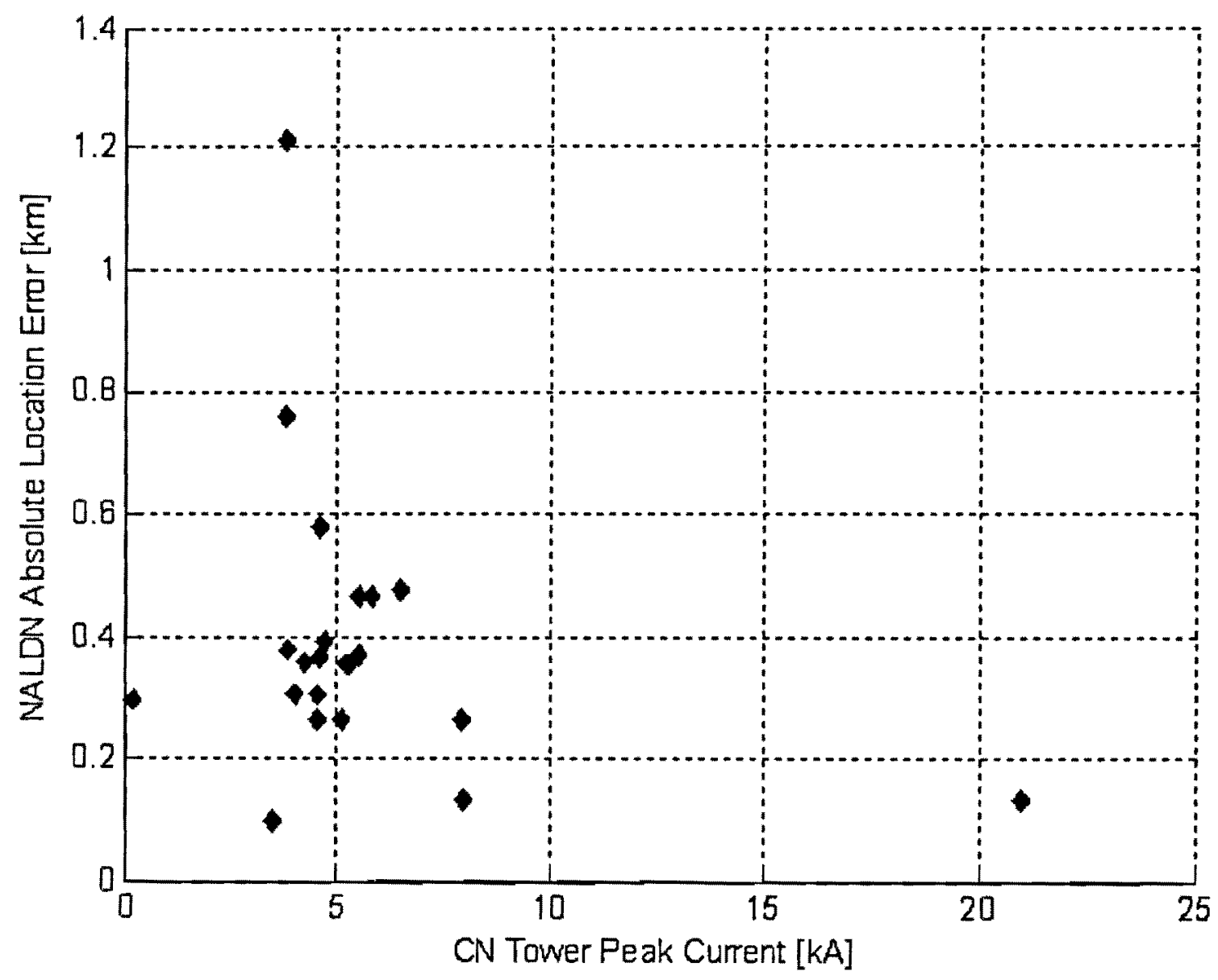

Figure 4.2.16: NALDN absolute location error plotted versus CN Tower peak current. 
Figure 4.2.17 shows the NALDN absolute location error plotted versus the peak of the azimuthal component of the magnetic field $\left(\mathrm{H}_{\phi}\right)$ measured $2 \mathrm{~km}$ north of the $\mathrm{CN}$ Tower. The total number of fields measured $2 \mathrm{~km}$ north of the CN Tower is 37 , out of which 21 were detected by the NALDN.

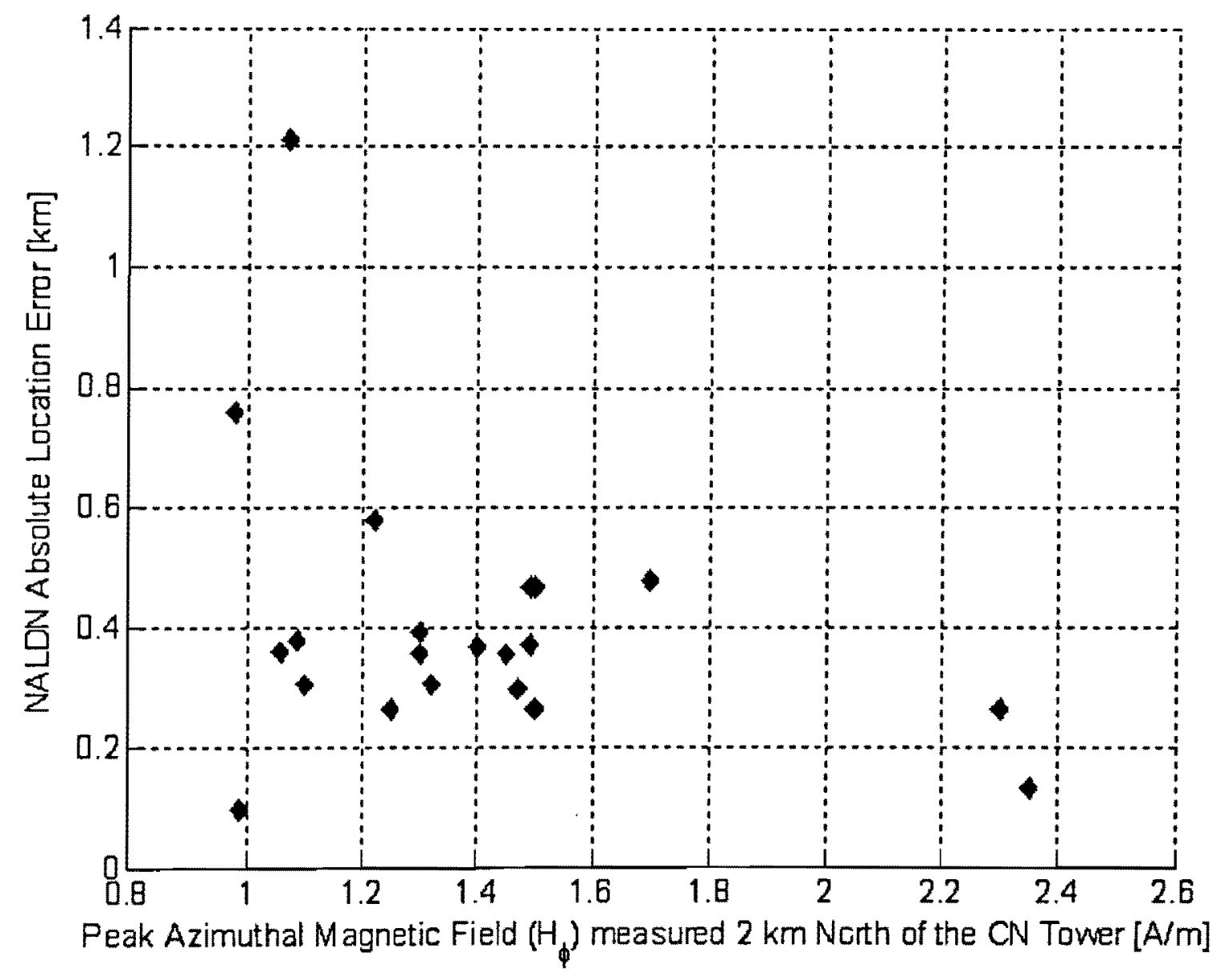

Figure 4.2.17: NALDN absolute location error plotted versus the peak of the azimuthal component of the magnetic field $\left(\mathrm{H}_{\phi}\right)$ measured $2 \mathrm{~km}$ north of the CN Tower. 
Figure 4.2.18 shows the NALDN absolute location error plotted versus the peak of the vertical component of the electric field $\left(\mathrm{E}_{z}\right)$ measured $2 \mathrm{~km}$ north of the $\mathrm{CN}$ Tower. The total number of fields measured $2 \mathrm{~km}$ north of the CN Tower is 37 , out of which 21 were detected by the NALDN.

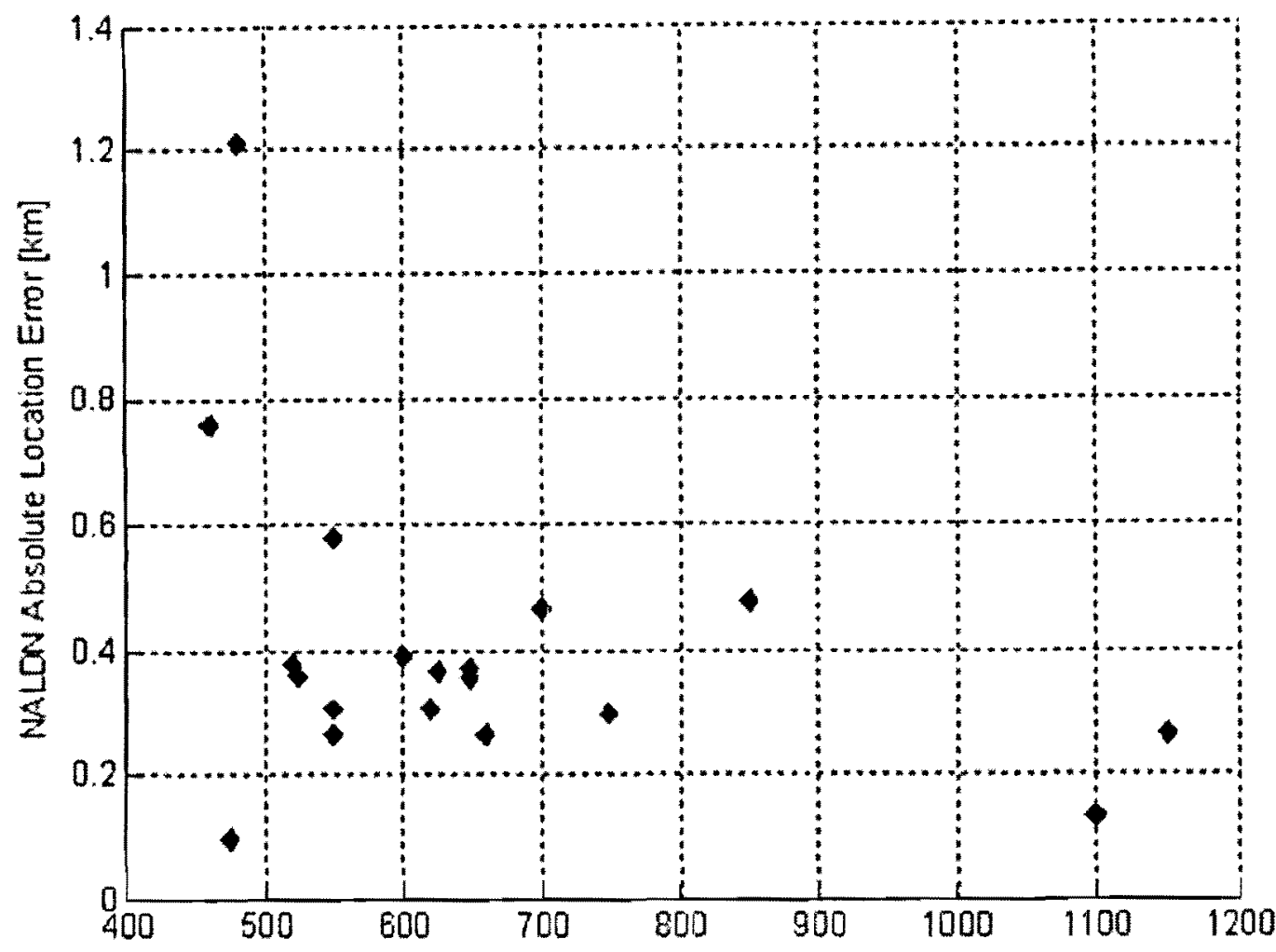

Peak Vertical Component of the Electric Field $\left(E_{2}\right)$ measured $2 \mathrm{~km}$ North of the $C N$ Tower $[V / m]$

Figure 4.2.18: NALDN absolute location error plotted versus the peak of the vertical component of the electric field $\left(\mathrm{E}_{z}\right)$ measured $2 \mathrm{~km}$ north of the CN Tower. 
The following figures from Figure 4.2.19 to Figure 4.2.24 provide statistics regarding the $50 \%, 90 \%$ and $99 \%$ error ellipses of each stroke. As described in Section 3.5.1, the error ellipse provides a region centered around the computed stroke location where there is a certain probability $(50 \%, 90 \%$ and $99 \%$ for the data provided) that the stroke occurred. Figure 4.2.19 shows the NALDN 50\% error ellipse semi-major axis length plotted versus the CN Tower peak current for 22 strokes in 7 flashes. Nearly all strokes, 21 out of 22 , have a semi-major axis length of $0.4 \mathrm{~km}$, with one stroke having an axis length of $0.7 \mathrm{~km}$.

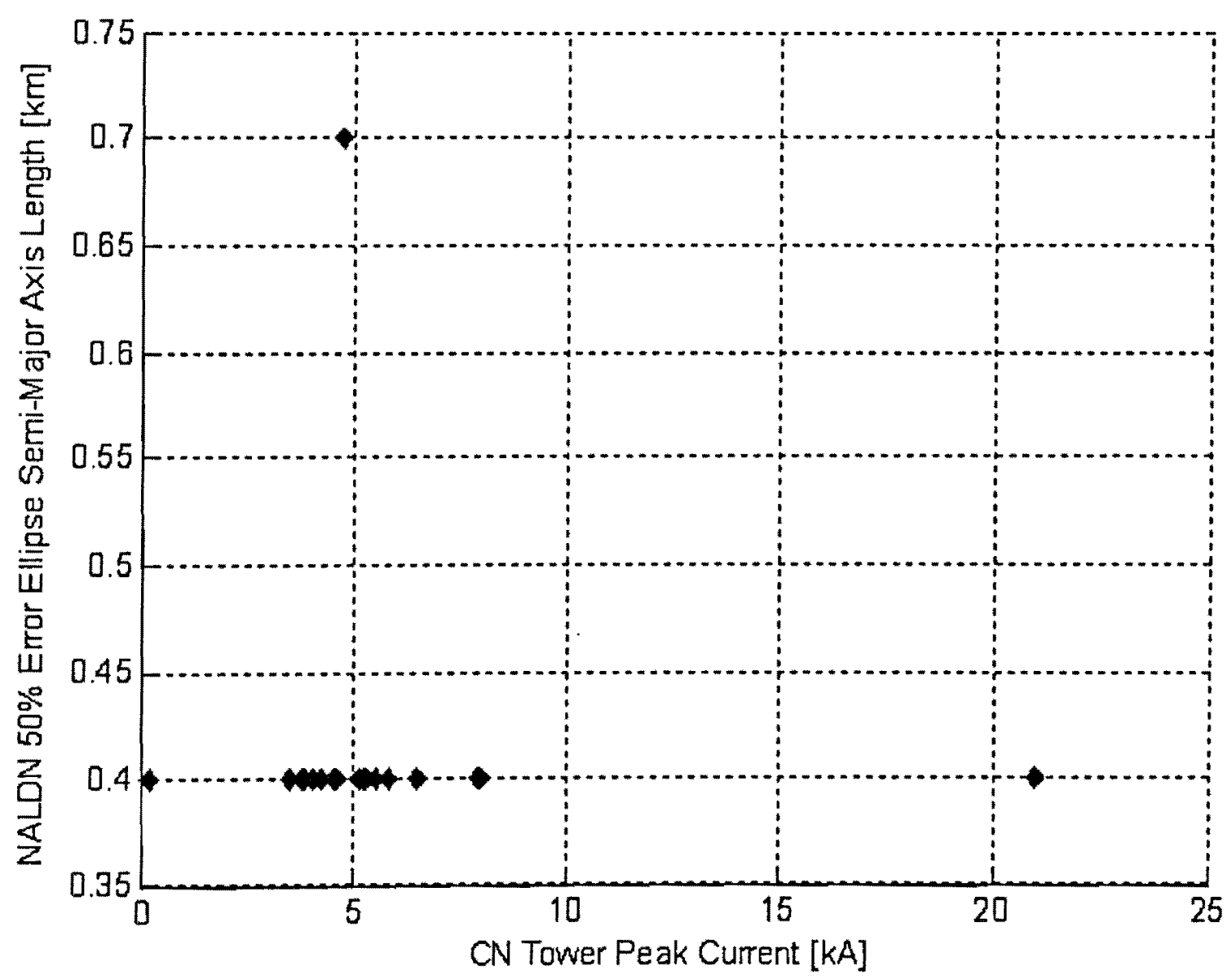

Figure 4.2.19: NALDN 50\% error ellipse semi-major axis length versus CN Tower peak current. 
Figure 4.2.20 shows the NALDN absolute location error plotted versus the NALDN $50 \%$ error ellipse semi-major axis length for 22 strokes in 7 flashes. The error ellipses for all events with an $0.4 \mathrm{~km}$ semi-major axis length (21 out of 22 strokes) are perfectly circular, meaning that the strokes located below the $50 \%, 90 \%$ and $99 \%$ lines have locations enclosed by the respective error ellipse. The one stroke that has a semi-major axis length of $0.7 \mathrm{~km}$, has a corresponding semi-minor axis length of $0.41 \mathrm{~km}$ and a location error of $0.39 \mathrm{~km}$.

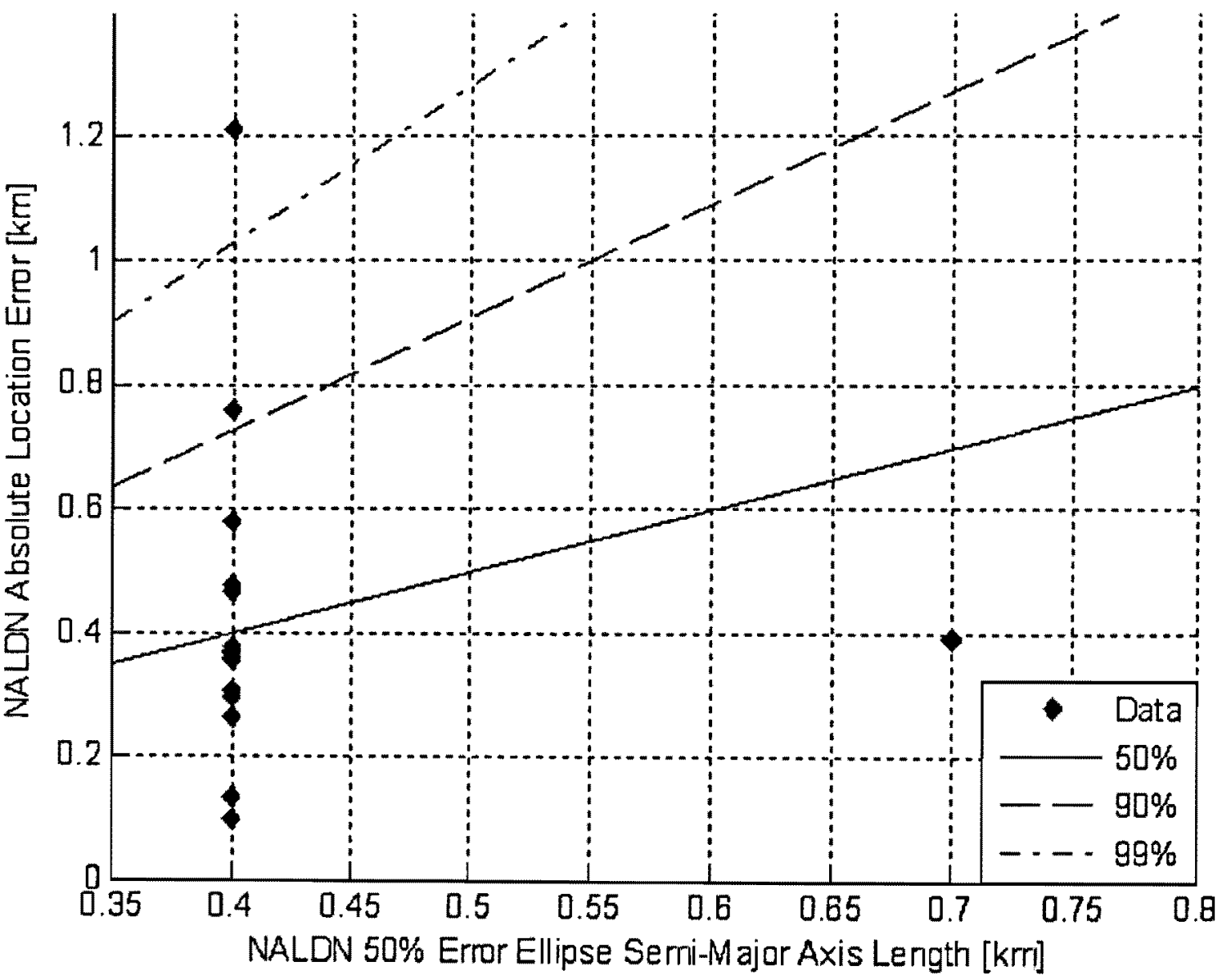

Figure 4.2.20: NALDN absolute location error plotted versus NALDN $50 \%$ error ellipse semi-major axis length. 
Figure 4.2.21 shows the NALDN 90\% error ellipse semi-major axis length plotted versus the CN Tower peak current for 22 strokes in 7 flashes. Nearly all strokes, 21 out of 22 , have a semi-major axis length of $0.73 \mathrm{~km}$, with one stroke having an axis length of $1.28 \mathrm{~km}$.

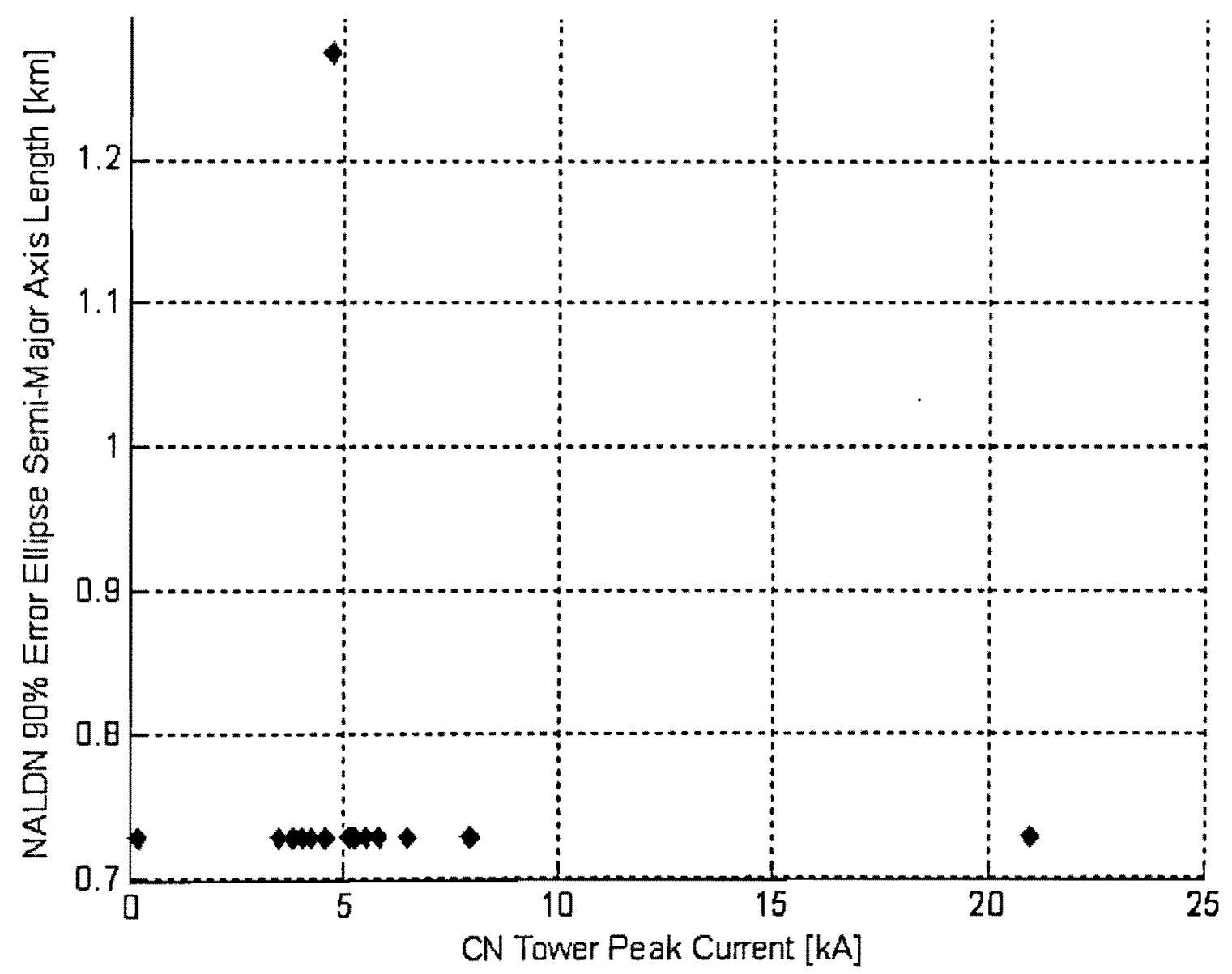

Figure 4.2.21: NALDN 90\% error ellipse semi-major axis length versus CN Tower peak current. 
Figure 4.2.22 shows the NALDN absolute location error plotted versus the NALDN $90 \%$ error ellipse semi-major axis length for 22 strokes in 7 flashes. The error ellipses for all events with an $0.73 \mathrm{~km}$ semi-major axis length (21 out of 22 strokes) are perfectly circular, meaning that the strokes located below the 50\%, $90 \%$ and $99 \%$ lines have locations enclosed by the respective error ellipse. The one stroke that has a semi-major axis length of $1.28 \mathrm{~km}$, has a corresponding semi-minor axis length of $0.75 \mathrm{~km}$ and a location error of $0.39 \mathrm{~km}$.

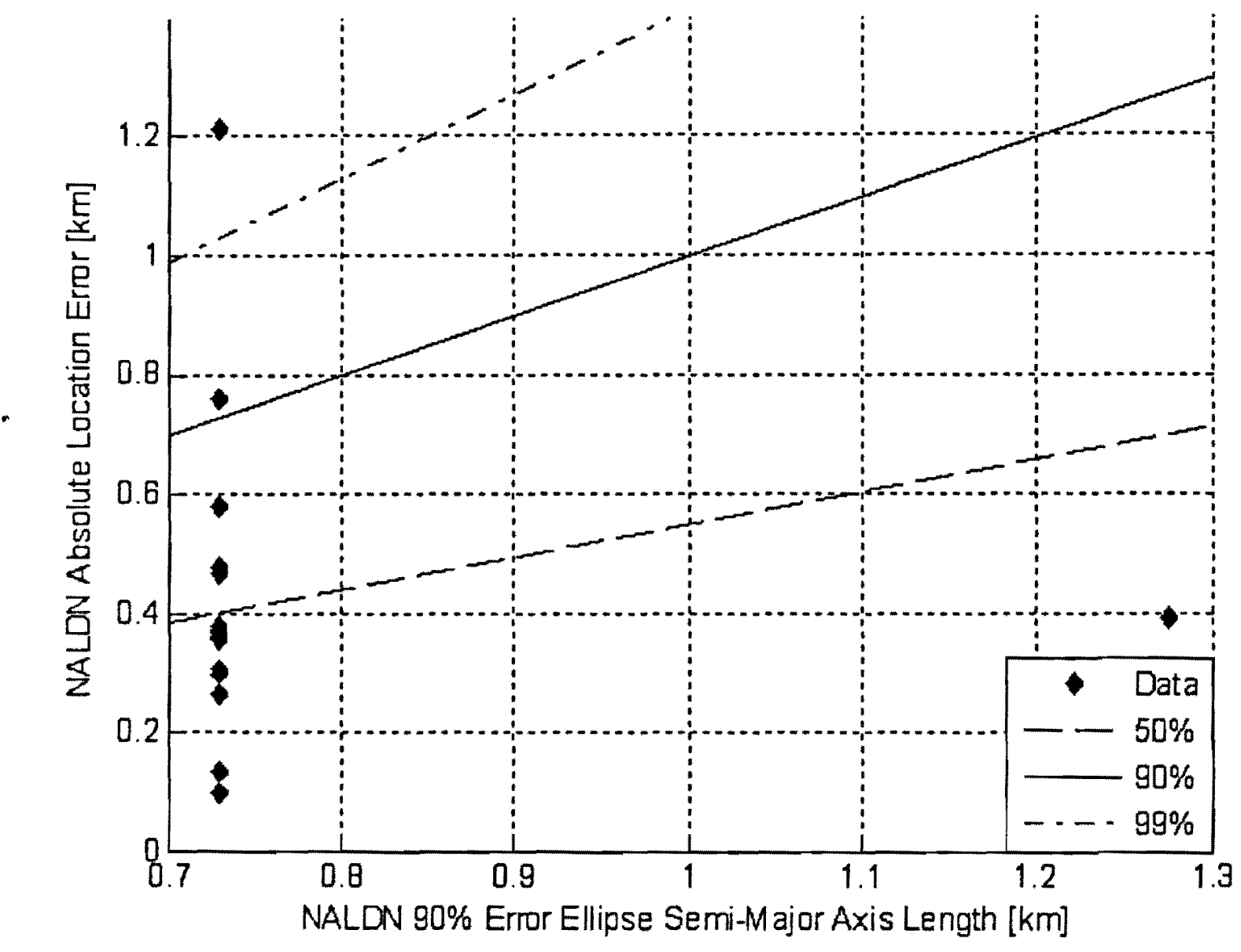

Figure 4.2.22: NALDN absolute location error plotted versus NALDN $90 \%$ error ellipse semi-major axis length. 
Figure 4.2.23 shows the NALDN 99\% error ellipse semi-major axis length plotted versus the CN Tower peak current for 22 strokes in 7 flashes. Nearly all strokes, 21 out of 22 , have a semi-major axis length of $1.03 \mathrm{~km}$, with one stroke having an axis length of $1.8 \mathrm{~km}$.

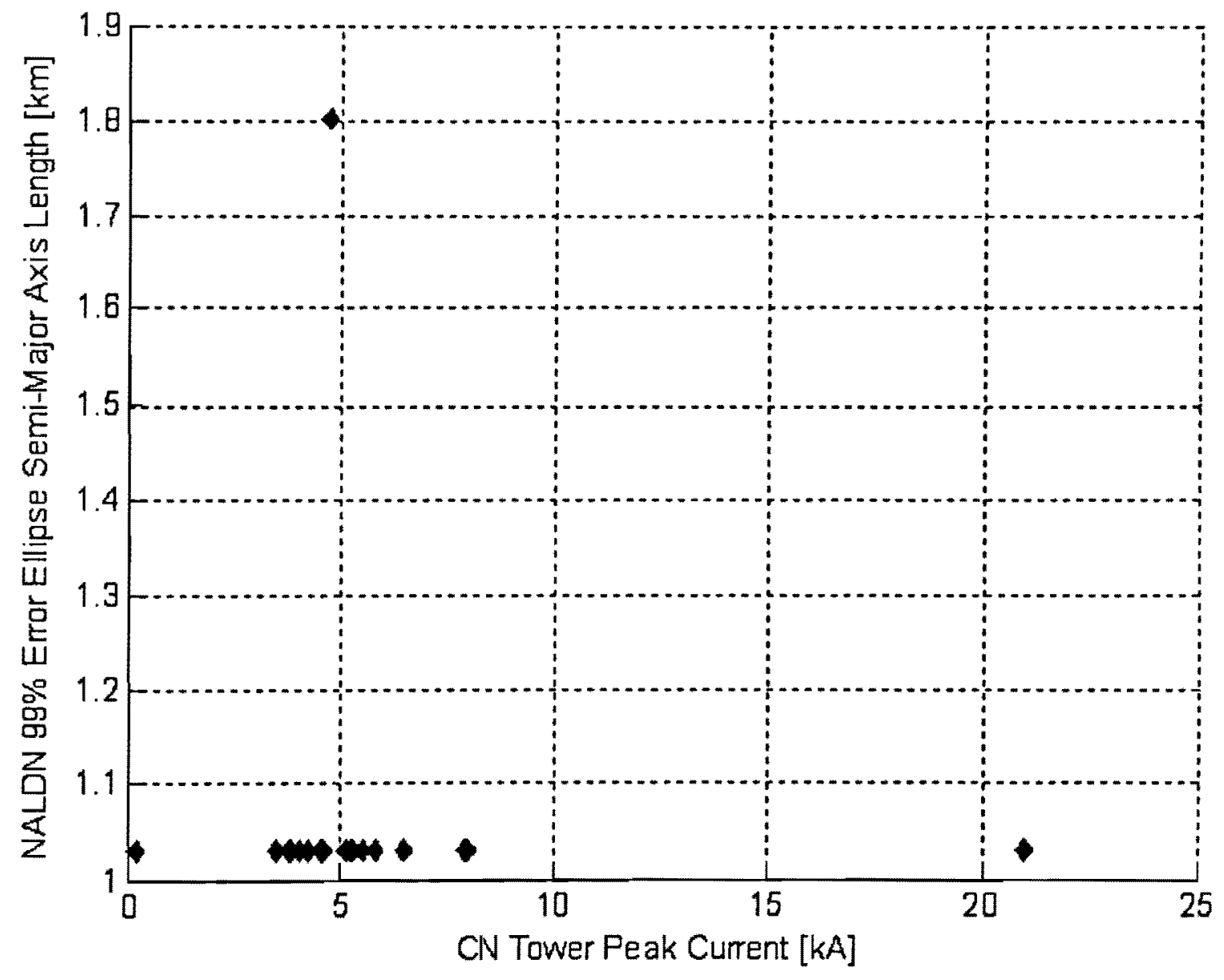

Figure 4.2.23: NALDN 99\% error ellipse semi-major axis length versus CN Tower peak current. 
Figure 4.2.24 shows the NALDN absolute location error plotted versus the NALDN $99 \%$ error ellipse semi-major axis length for 22 strokes in 7 flashes. The error ellipses for all events with an $1.03 \mathrm{~km}$ semi-major axis length (21 out of 22 strokes) are perfectly circular, meaning that the strokes located below the 50\%, 90\% and $99 \%$ lines have locations enclosed by the respective error ellipse. The one stroke that has a semi-major axis length of $1.8 \mathrm{~km}$, has a corresponding semi-minor axis length of $1.06 \mathrm{~km}$ and a location error of $0.39 \mathrm{~km}$.

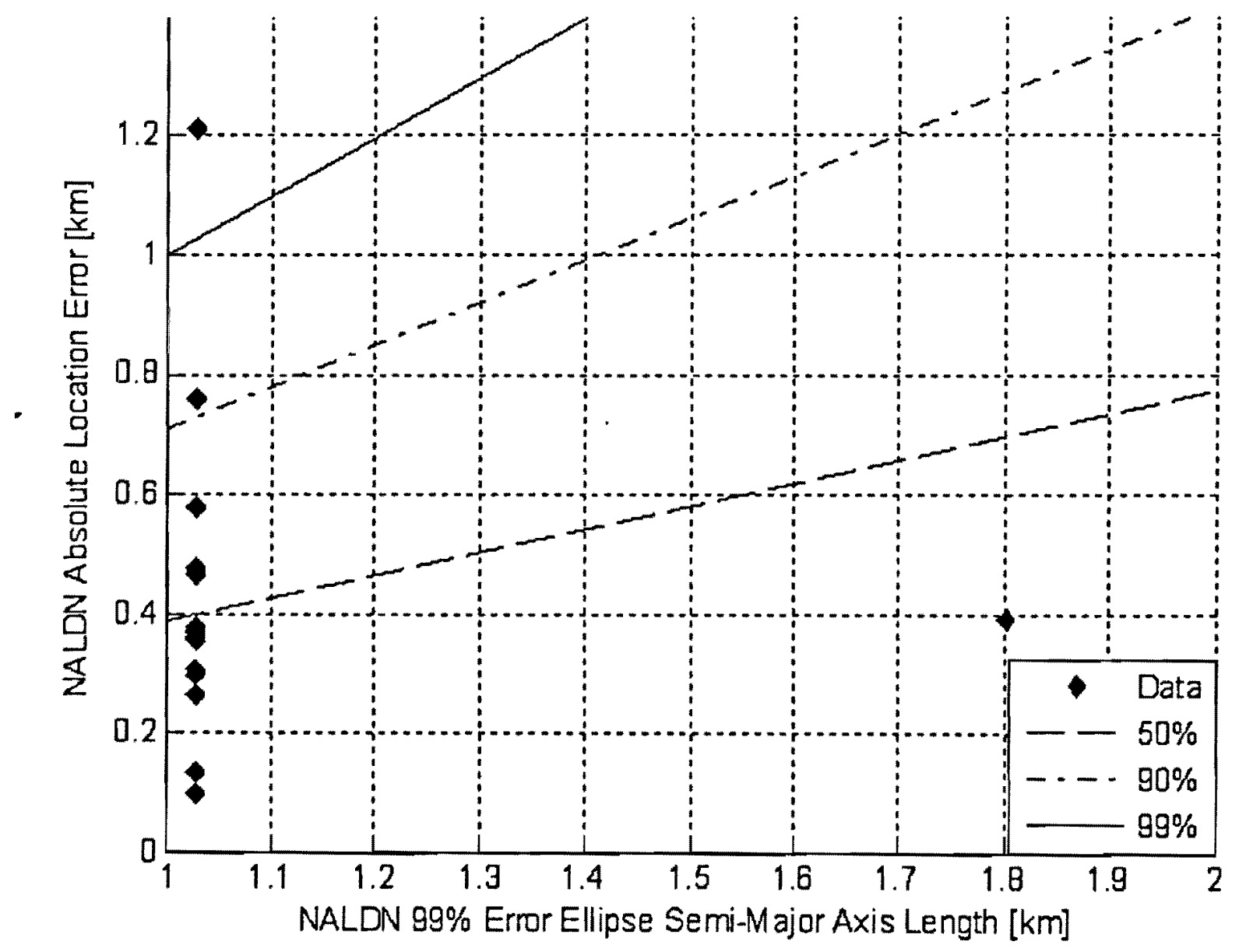

Figure 4.2.24: NALDN absolute location error plotted versus NALDN 99\% error ellipse semi-najor axis length. 


\subsubsection{Peak Current Estimation}

Figure 4.2.25 shows the NALDN estimated peak current plotted versus the CN Tower peak current for 22 strokes in 7 flashes. The best-fit linear regression equation: $I_{N A L D N}=$ $6.34 I_{C N T}-7.18$, is plotted in the figure. It should be noted that the two extraneous data points were omitted in the linear regression calculation.

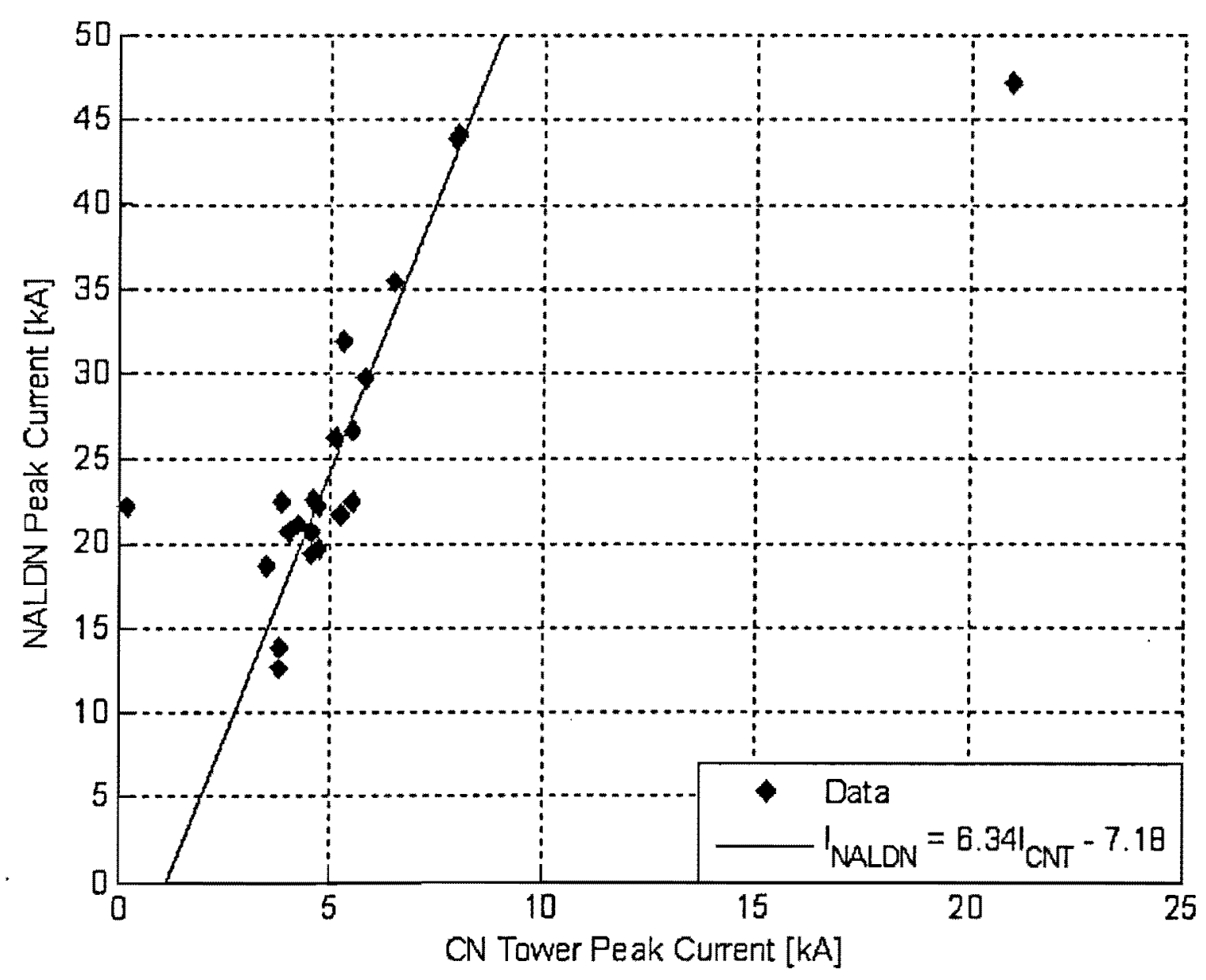

Figure 4.2.25: NALDN estimated peak current versus CN Tower peak current. 
Figure 4.2.26 shows the NALDN peak current estimation error, defined as $\triangle \mathrm{I}=\mathrm{I}_{N A L D N}$ - $\mathrm{I}_{C N T}$, plotted versus the CN Tower peak current for 22 strokes in 7 flashes. The best-fit linear regression equation: $\Delta I=5.34 I_{C N T}-7.18$, is also plotted in the figure. It should be noted that the two extraneous data points were omitted in the linear regression calculation.

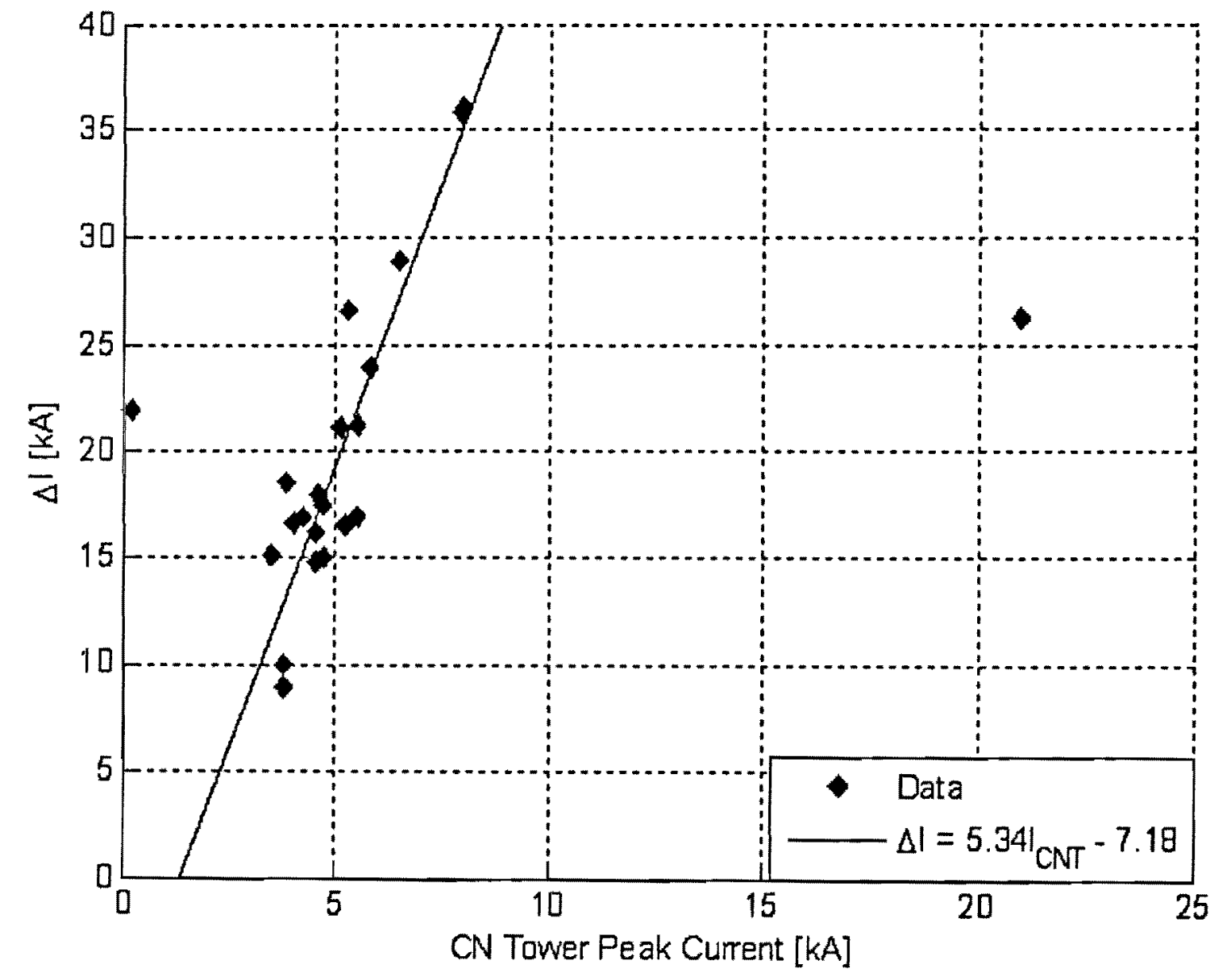

Figure 4.2.26: NALDN peak current estimation error $\left(\triangle \mathrm{I}=\mathrm{I}_{N A L D N}-\mathrm{I}_{C N T}\right)$ plotted versus CN Tower peak current. 


\subsection{Discussion}

\subsubsection{Detection Efficiency}

As shown in Table 4.2.2, the NALDN detected 7 out of the 9 flashes recorded at the CN Tower for the Toronto lightning season in 2005, resulting in an overall flash detection efficiency (DE) of $78 \%$. As shown in Table 4.2.1, the two flashes that went undetected (on August 19, 2005 at 11:57:14 (UTC) and 12:56:35 (UTC)) had extremely low peak currents. The stroke current waveforms did not resemble normal CN Tower lightning return strokes and the inter-stroke intervals were very small, between 1 to $2 \mathrm{~ms}$. These events are understood to be composed of lightning M-components, which are perturbations or transient impulses superimposed on a continuing current [50]. As shown in Table 4.2.2, because of the low signal level (peak currents from 100 to $200 \mathrm{~A}$ ) these events did not radiate electromagnetic field pulses of enough magnitude to trigger the field measurement system placed $2 \mathrm{~km}$ north of the CN Tower. So it is to be expected that the NALDN was unable to detect these events. It should be noted that in the rocket-triggered evaluation performed at Camp Blanding, care was taken to distinguish between M-components and normal return strokes, in order to only include natural cloud-to-ground lightning in the evaluation [8]. Therefore, excluding these two events that the NALDN is not intended to detect, the NALDN detected 7 out of 7 flashes giving a 100\% flash DE as shown in Table 4.2.3 [52]. This data corresponds well to the $90 \%$ projected flash DE claimed for the Toronto area in Figure 3.2.3 and Table 3.6.1, although a larger data set is required for an accurate evaluation.

As shown in Table 4.2.2, the NALDN detected 22 out of the 47 strokes or events recorded at the CN Tower for the Toronto lightning season in 2005, resulting in an overall stroke DE of $47 \%$. Excluding the events thought to be M-components, the NALDN detected 22 out of 39 strokes, for a stroke DE of 56\%, as shown in Table 4.2.3. This corresponds well to the claims made in Table 3.6.1, that the CLDN should have a stroke DE of 40 to $50 \%$. However, 
because the CN Tower is so close to the U.S. border, two out of the three closest sensors to the CN Tower are IMPACT-ESP sensors, as shown in Table 4.2.4 and Figure 4.2.6. This may account for the slightly higher $56 \%$ stroke DE.

In Figure 4.2.7 the NALDN stroke detection efficiency is plotted as a function of current wavefront maximum steepness measured at the CN Tower. Generally the trend should be that as current wavefront maximum steepness increases, stroke DE increases because the magnitude of the electromagnetic field radiated is greater. Because of the small data set for certain ranges, this trend is somewhat obscured, particularly in the case of the 5 to 10 $\mathrm{kA} / \mu \mathrm{s}$ range that has a $100 \% \mathrm{DE}$ for its small set of 2 out of 2 detected strokes. It should be noted once again that 8 out of the 11 strokes in the 0 to $5 \mathrm{kA} / \mu$ s range are thought to be M-components.

Figure 4.2.8 shows the NALDN stroke DE plotted as a function of peak current measured at the $\mathrm{CN}$ Tower. It is interesting to note that strokes with peak currents of $4 \mathrm{kA}$ and above have a $100 \%$ DE. Strokes with peak currents from 3 to $4 \mathrm{kA}$ suffered a sharp drop in DE to $40 \%$, while strokes with peak currents from 1 to $3 \mathrm{kA}$ had a $0 \%$ DE. Eight out of the 9 strokes or events with peak currents from 0 to $1 \mathrm{kA}$ are thought to be M-components, so it is expected that these events will not be detected. Clearly a larger stroke peak current will lead to the emission of a greater magnitude electromagnetic field, which in turn can be detected by NALDN sensors. From this figure, it seems as if the CN Tower peak current values may be underestimated. According to Vaisala, for low current events only nearby sensors will detect a stroke. Given that the signal will need to be detectable at about 300 $\mathrm{km}$ (the distance from the CN Tower that will include enough sensors to produce a reliable location), the NALDN should detect $6 \mathrm{kA}$ events about half the time [51]. As mentioned in Section 3.6.3, the rocket-triggered evaluation performed at Camp Blanding in 2002 shows a stroke DE of $0 \%(0$ strokes detected out of 3$)$ for peak currents under $5 \mathrm{kA}$, and a stroke DE of $20 \%$ for peak currents between 5 to $10 \mathrm{kA}$ ( 2 strokes detected out of 10) [8]. In $2003 \mathrm{a}$ 
stroke DE of $0 \%$ (0 strokes detected out of 6$)$ was obtained for peak currents under $5 \mathrm{kA}$, and a stroke DE of $28 \%$ was obtained for peak currents between 5 to $10 \mathrm{kA}$ (8 strokes detected out of 29). It is recommended that the coil sensitivity of the CN Tower be calibrated, in order to determine the accuracy of the measured peak currents. If the coil sensitivity differs from the current value, then the peak currents should be recalculated. If the peak current is underestimated, then the current wavefront maximum steepness will also be underestimated and will require recalculation with the new coil sensitivity value.

Figure 4.2.9 shows the NALDN stroke detection efficiency plotted as a function of the peak of the azimuthal component of the magnetic field $\left(\mathrm{H}_{\phi}\right)$ measured $2 \mathrm{~km}$ north of the $\mathrm{CN}$ Tower. The total number of azimuthal fields measured $2 \mathrm{~km}$ north of the CN Tower is 37 , out of which 21 corresponding strokes were detected by the NALDN. The 8 events that are suspected of being M-components were not detected by the field measurement system, and are therefore not part of this data set. The azimuthal magnetic field is correlated to stroke $D E$, because a larger peak value for $\mathrm{H}_{\phi}$ will result in a greater chance of the stroke being detected by a sensor. It should be noted that for the $\mathrm{CN}$ Tower, the resulting magnetic field from lightning strokes will be almost completely azimuthal because of the completely vertical lightning path that the CN Tower creates for the bottom $553 \mathrm{~m}$. The NLDN IMPACTESP sensors detect and measure the magnetic field with 2 crossed loop antennas for the determination of a direction vector which points towards the stroke, as discussed in Section 3.4.1. As shown in Figure 4.2.9, strokes which generated a peak azimuthal magnetic field over $1.2 \mathrm{~A} / \mathrm{m}$ had a $100 \%$ DE. Strokes that generated a peak azimuthal magnetic field in the 1.0 to $1.2 \mathrm{~A} / \mathrm{m}$ range suffered a drop in $\mathrm{DE}$ to $57 \%$, with 4 out of 7 strokes detected. Only 2 out of 8 strokes were detected in the 0.8 to $1.0 \mathrm{~A} / \mathrm{m}$ peak $H_{\phi}$ range, resulting in a $25 \%$ DE. Lastly, none of the 7 strokes which generated a peak azimuthal magnetic field in the 0.6 to $0.8 \mathrm{kA}$ range were detected. This figure clearly demonstrates that a stroke with a larger peak current, which in turn emits a larger magnetic field, is more likely to be detected. 
Figure 4.2.10 displays the NALDN stroke detection efficiency plotted as a function of the peak of the vertical component of the electric field $\left(\mathrm{E}_{z}\right)$ measured $2 \mathrm{~km}$ north of the $\mathrm{CN}$ Tower. As for $\mathrm{H}_{\phi}$, the total number fields measured $2 \mathrm{~km}$ north of the CN Tower is 37, out of which 21 corresponding strokes were detected by the NALDN. The M-component events were not detected by the field measurement system and are therefore not part of this data set. The vertical component of the electric field is correlated to stroke $\mathrm{DE}$, because a larger peak value for $\mathrm{E}_{\boldsymbol{z}}$ will result in a greater chance of the stroke being detected by a sensor. The CLDN LPATS-IV sensors will detect the electric field using a whip antenna and create a time-of-arrival (TOA) range circle, as discussed in Section 3.4.1. The NLDN IMPACT-ESP sensors will also use a plate antenna to detect the electric field and determine a TOA range circle, which is also mentioned in Section 3.4.1. As shown in Figure 4.2.10, strokes which generated a peak $\mathrm{E}_{z}$ field over $600 \mathrm{~V} / \mathrm{m}$ had a $100 \% \mathrm{DE}$. The DE then gradually falls to $75 \%$ ( 3 out of 4 strokes detected) in the 550 to $600 \mathrm{~V} / \mathrm{m}$ range, $67 \%$ ( 2 out of 3 strokes detected) in the 500 to $550 \mathrm{~V} / \mathrm{m}$ range and $50 \%$ ( 3 out of 6 strokes detected) in the the 450 to 500 $\mathrm{V} / \mathrm{m}$ range, before none of the 11 strokes with peak $\mathrm{E}_{z}$ fields below $450 \mathrm{~V} / \mathrm{m}$ are detected. Once again this demonstrates that a stroke with a larger peak current, which in turn emits a larger $\mathrm{E}_{z}$ field, is more likely to be detected.

\subsubsection{Location Accuracy}

Figures 4.2.11 to 4.2.13 display the NALDN stroke location errors for the 22 strokes in 7 flashes detected by the NALDN. In Figure 4.2.13, the origin corresponds to the location of the CN Tower. The horizontal and vertical axes correspond to the east-west (with east being positive) and north-south (with north being positive) error components, respectively. The NALDN stroke location error does seem to have a large bias towards the north of the CN Tower and a slight bias towards the east. This is shown in Table 4.2.5, where the mean 
stroke location error is shown to be $0.262 \mathrm{~km}$ north and $0.162 \mathrm{~km}$ east of the CN Tower. Similarly the median stroke location error is $0.242 \mathrm{~km}$ north and $0.104 \mathrm{~km}$ east of the CN Tower. Only one stroke was predicted as being south-west of the CN Tower, one stroke was predicted as being south-east of the CN Tower and one stroke was predicted as being north-west of the CN Tower. The remaining 19 strokes were predicted north-east of the $\mathrm{CN}$ Tower.

As shown in Table 4.2.2, for the 22 strokes detected by the NALDN, the median absolute location error relative to the CN Tower is $0.356 \mathrm{~km}$ and the mean error is $0.390 \mathrm{~km}$. This corresponds well to Vaisala's predicted $0.5 \mathrm{~km}$ median absolute location error in Figure 3.6.3 and Table 3.6.1. In Figure 4.2.14, which shows a histogram of the NALDN absolute location errors for the 22 strokes, it can be seen that the majority of the strokes ( 9 out of 22) had a location error between 0.3 to $0.4 \mathrm{~km}$. A possible reason for the very low median location error of $0.356 \mathrm{~km}$ obtained for CN Tower data, may be the fact that the CN Tower creates a perfectly vertical lightning path to ground for all strokes. For the NLDN IMPACTESP sensors, the magnetic field is sampled in the north-south and east-west loops at the initial peak of the return stroke magnetic field [10]. This peak is radiated from the bottom hundred meters of the lightning channel, during the first microseconds of the return stroke [1]. Because the bottom of a lightning channel tends to vertical, the magnetic fields will tend to be horizontal. In the case of a lightning strike to the $\mathrm{CN}$ Tower, the bottom $553 \mathrm{~m}$ of the lightning channel will always be completely vertical. This should reduce any magnetic direction finding (DF) angular errors caused by non-vertical lightning channels, which may well account for the low median location error of $0.356 \mathrm{~km}$. Although this is true, according to Vaisala, the main influence on location accuracy will come from the timing errors resulting from the differing propagation paths to the different sensors, which is unrelated to channel geometry [51].

Figure 4.2.15 shows the NALDN absolute location error plotted versus the CN Tower 
current wavefront maximum steepness for 22 detected strokes from 7 flashes. Interestingly it seems that as the stroke current wavefront maximum steepness increases, the absolute location error for the stroke also increases. This should not be the case because a greater maximum steepness should emit a greater electromagnetic field, allowing more sensors to detect the stroke and contribute to determining the stroke location. However, the data set is quite small and there are very few strokes with a current wavefront maximum steepness below $20 \mathrm{kA} / \mu \mathrm{s}$, so no decisive conclusions can be made.

Figure 4.2.16 shows the NALDN absolute location error plotted versus the CN Tower peak current for 22 detected strokes in 7 flashes. Although it's difficult to draw any decisive conclusions because of the small data set and the lack of strokes with peak currents in the 10 to $20 \mathrm{kA}$ range, it can generally been seen that as the return stroke peak current increases, the absolute location error decreases. This is because strokes with higher peak currents will emit an electromagnetic field of a greater magnitude and will therefore be detected by more sensors, allowing a better location estimate to be made. It should be mentioned once again that the $\mathrm{CN}$ Tower peak current values seem to be underestimated and that the coil sensitivity of the CN Tower should be checked with a calibration.

Figure 4.2.17 shows the NALDN absolute location error plotted versus the peak of the azimuthal component of the magnetic field $\left(\mathrm{H}_{\phi}\right)$ measured $2 \mathrm{~km}$ north of the $\mathrm{CN}$ Tower. The total number of fields measured is 37 , out of which 21 were detected by the NALDN. As expected, strokes with a higher peak azimuthal magnetic field generally obtained a lower absolute location error. This is because strokes with larger peak currents which in turn emit a larger $\mathrm{H}_{\phi}$ field, are more likely to be detected by more sensors, allowing these sensors to contribute to the location estimate of the stroke. The NLDN IMPACT-ESP sensors detect and measure the magnetic field with 2 crossed loop antennas for the determination of a direction vector which points towards the stroke, as discussed in Section 3.4.1.

Figure 4.2.18 shows the NALDN absolute location error plotted versus the peak of the 
vertical component of the electric field $(\mathrm{Ez})$ measured $2 \mathrm{~km}$ north of the $\mathrm{CN}$ Tower. Once again, the total number of fields measured 37 , out of which 21 were detected by the NALDN. As with the peak $\mathrm{H}_{\phi}$ field, strokes with a higher peak $\mathrm{E}_{z}$ field generally obtained a lower absolute location error. This is because a larger peak value of $E_{z}$ will result in the stroke being detected by more sensors, which will contribute to providing a better location estimate. The CLDN LPATS-IV sensors and the NLDN IMPACT-ESP sensors will detect the electric field and determine a TOA range circle for the stroke, as described in Section 3.4.1.

As described in Section 3.5.1, the 50\% error ellipse, calculated for each stroke location solution, is defined as a confidence region in which there is a $50 \%$ probability that the actual stroke location lies within the area circumscribed by the ellipse, with the center of the ellipse being the most probable stroke location [8]. Therefore, the $50 \%$ error ellipse indicates the median location accuracy of a stroke. The semi-major axes for the $50 \%$ error ellipse was provided with the NALDN data, while the $90 \%$ and $99 \%$ error ellipse semi-major axes were derived by multiplying the $50 \%$ semi-major axes by a scaling factor provided by Vaisala [51].

Figure 4.2.19 shows the NALDN 50\% error ellipse semi-major axis length plotted versus the $\mathrm{CN}$ Tower peak current for 22 detected strokes in 7 flashes. It is interesting to note that 21 out of the 22 strokes have a semi-major axis length of $0.4 \mathrm{~km}$, with a single stroke having a semi-major axis length of $0.7 \mathrm{~km}$. According to Vaisala, the reason for this is that the minimum value for the $50 \%$ error ellipse semi-major axis length is set at $0.4 \mathrm{~km}$, in order to account for propagation related bias errors that are not included in the error model [51]. Therefore, any ellipse semi-major or semi-minor axis less than $0.4 \mathrm{~km}$ (produced by the model) will be set to $0.4 \mathrm{~km}$ [51]. This tends to indicate that the median location accuracy for strokes to the $\mathrm{CN}$ Tower is $0.4 \mathrm{~km}$, or even less than this, since $0.4 \mathrm{~km}$ is the minimum value possible for the semi-major axis length. For the 22 strokes detected by the NALDN, the median absolute location error was indeed $0.356 \mathrm{~km}$. As previously mentioned, the reason for such a low median location error might be because the bottom $553 \mathrm{~m}$ of the lightning 
channel will always be completely vertical for strokes to the CN Tower. This should greatly reduce any magnetic direction finding (DF) angular errors caused by non-vertical lightning channels.

Figure 4.2.20 shows the NALDN absolute location error plotted versus the NALDN $50 \%$ error ellipse semi-major axis length, for the 22 detected strokes in 7 flashes. The $50 \%$ line contains the points for which the NALDN $50 \%$ semi-major axis length and absolute location error are equal, representing the boundary of the $50 \%$ error ellipse. The eccentricity (semimajor axis divided by the length of the semi-minor axis) is 1.0 for all events with an $0.4 \mathrm{~km}$ semi-major axis length (21 out of 22 strokes), making the ellipses for these events perfectly circular [52]. Because the error ellipses are circular, the data points below the $50 \%$ line correspond to strokes with locations enclosed by the $50 \%$ error ellipse. As displayed in Figure 4.2 .20 , it was found that $73 \%$ (16 out of the 22) detected strokes were enclosed by the $50 \%$ error ellipse. This includes the one stroke that has a semi-major axis length of 0.7 , with a corresponding semi-minor axis length of $0.41 \mathrm{~km}$ and a location error of $0.39 \mathrm{~km}$. A possible reason for having this very large percentage of $73 \%$ of the strokes enclosed by the $50 \%$ error ellipse may be due to the fact that the minimum value for the error ellipse axes is set at $0.4 \mathrm{~km}$ by Vaisala, as previously mentioned.

As previously mentioned, the $90 \%$ and $99 \%$ error ellipse data were derived by multiplying the $50 \%$ ellipse data by a scaling factor provided by Vaisala. This means that the same properties apply for this data as for the $50 \%$ data, meaning that the ellipses will be circular for 21 out of the 22 detected strokes. As shown in Figure 4.2.20, it was found that 91\% (20 out of the 22) detected strokes were enclosed by the $90 \%$ error ellipse and $95 \%$ (21 out of the 22) detected strokes were enclosed by the $99 \%$ error ellipse. Figures 4.2 .21 to 4.2 .24 display the same trends with the data scaled to the $90 \%$ and $99 \%$ error ellipse semi-major axis lengths. 


\subsubsection{Peak Current Estimation}

Figure 4.2.25 shows the NALDN estimated peak current versus the CN Tower peak current for 22 strokes in 7 flashes. There is a strong positive linear relationship between the CN Tower recorded and NALDN estimated peak currents, with the linear regression equation being: $I_{N A L D N}=6.34 I_{C N T}-7.18$. Figure 4.2 .26 shows the NALDN peak current estimation error $\left(\Delta I=I_{N A L D N}-I_{C N T}\right)$ plotted versus CN Tower peak current. The best-fit linear regression equation is: $\Delta I=5.34 I_{C N T}-7.18$. It should be noted that the two extraneous data points in the figures were omitted in the linear regression calculations. Because of uncertainties regarding a calibration constant of the CN Tower current derivative measurement system, no conclusive comments can be made regarding the peak current estimation of the NALDN until a calibration is performed.

However, it should be noted that the NALDN was developed to detect and estimate the peak current for strokes occurring from natural cloud-to-ground lightning, not the strokes from lightning to tall structures such as the $\mathrm{CN}$ Tower. The current waveforms obtained at the $\mathrm{CN}$ Tower are unlike natural cloud-to-ground current waveforms. The current waveforms contain several distinct peaks which correspond to reflections from the structural discontinuities of the CN Tower. The first peak in the current waveform which can be seen at the $10 \mu \mathrm{s}$ mark in Figure 4.2.3, corresponds to the initial peak from the lightning stroke. The largest absolute peak in the stroke current waveform corresponds to the reflection from ground. For cloud-to-ground lightning, this peak does not occur. However, the CN Tower peak current results are beneficial for determining trends which may be applicable to tall structures or objects in high altitude or mountainous areas. 


\subsubsection{Comparison to the Findings from other Evaluations}

Although a similar test was performed using rocket-triggered lightning in Florida at Camp Blanding in [8], this test evaluated a completely different region of the NALDN. The CN Tower is located on the border of the CLDN and the NLDN, surrounded by NLDN IMPACTESP sensors and CLDN LPATS-IV sensors, with two out of the three closest sensors to the CN Tower being IMPACT-ESP sensors. The rocket-triggered evaluation validated performance for a single location, north-east Florida, which is a particularly challenging region of the NLDN, since geographic characteristics limit the number of sensors that are close enough to the test site to detect strokes [5]. Furthermore, rocket-triggered lightning artificially initiates a lightning discharge, whereas lightning events to the CN Tower occur naturally and are similar to discharges that occur at tall structures or objects at high altitude or mountainous areas.

It should be noted that in the rocket-triggered evaluation performed over three years in [8], the sensors configuration varied greatly from year to year. In 2001, the sensors around Camp Blanding were not yet upgraded, having a combination of first generation IMPACT sensors and LPATS-IV sensors. In 2002, the NLDN upgrade began and Camp Blanding was surrounded by third generation IMPACT-ESP sensors and LPATS-IV sensors. In 2003, the NLDN upgrade was nearly completed with all sensors now being IMPACT-ESP sensors, except for one LPATS-IV sensor which was upgraded after the evaluation.

As mentioned in Section 3.6.2, in the rocket-triggered evaluation the NALDN was found to have a flash DE of $82 \%$ (9 out of 11 flashes detected) in $2001,86 \%$ (12 out of 14 flashes detected) in 2002, and $83 \%$ (10 out of 12 flashes detected) in 2003 [8]. In this evaluation, using CN Tower lightning data, the NALDN detected 7 out of 7 flashes (excluding M-components) for a $100 \%$ flash DE. Another evaluation of the 2003 upgrade of the NLDN was performed for the Tucson, Arizona area by the University of Arizona in [6]. During the summer of 2003, a 
digital video recording system with GPS time was used to record 671 cloud-to-ground flashes in 18 thunderstorms near Tucson, Arizona [6]. According to [6], the average flash DE of the NALDN near Tucson was approximately 95\%. During 2001, the same video system was used to record 109 flashes in 5 thunderstorms, with the NALDN obtaining a flash DE of $71 \%$ [7]. The improved flash DE in 2003 from the network upgrade is evident.

As mentioned in Section 3.6.3, in the rocket-triggered evaluation the NALDN was found to have a stroke DE of $52 \%$ (17 out of 33 strokes detected) in 2001, $57 \%$ ( 44 out of 77 strokes detected) in 2002 and $69 \%$ (34 out of 49 strokes detected) in 2003 [8]. In this evaluation, the NALDN detected 22 out of 39 strokes (excluding M-components), for a stroke DE of $56 \%$. This is similar to the 2002 results obtained in the rocket-triggered evaluation, when the Camp Blanding area was surrounded by a mix of LPATS-IV and IMPACT-ESP sensors, much like the CN Tower currently is. After the upgrade was nearly completed in 2003, the improved stroke DE is evident. From the Arizona evaluation performed in 2003, the 671 flashes contained at least 2290 separate return strokes, and the average stroke DE of the NALDN near Tucson was found to be approximately $78 \%$ [6]. During 2001, the same video system was used to record 109 flashes containing 401 strokes in 5 thunderstorms, resulting in a stroke DE of $41 \%$ [7]. The improved stroke DE in 2003 from the network upgrade is evident.

In the rocket-triggered evaluation, the NALDN was found to have a median location error of $0.27 \mathrm{~km}$ (for 17 strokes) in $2001,0.83 \mathrm{~km}$ (for 44 strokes) in 2002 and $0.45 \mathrm{~km}$ (for 34 strokes) in 2003 [8]. In this evaluation, for the 22 strokes detected by the NALDN, the median absolute location error relative to the $\mathrm{CN}$ Tower is $0.356 \mathrm{~km}$. Approximately $66 \%$ (63 out of 95 strokes) and $96 \%$ (91 out of 95 strokes) of stroke locations were enclosed by the $50 \%$ and $90 \%$ error ellipses respectively in the rocket-triggered evaluation [8]. In this evaluation, $73 \%$ (16 out of the 22) detected strokes were enclosed by the $50 \%$ error ellipse, $91 \%$ (20 out of the 22 ) detected strokes were enclosed by the $90 \%$ error ellipse and $95 \%(21$ 
out of the 22) detected strokes were enclosed by the $99 \%$ error ellipse.

Because of uncertainties regarding a calibration constant of the CN Tower current derivative measurement system, no comparisons involving peak currents can be made until a calibration is performed.

It should be noted that since 2005, Environment Canada has been continuing with an upgrade plan for the CLDN, developed with input from Vaisala, that will swap out older LPATS-IV sensors in favor of new IMPACT LS7000 sensors in order to improve network performance in ten prioritized areas [53]. Therefore, performing this evaluation before a network upgrade was of critical importance in order to establish a basis for the future evaluations of the upgraded network. This evaluation will allow the improvements of the network upgrade to be evaluated with respect to the current network.

\subsection{Summary}

In this chapter, the results of the NALDN evaluation in the Toronto area were presented. Initially the CN Tower lightning project was discussed, showing how lightning data from the CN Tower can be used to evaluate every parameter provided by the NALDN. The location of a stroke can be determined using the $\mathrm{CN}$ Tower current derivative measurement system. The time of the stroke can be determined using the GPS time synchronization system (accurate to $1 \mu \mathrm{s}$ ) which was recently installed at the $\mathrm{CN}$ Tower current derivative measurement system and at the field measurement system located $2 \mathrm{~km}$ north of the CN Tower. The polarity of the stroke can be determined using the current derivative measurement system and the electric field measurement system. The peak current of a stroke is also measured by the current derivative measurement system. The confidence ellipses can also be verified by knowing the exact location and time of a stroke.

For the NALDN performance evaluation, the NALDN provided stroke data that is precise 
and accurate to $1 \mathrm{~ms}$. The CN Tower stroke data was matched to the NALDN data with a perfect $1 \mathrm{~ms}$ accuracy for each stroke. The NALDN detected 7 out of the 9 flashes recorded at the CN Tower over the Toronto lightning season in 2005, resulting in an overall flash (DE) of $78 \%$. Excluding the two flashes thought to be composed of M-components, the NALDN detected 7 out of 7 flashes giving a 100\% percent flash DE. The NALDN detected 22 out of the 47 strokes or events recorded at the CN Tower, for an overall stroke DE of $47 \%$. Excluding the events thought to be M-components, the NALDN detected 22 out of 39 strokes, resulting in a stroke DE of $56 \%$. Relative to the CN Tower, the NALDN was found to have a median absolute location error of $0.356 \mathrm{~km}$ for the 22 strokes it detected. It was also demonstrated that the NALDN stroke location error seems to have a large bias towards the north of the CN Tower and a slight bias towards the east, with 19 out of the 22 strokes predicted north-east of the CN Tower. The $50 \%, 90 \%$ and $99 \%$ error ellipses provided by the NALDN were also evaluated. It was found that $73 \%$ (16 out of the 22) detected strokes were enclosed by the $50 \%$ error ellipse, $91 \%$ (20 out of the 22 ) detected strokes were enclosed by the $90 \%$ error ellipse and $95 \%$ (21 out of the 22 ) detected strokes were enclosed by the $99 \%$ error ellipse. The minimum value for the $50 \%$ error ellipse axes is set at $0.4 \mathrm{~km}$ by Vaisala, and 21 out of the 22 detected strokes had a semi-major axis length of $0.4 \mathrm{~km}$, suggesting that the median location error for CN Tower strokes is $0.4 \mathrm{~km}$ or less. The $0.356 \mathrm{~km}$ median location error obtained for the 22 detected strokes appears to support this. An in-depth discussion of these results was given in this chapter, followed by a comparison to the findings from other evaluations. 


\section{Chapter 5}

\section{Conclusions and Recommendations}

\subsection{Conclusions}

Using CN Tower data acquired over the Toronto lightning season in 2005, the perfor- mance characteristics of the North American Lightning Detection Network (NALDN) were thoroughly evaluated. This evaluation was made possible by the installation of a GPS timing system accurate to $1 \mu$ s on the $\mathrm{CN}$ Tower current derivative measurement system, which allowed the CN Tower lightning strokes to be accurately matched to the strokes detected by the NALDN. Furthermore, the installation of GPS timing on the field measurement system, located $2 \mathrm{~km}$ north of the CN Tower, facilitated an evaluation of the NALDN data based on the CN Tower lightning generated fields.

Although a similar test was performed using rocket-triggered lightning in Florida at Camp Blanding, this test only evaluated the north-east Florida region, which is a particularly challenging region of the NLDN, since geographic characteristics limit the number of sensors that are close enough to the test site to detect strokes. Additionally, rocket-triggered lightning artificially initiates a lightning discharge, whereas lightning events to the CN Tower occur naturally and are similar to discharges that occur to tall structures or objects in high 
altitude or mountainous areas.

Since 2005, Environment Canada has been continuing with an upgrade plan developed with input from Vaisala that will swap out older LPATS-IV sensors in favor of new IMPACT LS7000 sensors, in order to improve network performance in ten prioritized areas. Performing this evaluation before a network upgrade was of critical importance in order to establish a basis for future evaluations of the upgraded network. This evaluation will allow the improvements of the network upgrade to be evaluated with respect to the current network.

In this evaluation, the NALDN detected 7 out of the 9 flashes recorded at the CN Tower over the Toronto lightning season in 2005, resulting in an overall flash (DE) of $78 \%$. Excluding the two flashes thought to be composed of M-components, the NALDN detected 7 out of 7 flashes giving a $100 \%$ percent flash DE. This data corresponds well to the $90 \%$ projected flash DE claimed for the Toronto area, although a larger data set is required to accurately evaluate the claimed flash $\mathrm{DE}$.

The NALDN detected 22 out of the 47 strokes or events recorded at the CN Tower, for an overall stroke DE of $47 \%$. Excluding the events thought to be M-components, the NALDN detected 22 out of 39 strokes, resulting in a stroke DE of $56 \%$. This corresponds well to Vaisala's claims that the CLDN should have a stroke DE from 40 to $50 \%$. However, because the $\mathrm{CN}$ Tower is so close to the U.S. border, two out of the three closest sensors to the CN Tower are IMPACT-ESP sensors, which may explain the higher stroke DE.

The NALDN stroke DE is clearly dependent on the peak current of the stroke. The NALDN had a $100 \%$ stroke DE for the strokes that had a CN Tower measured peak current above $4 \mathrm{kA}$. Clearly a larger stroke peak current will lead to the emission of a greater magnitude electromagnetic field, which in turn can be detected by NALDN sensors. Unfortunately it seems as if the CN Tower peak current values may be underestimated, because Vaisala claims that the NALDN should have an approximate $50 \% \mathrm{DE}$ for $6 \mathrm{kA}$ events [51]. It is rec- 
ommended that the coil sensitivity of the CN Tower be calibrated, in order to determine the accuracy of the measured peak currents. If the coil sensitivity differs from the present value, then the peak currents and the current wavefront maximum steepness must be recalculated.

The NALDN stroke DE is also clearly dependent on the peak magnetic field, since a larger peak value for the magnetic field will result in a greater chance of the stroke being detected by a sensor. Based on the CN Tower lightning generated fields, it was clearly demonstrated that as the peak of the return stroke magnetic field increases, the NALDN stroke DE increases. Similarly it was shown that the peak of the vertical component of the electric field $\left(\mathrm{E}_{z}\right)$, measured $2 \mathrm{~km}$ north of the CN Tower, is correlated to stroke DE, because strokes with larger peak values for $\mathrm{E}_{z}$ were detected more readily by the NALDN. Strokes with larger peak currents will emit larger magnetic and electric fields, resulting in a greater chance of the stroke being detected by an NALDN sensor.

Using CN Tower lightning data the location accuracy of the NALDN was also evaluated.

- Relative to the CN Tower, the NALDN was found to have a median absolute location error of $0.356 \mathrm{~km}$ and a mean error of $0.390 \mathrm{~km}$ for the 22 strokes it detected. This accuracy far exceeds Vaisala's predicted $0.5 \mathrm{~km}$ median location accuracy. A possible reason for this may be the fact that the $\mathrm{CN}$ Tower simulates a perfectly vertical lightning path to ground for all strokes, greatly reducing any magnetic direction finding (DF) angular errors caused by non-vertical lightning channels.

It was also demonstrated that the NALDN stroke location error seems to have a large bias towards the north of the $\mathrm{CN}$ Tower and a slight bias towards the east, with the mean stroke location errors being $0.262 \mathrm{~km}$ north and $0.162 \mathrm{~km}$ east of the CN Tower for the 22 detected strokes. Out of the 22 strokes, 19 were predicted north-east of the CN Tower.

It was also shown that as the peak current, peak azimuthal magnetic field, and the peak vertical component of the electric field increase, the predicted stroke absolute location error decreases. This is because strokes with higher peak currents will emit an electromagnetic 
field of a greater magnitude and will therefore be detected by more sensors, allowing a better location estimate to be made.

The 50\%, 90\% and 99\% error ellipses provided by the NALDN were also evaluated. It was found that $73 \%$ (16 out of the 22) detected strokes were enclosed by the $50 \%$ error ellipse, $91 \%$ (20 out of the 22 ) detected strokes were enclosed by the $90 \%$ error ellipse and $95 \%$ (21 out of the 22) detected strokes were enclosed by the $99 \%$ error ellipse. A possible reason for having a very large percentage of $73 \%$ of the strokes enclosed by the $50 \%$ error ellipse may be due to the fact that the minimum value for the error ellipse axes is set at 0.4 $\mathrm{km}$ by Vaisala, and 21 out of the 22 detected strokes had a semi-major axis length of 0.4 $\mathrm{km}$. This also suggests that the median location error for CN Tower strokes is $0.4 \mathrm{~km}$ or less, which was verified by the $0.356 \mathrm{~km}$ median location error obtained for the 22 detected strokes.

As previously mentioned, because of uncertainties regarding a calibration constant of the CN Tower current derivative measurement system, no conclusive comments can be made regarding the peak current estimation of the NALDN until a calibration is performed.

Therefore, the use of CN Tower lightning data allowed for an extensive evaluation of the NALDN performance characteristics. The flash DE, stroke DE, absolute location error, peak current estimation and location accuracy model $(50 \%, 90 \%$ and $99 \%$ error ellipses) were all evaluated.

\subsection{Recommendations}

Firstly, it is recommended that the coil sensitivity of the CN Tower be calibrated, in order to determine the accuracy of the measured peak currents. If the coil sensitivity differs from the present value, then the peak current and wavefront steepness for the 2005 lightning data must be recalculated. 
A high resolution High-Speed camera with GPS timing would be a useful instrument to acquire and install $2 \mathrm{~km}$ north of the $\mathrm{CN}$ Tower. This would allow the visual parameters of individual strokes to be matched to CN Tower and NALDN stroke data. This would also allow upward verses downward initiated flashes to be distinguished. Since the NALDN's main purpose is to detect natural downward cloud-to-ground lightning, it would be interesting to see how upward verses downward initiated flashes have an effect on NALDN performance.

In order to properly evaluate if the trajectory of a stroke has an effect on NALDN performance, it is recommended that another digital video recording system be installed directly to the west or east of the CN Tower. Presently the east-west trajectory of a lightning event can be observed using the digital video recording made $2 \mathrm{~km}$ north of the CN Tower, but it is also necessary to see the image of the north-south trajectory. Of course this proposed digital video recording system must have GPS timing.

In a future evaluations, it would also be useful to examine the relationship between - location accuracy and the number of sensors that detect a particular stroke. This information was not available at the time of this evaluation, but it is expected that the location accuracy is dependent on the number of detecting sensors, with the accuracy improving as more sensors detect a stroke. Similarly, it would be interesting to evaluate the peak current estimation accuracy with respect to the number of sensors that detect a stroke.

Henceforth, NALDN data should be collected alongside CN Tower lightning data for any flashes that strike the CN Tower. Since the CN Tower offers the rare opportunity of evaluating all NALDN parameters including flash DE, stroke DE, absolute location error, peak current estimation and the location accuracy model (50\%, 90\% and 99\% error ellipses) error, it is important that additional data be collected, so that more conclusive statistics and trends could be determined. Since 2005, Environment Canada has been continuing with an upgrade plan developed with input from Vaisala that will swap out older LPATS-IV sensors in favour of new IMPACT LS7000 sensors, in order to improve network performance in ten 
prioritized areas. Therefore, a new evaluation will be necessary in order to determine the effects of this network upgrade. 


\section{Bibliography}

[1] V.A. Rakov and M.A. Uman, "Lightning: Physics and Effects," Cambridge University Press, August 2003.

[2] Environment Canada, "The Canadian Lightning Detection Network," December 2002. http://www.weatheroffice.ec.gc.ca/lightning/index_e.html

[3] Environment Canada, "Minister Stewart announces Canadian lightning detection network," October 1997. [News Release] http://www.ec.gc.ca/press/ldn_n_e.htm

[4] Vaisala, "Vaisala's NLDN: U.S. National Lightning Detection Network," January 2004. [Online Brochure] http://www.lightningstorm.com/jsp/discover/nldn/NLDN_Brochure_B210412EN-

- A.Jan2004.pdf

[5] J.A. Cramer, K. L. Cummins, A. Morris, R. Smith and T.R. Turner, "Recent Upgrades to the U.S. National Lightning Detection Network," 18th International Lightning Detection Conference, Vaisala Inc., Tucson, Arizona, June 7-9, 2004.

[6] K.E. Kehoe and E.P. Krider, "NLDN Performance in Arizona," 18th Intemational Lightning Detection Conference, Vaisala Inc., Tucson, Arizona, June 7-9, 2004.

[7] N.G. Parker and E.P. Krider, "A Portable PC-based system for making optical and electromagnetic measurements of lightning," Joumal of Applied Meteorology, vol. 42, no. 6 , pp. 739-751, June 2003.

[8] J. Jerauld, V.A. Rakov, M.A. Uman, K.J. Rambo and D.M. Jordan, "An Evaluation of the Performance Characteristics of the NLDN using Triggered Lightning," 18th International Lightning Detection Conference, Vaisala Inc., Tucson, Arizona, June 7-9, 2004 .

[9] A.M. Hussein, "CN Tower Lightning Parameters: A Review," 2003 International Workshop on EM Radiation from Lightning to Tall Structures, Bologna, Italy, June $27,2003$. 
[10] K.L. Cummins and M.J Murphy, "Overview of lightning detection in the VLF, LF, and VHF frequency ranges," 16th International Lightning Detection Conference, Global Atmospherics, Inc., Tucson, Arizona, November 7-8, 2000.

[11] U. Finke and O. Kreyer, "Detect and Locate Lightning Events from Geostationary Satellite Observations - Report Part I: Review of existing lightning location systems," Institue für Meteorologie und Klimatologie, Universität Hannover, September 2002.

[12] D.E. Proctor, "A Hyperbolic System for Obtaining VHF Radio Pictures of Lightning," Journal of Geophysical Research, vol. 76, no. 6, pp. 1478-1489, February 1971.

[13] N. Herodotou, "Study of Peak Currents due to Lightning in Ontario using an LLP system," M.A.Sc. Thesis, University of Toronto, Toronto, Ontario, Canada, 1990.

[14] D.R. MacGorman and W.D. Rust, "The Electrical Nature of Storms," Oxford University Press, March 1998.

[15] E.P. Krider, R.C. Noggle and M.A. Uman, "A Gated, Wideband Magnetic Direction Finder for Lightning Return Strokes," Journal of Applied Meteorology, vol. 15, no. 3, pp. 301-306, March 1976.

[16] E.A. Lewis, R.B. Harvey, and J.E. Rasmussen, "Hyperbolic Direction Finding with Sferics of Transatlantic Origin," Journal of Geophysical Research, vol. 65, no. 7, pp. 1879-1905, July 1960.

[17] Sovereign Publications Limited, "Global Atmospherics, Inc.," May 2004. [News Release] http://www.sovereign-publications.com/global.htm

[18] K.L. Cummins, M.J. Murphy, E.A. Bardo, W.L. Hiscox, R.B. Pyle, and A.E. Pifer, "A Combined TOA/MDF Technology Upgrade of the U.S. National Lightning Detection Network," Journal of Geophysical Research, vol. 103, no. D8, pp. 9035-9044, April 1998.

[19] E.P. Krider, R.C. Noggle, A.E. Pifer and D.L. Vance, "Lightning Direction-Finding Systems for Forest Fire Detection," Bulletin of the American Meteorological Society, vol. 61, no. 9, pp. 980-986, September 1980.

[20] W.R. Burrows, "Statistical Models for Lightning Prediction Using Canadian Lightning Detection Network Observations," 16th Conference on Probability and Statistics in the Atmospheric Sciences, American Meteorological Society, Orlando, Florida, January 13-172002.

[21] P.J. Lewis, "Winter Lightning in The Maritime Provinces of Canada," 16th International Lightning Detection Conference, Global Atmospherics, Inc., Tucson, Arizona, November 7-8, 2000. 
[22] Vaisala, "Vaisala Has Acquired Global Atmospherics Inc. Of USA," March 2002. [News Release]

http://www.lightningstorm.com/tux/jsp/about/news/acquisition.jsp

[23] Vaisala, "Vaisala STRIKEfax Fax Lightning Verification Report," 1998-2004. http://www.lightningstorm.com/tux/jsp/guide/products/analysis/strikefax/index.jsp

[24] M.J. Grogan, "Vaisala's U.S. National Lightning Detection Network: Report on the 2002-2003 U.S. NLDN System-wide Upgrade," June 2004. [Online Brochure] http://www.lightningstorm.com/jsp/discover/nldn/VN_20022003_NLDN_Upgrade_Article_June-2004.pdf

[25] R.E. Orville, G.R. Huffines, W.R. Burrows, R.L. Holle and K.L. Cummins, "The North American Lightning Detection Network (NALDN) - First Results: 1998-2000," Monthly Weather Review, vol. 130, no. 8, pp. 2098-2109, August 2002.

[26] K.L. Cummins, R.B. Pyle and G. Fournier, "An integrated North American Lightning Detection Network," Proceeding of the 11th International Conference on Atmospheric Electricity, Guntersville, AL, pp. 218-221, June 7-11, 1999.

[27] W.R. Burrows, "Verification of Statistical Models For Lightning Prediction," [Online Document] http://www.rap.ucar.edu/research/verification/pdf/burrows.pdf

[28] Global Atmospherics, Inc., "Global Atmospherics Building the New NLDN," Tucson, Arizona, February 2002. [News Release] http://www.lightningstorm.com/tux/jsp/about/news/nldn_upgrade.jsp

[29] Vaisala, "Environment Canada's CLDN: Canadian Lightning Detection Network," January 2004. [Online Brochure] http://www.lightningstorm.com/jsp/discover/nldn/CLDN_Brochure_B210413ENa_Jan2004.pdf

[30] R. Siikamaki, "Vaisala expands into lightning data services: Global Atmospherics Inc. joins Vaisala," June 2002. [News Release] http://www.vaisala.com/DynaGen_Attachments/Att18434/VN159_p04_05.pdf.pdf

[31] Vaisala, "Historic Analysis Products \& Services," 1998-2004 http://www.lightningstorm.com/tux/jsp/guide/products/analysis/index.jsp

[32] Vaisala, "Lightning Notification Products and Services," 1998-2004. http://www.lightningstorm.com/tux/jsp/guide/products/data/notification/index.jsp

[33] Environment Canada, "e-Weather Service Descriptions," April 2004. http://www.weatheroffice.pyr.ec.gc.ca/e-products/default_e.aspx 
[34] R. Bernstein, R. Samm, K. Cummins, R. Pyle and J. Tuel, "Lightning detection network averts damage and speeds restoration," IEEE Computer Applications in Power, vol. 9, no. 2, pp. 12-17, April 1996.

[35] K.L. Cummins, E.P. Krider and M.D. Malone, "The U.S. National Lightning Detection Network and Applications of Cloud-to-Ground Lightning Data by Electric Power Utilities," IEEE Transactions on Electromagnetic Compatibility, vol. 40, no. 4, pp. 465-480, November 1998.

[36] R.E. Orville, "Calibration of a magnetic direction finding network using measured triggered lightning return stroke peak currents," Journal of Geophysical Research, vol. 96, pp. 17135-17142, September 1991.

[37] V.P. Idone, A.B. Saljoughy, R.W. Henderson, P.K. Moore and R.B. Pyle, "A reexamination of the peak current calibration of the National Lightning Detection Network," Journal of Geophysical Research, vol. 98, pp. 18323-18332, 1993.

[38] Vaisala, "Vaisala STRIKEnet Lightning Verification Report," 1998-2004. http://www.lightningstorm.com/tux/jsp/guide/products/analysis/strikenet/index.jsp

[39] Vaisala, "STRIKEnet Report 866 - Sample ellipse map," May 2002. http://www.lightningstorm.com/htm/guide/products/analysis/strikenet/samples/ellipse_normal.htm

[40] K.L. Cummins, W.L. Hiscox, A.E. Pifer. and M.W. Maier, "Performance analysis of the U.S. National Lightning Detection Network," 9th International Conference on Atmospheric Electricity, International Commission on Atmospheric Electricity, St. Petersburg, Russia, June 15-19, 1992.

[41] Vaisala, "Lightning Verification Report Frequently Asked Questions,"1998-2004. http://www.lightningstorm.com/tux/jsp/cs/products/strikefax/faq/index.jsp

[42] M.W. Maier and M.B. Wilson, "Accuracy of the NLDN real-time data service at Cape Canaveral, Florida," International Lightning Detection Conference, Global Atmospherics, Inc., Tucson, Arizona, Nov. 6-8, 1996.

[43] V.P. Idone, D.A. Davis, P.K. Moore, Y. Wang, R.W. Henderson, M. Ries and P. F. Jamason, "Performance evaluation of the U.S. National Lightning Detection Network in eastern New York. Part II: Location accuracy," Journal of Geophysical Research, vol. 103, no. D8, pp. 9057-9069, April 1998.

[44] W. Janischewskyj, V. Shostak, A.M. Hussein and W. Chisholm, "Estimation of Lightning Location System Accuracy Using CN Tower Lightning Data," 23rd International Conference on Lightning Protection, Associazione Elettrotecnica ed Elettronica Italiana, Firenze, Italy, September 23-27, 1996 
[45] V.P. Idone, D.A. Davis, P.K. Moore, Y. Wang, R.W. Henderson, M. Ries and P. F. Jamason, "Performance evaluation of the U.S. National Lightning Detection Network in eastern New York. Part I: Detection efficiency," Journal of Geophysical Research, vol. 103, no. D8, pp. 9045-9055, April 1998.

[46] E.M. Thomson, M.A. Galib, M.A. Uman, W.H. Beasley and M.J. Master, "Some features of stroke occurrence in Florida lightning flashes," Journal of Geophysical Research, vol. 89, no. D3, pp. 4910-4916, 1984.

[47] N. Herodotou, W.A. Chisholm and W. Janischewskyj, "Distribution of lightning peak stroke currents in Ontario using an LLP system," IEEE transactions on Power delivery, vol. 8, no. 3, July 1993.

[48] A.M. Hussein, W. Janischewskyj, M. Milewski, V. Shostak, W. Chisholm and J.S. Chang, "Current Waveform Parameters of $\mathrm{CN}$ tower Lightning Return Strokes," Journal of Electrostatics, vol. 60, no. 2-4, pp. 149-162, March 2004.

[49] A.M. Hussein, W. Janischewskyj, M. Milewski, V. Shostak and J.S. Chang, "Waveform Parameters of Fields Generated by Lightning Strokes to the CN tower and to Objects in its Vicinity," 2003 IEEE Bologna Power Tech Conference Proceedings, vol. 4, Faculty of Engineering, University of Bologna, Bologna, Italy, June 23-26, 2003.

[50] V.A. Rakov, D.E. Crawford, K.J. Rambo, G.H. Schnetzer, M.A. Uman, and R. Thottappillil, "M-Component Mode of Charge Transfer to Ground in Lightning Discharges," Journal of Geophysical Research, vol. 106, no. D19, pp 22817 - 22831, 2001.

[51.] Personal communication from Chief Scientist Ken Cummins of Vaisala Inc.

[52] Personal communication from Research Engineer John Cramer and Chief Scientist Ken Cummins of Vaisala Inc.

[53] D. Dockendorff and K. Spring, "The Canadian Lightning Detection Network Novel Approaches for Performance Measurement and Network Management," 2005 World Meterological Orgainization Technical Conference on Meteorological and Environmental Instruments and Methods of Observation (TECO-2005), Bucharest, Romania, May 4-7, 2005. 


\section{Appendix A}

\section{Abbreviations}

$\begin{array}{ll}\text { ARSI } & \text { Atmospheric Research Systems, Inc. } \\ \text { BLM } & \text { Bureau of Land Management } \\ \text { CLDN } & \text { Canadian Lightning Detection Network } \\ \text { DE } & \text { Detection Efficiency } \\ \text { DF } & \text { Direction Finder (Magnetic) } \\ \text { EPRI } & \text { Electric Power Research Institute } \\ \text { GAI } & \text { Global Atmospherics, Inc. } \\ \text { GDS } & \text { GeoMet Data Services } \\ \text { GMT } & \text { Greenwich mean time } \\ \text { GPS } & \text { Global Positioning System } \\ \text { ICLRT } & \text { International Center for Lightning Research and Testing } \\ \text { IMPACT } & \text { IMProved Accuracy from Combined Technology } \\ \text { LF } & \text { Low Frequency (30 to 300 kHz) } \\ \text { LLP } & \text { Lightning Location and Protection, Inc. } \\ \text { LLS } & \text { Lightning Location System } \\ \text { LPATS } & \text { Lightning Positioning and Tracking System } \\ \text { MSC } & \text { Meteorological Service of Canada } \\ \text { NALDN } & \text { North American Lightning Detection Network } \\ \text { NCC } & \text { Network Control Center }\end{array}$


NLDN National Lightning Detection Network (U.S.)

NSSL National Severe Storm Laboratory (U.S.)

RF Radio Frequency

RNSS Range-normalized signal strength

SUNYA State University of New York at Albany

TLM Transmission line model

TOA Time-of-Arrival

UTC Coordinated universal time

VHF Very High Frequency (30 to $300 \mathrm{MHz}$ )

VLF Very Low Frequency ( 3 to $30 \mathrm{kHz}$ ) 